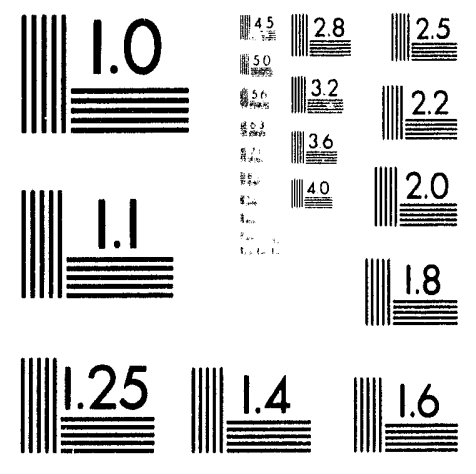



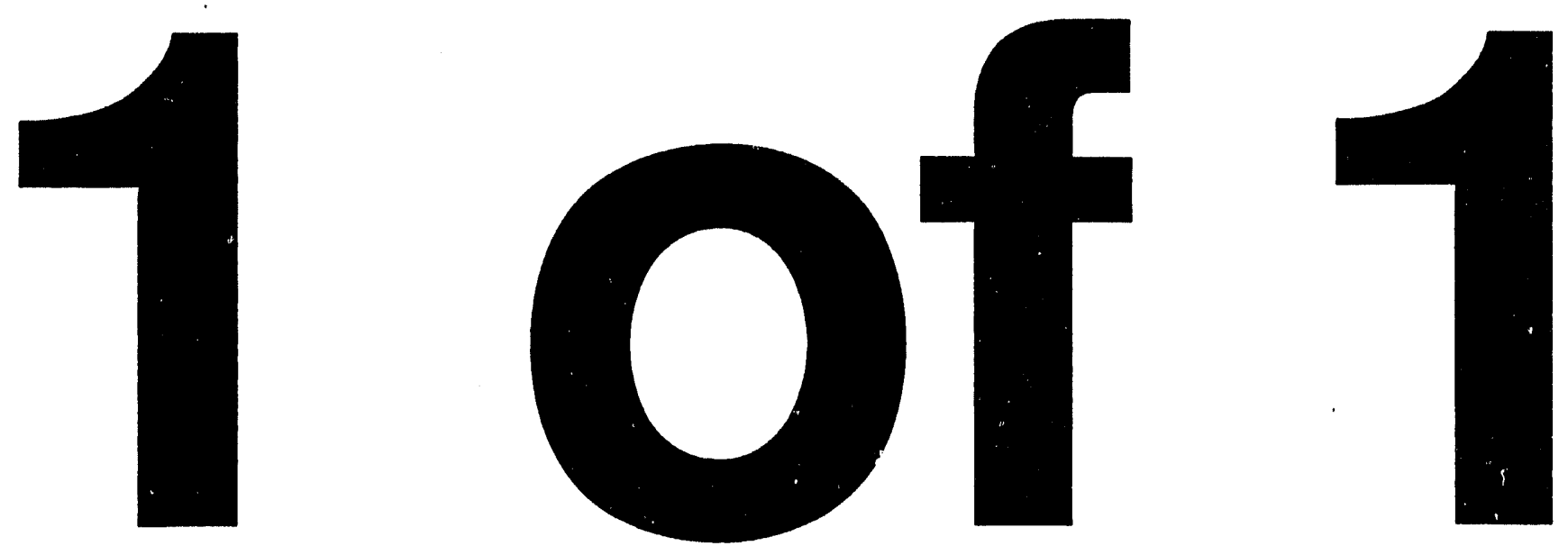


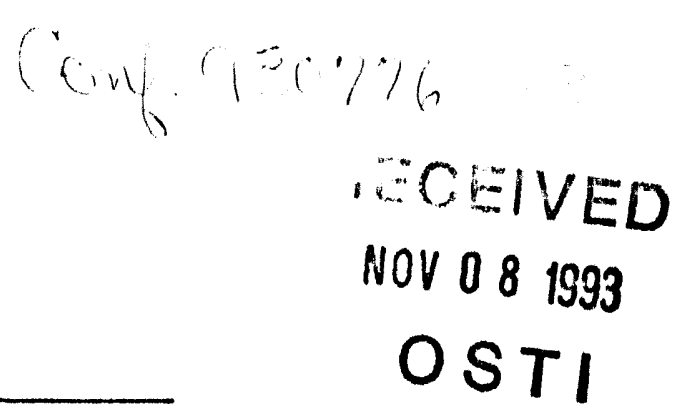

\title{
INTEGRAL EQUATIONS FOR THE MICROSTRUCTURES OF SUPERCRITICAL FLUIDS
}

\author{
Lloyd L. Lee*, and Henry D. Cochrant
}

*School of Chemical Engineering and Materials Science, University of Oklahoma, Norman, OK 73019

TChemical Technology Division, Oak Ridge National Laboratory, Oak Ridge, TN 37831

\section{Abstract}

Molecular interactions and molecular distributions are at the heart of the supercritical behavior of fluid mixtures. The distributions, i.e. structures, can be obtained through any of the thrie routes: (i) scattering experiments, (ii) Monte Carlo or molecular dynamics simulation, and (iii) integral equations that govern the relation between the molecular interactions $u(r)$ and the probability distributions $g_{i j}(r)$. Most integral equations are based on the Ornstein-Zernike relation connecting the total correlation to the direct correlation. The $\mathrm{OZ}$ relation requires a "closure" equation to be solvable. Thus the Percus-Yevick, hypernetted chain, and mean spherical approximations have been proposed.

We outline the numerical methods of solution for these integral equations, including the Picard, Labik-Gillan, and Baxter methods. Solution of these equations yields the solvent-solute, solvent-solvent, and solute-solute pair correlation functions (pcf's). Interestingly, these pcr's exhibit characteristical signatures for supercritical mixtures that are classified as "attractive" or "repulsive" in nature. Close to the critical locus, the pcf shows enhanced first neighbor peaks with concomitant long-range build-ups (sic attractive behavior) or reduced first peaks plus long-range depletion (sic repulsive behavior) of neighbors.

For ternary mixtures with entrainers, there are synergistic effects between solvent and cosolvent, or solute and cosolute. These are also detectable on the distribution function level.

The thermodynamic consequences are deciphered through the Kirkwood-Buff fluctuation integrals $\left(G_{i j}\right)$ and their matrix inverses: the direct correlation function integrals (DCFI's). These quantities connect the the correlation functions (microscopic quantities) to the chemical potential derivatives (macroscopic variables) thus acting as "bridges" between the two Weltanschauungen. As a consequence, the partial molar volumes, the isothermal compressibility, and the solubility can all be expressed in terms of the $G_{i j}$ and the DCFI's. Practical implications of these connections are that in selecting and designing extractive supereritical agents, one can predict the attractive or repulsive behavior, the appropriate solvent-solute pair, possible synergism, and P-T-x ranges that are operable for the given extraction.

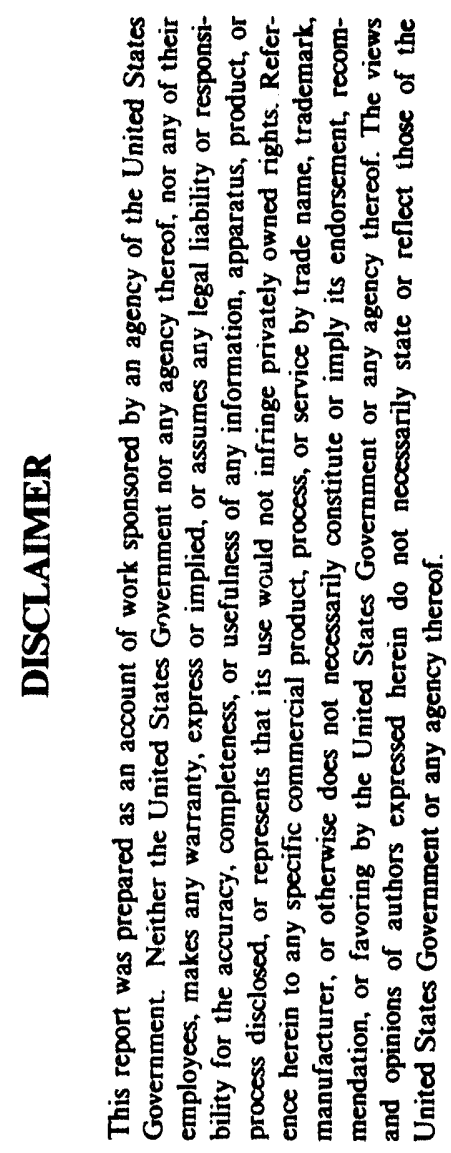

In addition, behavior of supercritical mixtures near an adsorbing wall can be analyzed by density profiles generated by the inhomogeneous Ornstein-Zemike type integral equations. This has significance on supercritical fluid chromatography.

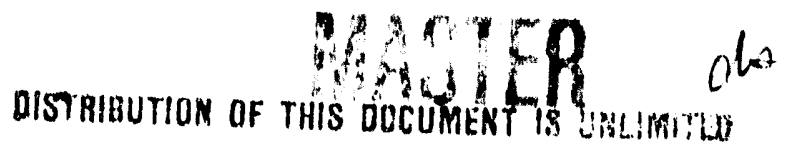


formulas; and (iii) these thermodynamic proparties are used to characterize the supercritical mixtures.

In the following, the sections are structured to first introduce the integral equations. The distribution functions are related to the thermodynamics through the Kirkwood-Buff solution theory. Then the numerical methods for solving the integral equations are outlined. These tochniques are applied to produce the solvent-solute and solute-solute pcf and their characteristics are ascertained for attractive and repulsive SCF. The thermodynamic properties are examined using these pef. We not only examine binary supercritical mixtures, but also ternary systems. We exhibit the conditions under which cosolvent or cosslute can have either cooperative effects, or interference with the solute. Due to the importance of supercritical fluid chromatography, we also lay the groundwork for a fundamental treatment of the molecular distributions of components of supercritical mixtures at aturactive and repulsive solid interfaces.

\section{Int egral Equation and Molecular Distributions}

We introduce first the terminology of statistical mechanics. For an N-body system, the Hamialtonian $H_{N}$ or the total energy of the system is

$$
H_{N}\left(\mathbf{p}^{N}, \mathbf{r}^{N}\right)=\sum_{i=1}^{N} \frac{p_{i}^{2}}{2 m}+V_{N}\left(\mathbf{r}_{1} \ldots, \mathbf{r}_{N}\right)
$$

The classical canonical partition function is then

$$
Z_{N}=\frac{Q_{N}}{N ! \Lambda^{3 N}}
$$

where the configurational integral, $Q_{N}$, is defined as

$$
Q_{N} \equiv \int d \mathbf{r}_{1} \cdots d \mathbf{r}_{N} \exp \left[-\beta V_{N}^{\prime}\left(\mathbf{r}_{1}, \ldots, \mathbf{r}_{N}\right)\right]
$$

In terms of the configurational integral, the two-body density function or the pair density is given by

$$
\rho^{(2)}\left(\mathbf{r}_{1}, \mathbf{r}_{2}\right)=\frac{N(N-1)}{Q_{N}} \int d \mathbf{r}_{3} \cdots d \mathbf{r}_{N} \exp \left[-\beta V_{N}\left(\mathbf{r}_{1}, \ldots, \mathbf{r}_{N}\right)\right]
$$

The pair correlation function is then

$$
g\left(r_{12}\right) \equiv \frac{\rho^{(2)}\left(r_{12}\right)}{\rho^{2}}
$$

In addition, the total correlation function $h(r)$ is

$$
h\left(r_{12}\right) \equiv g\left(r_{12}\right)-1
$$

The direct correlation function $c(r)$ is defined in terms of the Ornstein-Zemike relation

DEFINITION: The Ornstein-Zernike Relation. The direct corrclation function, $c\left(\mathbf{r}_{1}, \mathbf{r}_{2}\right)$, is defined in 
terms of the total correlation function $h\left(\mathbf{r}_{1}, \mathbf{r}_{2}\right)$ through a convolution integral

$$
h\left(\mathbf{r}_{1}, \mathbf{r}_{2}\right)-c\left(\mathbf{r}_{1}, \mathbf{r}_{2}\right)=\rho \int d \mathbf{r}_{3} h\left(\mathbf{r}_{1}, \mathbf{r}_{3}\right) c\left(\mathbf{r}_{3}, \mathbf{r}_{2}\right)
$$

Knowing $h\left(r_{1}, r_{2}\right)$ one can obtain the direct correlation function. In bipolar coordinates, the $\mathrm{OZ}$ equation assumes the form

$$
h(r)-c(r)=\frac{2 \pi \rho}{r} \int_{0}^{\infty} d s s c(s) \int_{\mid r=1}^{r+\infty} d t \operatorname{th}(t)
$$

Many integral equation theories for correlation functions are based on the $\mathrm{OZ}$ relation. Since the $\mathrm{OZ}$ defines the dcf $c(r)$, it requires a second equation for unique solution of both $c(r)$ and $h(r)$. This second equation is called the closure relation. The closure is normally based on cluster diagrams (e.g., the hypernetted chain equation), functional expansions of a generating functional, or microscopic force balances. The "exact" closure is given in terms of a bridge function $B(r)$, i.e.,

$$
g(r)=\exp [-\beta u(r)+h(r)-c(r)+B(r)]
$$

$B(r)$ has well-defined, though difficult to evaluate, cluster diagrams. When $B(r)$ is set (arbitrarily) to zero, we have the approximate HNC closure.

$$
g(r) \stackrel{H N C}{=} \exp [-\beta u(r)+h(r)-c(r)]
$$

For PY (another approximate closure),

$$
B(r)=\ln (g(r)-c(r))-h(r)+c(r)
$$

Thus

$$
c(r) \stackrel{P Y}{\approx} g(r)-g(r) \exp [\beta u(r)]
$$

\section{Distribution Functions and Thermodynamic Properties}

The distribution functions are related to thermodynamic quantities through the well-known energy, virial pressure, and isothermal compressibility $\left(K_{T}\right)$ equations. However, for the study of SCF, we are interested in the solubility, the partial molar volume (PMV), and $K_{T}$. To oblain these first two variables, we choose another route: the Kirkwood-Buff $(\mathrm{KB})$ factors, $G_{\text {ap, }}$ defined as

$$
G_{\alpha \beta}=G_{\beta \alpha}=\tilde{0} d r\left\{\left[g_{\alpha \beta}(r)-1\right] 4 \pi r^{2}\right\}=\int d r h_{\alpha \beta}(r)
$$

\subsection{THE KIRKWUOD-BUFF THEORY OF SOLUTIONS}

We first define the Jacobian A of the derivatives of the chemical potentials $\mu_{i}$ with respect to mole 
numbers $N_{j}$, and its matrix inverse $\mathbf{B}$ :

\section{$A \cdot B=I$}

where

$$
\begin{aligned}
& A_{\alpha \beta} \equiv \frac{V}{k T}\left[\frac{\partial \mu_{\alpha}}{\partial\left\langle N_{\beta}\right\rangle}\right]_{T, V, N_{\gamma \neq \beta}}=\frac{1}{k T}\left[\frac{\partial \mu_{\alpha}}{\partial \rho_{\beta}}\right]_{T, \rho_{\gamma+\beta}} . \\
& B_{\alpha \beta} \equiv \frac{k T}{V}\left[\frac{\partial\left\langle N_{\alpha}>\right.}{\partial \mu_{\beta}}\right]_{T, V \mu_{\gamma, \beta}}=k T\left[\frac{\partial \rho_{\alpha}}{\partial \mu_{\beta}}\right]_{T_{\mu,+\beta}}=\rho_{\alpha} \rho_{\beta} G_{\alpha \beta}+\rho_{\alpha} \delta_{\alpha \beta,}
\end{aligned}
$$

$I$ is the unit diagonal matrix, and $\delta_{\alpha \beta}$ is the Kronecker delta. We note that the B matrix is related to the number fluctuations in terms of $G_{i j}$ (thus also the name fuctuation integrals).

Kirkwood and Buff have shown that a number of thermodynamic quartities are related to these fluctuation integrals: namely, partial molar volume, isothermal compressibility, and solubility. The partial molar volume is given by (in a binary mixture of solvent 1 and solute 2)

$$
\rho \bar{V}_{2}=\frac{1+\rho x_{1}\left(G_{11}-G_{12}\right)}{1+\rho x_{1} x_{2} \Delta}
$$

and the isothermal compressibility of the mixture

$$
\rho k T K_{T}=\frac{1+\rho x_{1} G_{11}+\rho x_{2} G_{22}+\rho^{2} x_{1} x_{2}\left(G_{11} G_{22}-G_{12}^{2}\right)}{1+\rho x_{1} x_{2} \Delta}
$$

where

$$
\Delta=G_{11}+G_{22}-2 G_{12}
$$

For a binary solution of solute 2 at infinite dilution in solvent $1, x_{2} \rightarrow 0$, Equations (8) and (9) simplify to

$$
\rho_{1}^{0} \bar{V}_{2}^{*}=1+\rho_{1}^{0}\left(G_{11}^{0}-G_{12}\right)
$$

and

$$
\rho_{i}^{0} k T K_{T}^{0}=1+\rho_{1}^{0} G_{11}^{0} .
$$

It is also possible to express the PMV and $K_{T}^{0}$ (i.e., the isothermal compressibility of pure solvent) in terms of the direct correlation function integrals (DCFI)

$$
\rho_{1}^{0} \bar{V}_{2}^{-}=\rho_{1}^{0} k T K_{T}^{0}\left(1-C_{12}^{m}\right)
$$

and

$$
\rho_{1}^{0} k T K_{T}^{0}=\left(1-C_{11}^{0}\right)^{-1}
$$

In applications, it is convenient to define the Krichevskii parameter $\delta_{2}$

$$
\delta_{2} \equiv \lim _{N_{2} \rightarrow 0}\left[N \frac{\partial P}{\partial N_{2}}\right]_{T, V, N_{k} * 2}
$$




$$
=\rho_{1}^{0} k T \bar{V}_{2}^{-}\left(K_{T}\right)^{-1}=p_{1}^{0} k T\left(1-C_{12}\right)
$$

It will also be convenient to use the dimensionless Krichevskii parameter $\delta_{2}{ }^{*}$ defined by

$$
\delta_{2}^{*}=\frac{\delta_{2}}{p_{1}^{0} k T}
$$

The solubility of solute 2 in the supercritical fluid can be expressed in terms of the enhancement factor E

$$
y_{2}=E \frac{P}{P}
$$

The enhancement factor expresses the actual solubility over its ideal gas value. It is given in terms of the fugacity coefficient $\phi_{2}$ of the vapor phase as

$$
\left.E=\phi_{2}^{\text {solid }} \operatorname{expl} \int d P \beta v_{2}^{\text {sold }}\right] / \phi_{2}
$$

From thermodynamic relations, we know

$$
\begin{aligned}
\ln \phi_{2} & =\int_{0}^{P} \overline{d P}\left[\frac{\bar{V}_{2}}{k T}-\frac{1}{\bar{P}}\right] \\
& =\int_{0}^{P} \overline{d P}\left[K_{T}\left(1-C_{\overline{12}}\right)-\frac{1}{\bar{P}}\right]
\end{aligned}
$$

Or in terms of an integral over density

$$
\ln \phi_{2}=-\int_{0}^{p} \frac{d \bar{\rho}}{\bar{\rho}} C_{\tilde{12}}-\ln Z^{0}
$$

The configurational chemical potential $\mu_{2}^{\prime m}$ is accordingly

$$
\beta \mu_{2}^{\prime *}=-\int_{0}^{p} \frac{d \bar{p}}{\bar{\rho}} C_{\ddot{12}}
$$

Since $\phi_{2}$ determines the solubility (or enhancement), it is interesting to see that according to the pressure integral (eq.18), the enhancement is due to the divergence of the isothermal compressibility $K_{T}$ (with a sign determined by $1-C_{12}$ ). But according to the density integral (eq,19), $\phi_{2}$ is determined by the "short-ranged" integrand $C_{\overline{12}}^{-}$only! This is causing some current controversy on whether the solubility anomaly is due to "long" or "short" range correlations.

It is appropriate here to clarify the semantics used in literature for SCF. A thermodynamic quantity can be "finite" or "divergent" in the albeit narrow sense that it is free of or scaled by the isothermal compressibility $K_{T}$, which of course diverges at the solvent CP. For example, eq.(3.10) says that the PMV is a "divergent" quantity for SCF, since it scales as $K_{T}$. The chemical potential eq.(3.18) is "finite", since it does not scale with $K_{T}$. A quantity is either "short" or "long" ranged depending on whether it involves only short-ranged correlation functions (e.g., $c_{12}(r)$ ) or it involves long-ranged correlation functions $\left(\right.$ e.g., $\left.h_{12}(r)\right)$. Thus the $\mathrm{KB}$ factors $G_{12}$ are long-ranged, while the DCFI are 
short-ranged quantities. Thirdly, the meaning of "local" vs. "global" quantities refers to the quantities that are obtained by considering only the radial distance from one to two (or few) molecular diameters (local), vs many (e.g., 20 to 50, etc.) molecular diameters (global). Thus the local density enhancement (LDE) is a "local" quantity, because it is defined within 1 to 3 diameters of the solvent molecule. But since the LDE involves the long-ranged $h_{12}(r)$, it is actually, in our parlance, a "long-ranged" quantity (or better yet, quantity depending on long-ranged correlation functions). LDE is "finite", because it is local and not scaled with $K_{T}$.

In light of our "new" definitions, what can we say about the solubility enhancement near solvent $\mathrm{CP}$, a prototypical supercritical behavior? We shall view this solubility question as a "solubility variation" question, namely, how fast the solubility changes with respect to either pressure variations or temperature variations? because what is interesting in supercritical technology and extraction-separation is the "rapid" change of solubilities with pressure, or temperature, when going across the $\mathrm{CP}$. The the derivative of fugacity with respect to pressure, according to $(3,16)$ is

$$
\frac{\partial \ln \phi_{2}}{\partial P}=K_{T}\left(1-C_{12}\right)-\frac{1}{P}
$$

Or, the solubility variation is

$$
\frac{\partial y_{2}}{\partial P}=-K_{T}\left(1-C_{12}\right)=-K_{T} \delta_{2} \cdot
$$

(Note that the same result is obtained from eq.(3.17)). Thus the solubility change is driven by $K_{T}$, a "divergent" quantity.

\subsection{ATTRACTIVE, REPULSIVE \& WEAKLY ATTRACTIVE MIXTURES}

The affinity of solvent molecules for a solute nucleus (solvation or densification) is expressed by the excess number, $N_{1}^{e x}$

$$
N_{1}^{a x} \equiv \lim _{N_{2} \rightarrow 0} \rho_{1}^{0} \int d r h_{12}(r)=\rho_{1}^{0} G_{12}
$$

This quantity can be related by matrix inverse to DCFI as

$$
N_{1}^{e x} \equiv \rho_{1}^{0} k T K_{T} C_{12}
$$

Synthesizing these relations, we oblain for the partial molar volume and the excess number

$$
\begin{aligned}
& \rho_{1}^{0} \bar{V}_{2}^{*}=\rho_{1}^{0} k T K_{T}-N_{1}^{e x}=K_{T} \delta_{2}=\rho_{1}^{0} k T K_{T} \delta_{2}^{*} \\
& N_{1}^{e x}=K_{T}\left(\rho_{1}^{0} k T-\delta_{2}\right)=\rho_{1}^{0} k T K_{T}\left(1-\delta_{2}^{*}\right)
\end{aligned}
$$

By splitting the total correlation $h_{12}(r)=c_{12}(r)+\gamma_{12}(r)$, where $\gamma_{12}(r)$ is the indirect correlation function, Chialvo and Cummings have recently shown that the PMV can also entertain a "finite" contribution and a "divergent" contribution:

$$
\begin{aligned}
\rho_{1}^{0} \bar{V}_{2}^{-} & =\rho_{1}^{0} \bar{V}_{2}^{- \text {fin }}+\rho_{1}^{0} \bar{V}_{2}^{- \text {divg }}= \\
& =\left(1+C_{11}^{0}-C_{12}^{\infty}\right)+\rho_{1}^{0} k T K_{T}\left(C_{11}^{0}-C_{12}\right)
\end{aligned}
$$


This is helpful in elucidating the solute-induced local effects when a solute molecule is introduced into the predominantly solvent environment. Equations $(3.23,24)$ entail the definitions of three categories of supercritical fluids: (i) attractive, (ii) repulsive, and (i) weakly attractive mixtures.

For negative Krichevskii parameter: $\delta_{2}<0$, eq.(3.23) says that $\bar{\nabla}_{2}^{-} \rightarrow-\infty$ as the CP of the solvent is approached, while eq. (3.24) says that the excess number $N_{1}^{e x} \rightarrow+\infty$. This is typically attractive behavior: volumetric collapse upon solute addition, and large excess number buildup.

By the same reasoning, we can define the other two categories. The table below summarizes the parameter ranges.

Table 3.1 Auractive, Repulsive and weakly Attractive Mixtures

\begin{tabular}{l|ccc}
\hline \hline Category & $\delta_{2}$ & $\lim \bar{V}_{2}^{\infty}$ & $\lim N_{1}^{\sigma^{2}}$ \\
\hline Attractive & $0>\delta_{2}^{\circ}$ & $\rightarrow-\infty$ & $\rightarrow+\infty$ \\
Weakly Attractive & $1>\delta_{2}^{\circ}>0$ & $\rightarrow+\infty$ & $\rightarrow+\infty$ \\
Repulsive & $\delta_{2}^{\circ}>1$ & $\rightarrow+\infty$ & $\rightarrow-\infty$ \\
\hline
\end{tabular}

\section{Numerical Solution of Integral Equations}

We use the PY equation as an example for discussing the numerical techniques of solving integral equations. In bipolar coordinates, the PY equation assumes the form

$$
y(r)=1+\frac{2 \pi \rho}{r} \int_{0}^{\infty} d s s C(s) \int_{|r \rightarrow|}^{r+\infty} d t \operatorname{th}(t)
$$

Eq. (4.1) coupled with the PY assumption

$$
C(r)=h(r)-y(r)+1
$$

forms a complete set of equations sufficient for the determination of the radial distribution function (rdf). For simple potentials (e.g., the hard-sphere potential), one can solve (3.1) by Laplace transforms. An analytical solution is obtained. For more complicated potentials, numerical solutions must be sought. A number of schemes have been developed. We shall introduce four such methods below.

\subsection{PICARD'S METHOD}

Picard's method refers to the solution by iterations. An initial guess of the solution function is substituted into the RHS of equation. An output function is calculated for the LHS. Next this output is substituted into the RHS as a new input to yicld a second output. The process is repeated until certain convergence criteria are satisfied. For PY, the convolution integral is evaluated in two parts:

$$
y(r)=1+\frac{2 \pi \rho}{r} \int_{0}^{\infty} d s s C(s)[E(r+s)-E(|r-s|)]
$$

and 


$$
E(x)=\int_{0}^{x} d t \ln (t)
$$

The direct and total correlation functions are related by

$$
C(r)=h(r)-y(r)+1
$$

The pair potential is known in advance. It enters (3.6) via the definition $h(r)=y(r) \exp [-\beta u(r)]-1$. The algorithm of solution commences with an initial guess $h^{[0]}(r)$, iterating through equations (3.4-6) until the so-called Neumann sequence $h^{(0)}, h^{(1)}, h^{[2]}, \ldots, h^{(n)} \ldots$ converges to a fixed point within prescribed limits. The steps are:

1. Fix the pair potential $u(r)$, temperature $T$, and density $\rho$.

2. Choose initial guess $h^{(0)}(r)$. The initial choice is important in the present method for numerical convergence, especially at high densities. For dilute gas, a good guess is

$$
g^{(0)}(r)=e^{-n(r) k t}
$$

For dense fluids, there is no sure guidance. In practice, one builds up the densities from low to high, using the previous rdf as input for the next higher density.

3. Obtain $E(x)$ from (3.5). Here one can construct a table of $E$ values at different $x$ for a given $h(r)$, and store them in memory for later use. One may use any standard quadratures for numerical integration (or the usual trapezoidal and Simpson's methods).

$$
E^{[0]}(x)=\int_{0}^{x} d t \operatorname{th}^{[0]}(t)
$$

$C(r)$ is oblained from

$$
C^{[0]}(r)=y^{[0]}(r)\left[e^{\mu(r) k T}-1\right]
$$

where $y^{(0)}=g^{(0)} \exp (u / k T)$

4. Obtain $y(r)$ from (3.4):

$$
y^{(1)}(r)=1+\frac{2 \pi p}{r} \int_{0}^{b} d s s C^{[0]}(s)\left[E^{[0]}(r+s)-E^{[0]}(|r-s|)\right]
$$

Since we cannot go to infinity numerically, the upper limit $b$ is chosen sufficiently large so that $C(b)$ is very small $\left(\equiv 10^{-7}\right)$. Normally, $b=60-100$ is adequate for $\mathrm{LJ}$ molecules and 200 for Coulomb electrostatic forces. ( $\sigma$ correspunds to the molecular size).

5. To guarantee convergence, $\operatorname{mix} y^{(1)}$ with $y^{(0)}$ before the the next iteration according to

$$
y_{\operatorname{mix}}^{(1)}(r)=\alpha y^{(0)}(r)+(1-\alpha) y^{(1)}(r)
$$


where $0<\alpha<1$ is the mixing parameter. In numerical analysis, this is called relaxation. For low density states, $\alpha$ is small (say, 0.4 ); for high density states, $\alpha$ is large (e.g., $\alpha=0.9$ for $\rho^{*}=0.7$ ). It is used to insure numerical stability. The judicious choice could only be made from experience in working with the equations.

6. This $y_{\mathrm{mix}}^{(1)}$ is used as input in (3.8) and (3.9) to get $E^{[1)}$ and $C^{(1)}$ (note that $g^{(1)}(r)$ $\left.=y_{\text {mid }}^{\prime \prime \prime}(r) \exp [-u(r) / k T]\right)$. Steps (iv) to (vi) are repeated. A sequence $y^{(0)}, y^{(1)}, y^{(2)} \ldots, y^{(k)}, \ldots$ is gencrated. If the Neumann sequence is convergent, we shall have the solution $y(r)$. Numerically, we impose the Cauchy condition that if

$$
\left|y^{|n+1|}(r)-y^{(n)}(r)\right|<\delta \quad \text { for all } r
$$

where $\delta$ is a small number (e.g., $\delta=0.0001$ ), we consider the sequence convergent and the solution $y(r)$ is set to $y^{[n+1]}(r)$. Other correlation functions are obtained from

$$
g(r)=y(r) e^{-\mu(r) k T}
$$

and

$$
C(r)=g(r)-y(r)
$$

\subsection{GILLAN'S METHOD}

Gillan's method consists essentially in dividing the pair correlation function into two parts, a coarse part and a fine part, then applying the Newton-Raphson (NR) method to speed up convergence. It has recently been refined by Labik et al. [3] and applied to the reference interaction site model by Monson [4] and Enciso [5] for polyatomic molecules. A remarkable feature of Gillan's method is its insensitivity to initial guesses for the numerical solution, a step critical in earlier methods. In addition, for most state conditions, the convergence is fast. These advantages have made this method the prime choice in solution of integral equations.

Earlier, Watts [6] has used a Newton-Raphson procedure on discretized integral equations. Gillan divided the pcf in two parts: one slowly varying, the other small and rapidly oscillating. The outline of the method is given below. A computer program is also provided in the Appendices.

The iterative procedure is based on the indirect correlation function (icf) $\mathcal{X} r$ ) defined by

$$
X(r) \div h(r)-C(r)
$$

(Note that the total correlation $h(r)$ is the sum, $h(r)=C(r)+\gamma(r)$, of the direct correlation $C(r)$ and the indirect correlation $\gamma(r)$ )). The $\mathrm{OZ}$ relation in the Fourier space could be written as

$$
\tilde{\gamma}(k)=\frac{\rho C^{2}(k)^{2}}{1-\rho \tilde{C}(k)}
$$

where tilde indicates Fourier transforms. In the new method the icf is divided into

$$
\gamma(r)=\gamma(r)+\Delta \gamma(r)
$$

or in discretized form 


$$
\gamma_{i}=\gamma_{i}^{k}+\Delta \gamma_{i}
$$

where $\gamma_{i}=\gamma(r=i \delta r), i=0,1,2,3, \ldots, N$, and $\delta r$ is the discretized grid size. $\gamma$ is the coarse part of the icf to be defined below, and $\Delta \gamma_{i}$ is the fine part. The coarse part is expressed as an expanision (spectral decomposition) in terms of a sel of orthogonal basis functions $p a \alpha=1,2,3, \ldots$ with expansion coefficients $a_{\mathrm{a}}$

$$
\gamma_{i}^{c}=\sum_{\alpha} a_{\alpha} P_{i}^{\alpha}
$$

Thus

$$
\gamma_{i}=\sum_{a} a_{a} P_{i}^{\alpha}+\Delta \gamma_{i}
$$

In addition

$$
\sum_{i} P_{i}^{\alpha} \Delta \gamma_{i}=0, \quad V \alpha
$$

In order to close the simultaneous set of equations, we need one additional relation between the icf and the dcf. This is furnished by the usual integral equations. For example in the PY closure

$$
C(r)=[1+\gamma(r)] f(r)
$$

or in HNC

$$
C(r)=e^{-\beta w(r)+x(r)}-\gamma(r)-1
$$

For arbitrarily chosen initial guess $\gamma_{i}$, eqs. (3.23 and 29) might not be satisfied simultaneously. Thus an iterative procedure commonly used in solving integral equations will be implemented. The procedure is repeated until certain convergence criteria are satisfied. The iterations are a combination of the Newton-Raphson steps (on the coarse part $\gamma_{i}{ }^{c}$ ) and Picard steps (on the fine part $\Delta \gamma_{i}$ ). Each combination (Newton-Raphson + Picard steps) is called a refinement cycle. The final solution will be denoted by asterisks: its expansion coefficients are $\left(a^{*}, a^{*}, \ldots\right)$, and the fine part is $\Delta \gamma_{i}^{*}$.

The coarse function subspace is spanned by a small number $v=10$ of basis functions $P^{\alpha}$, (these functions will be specified later). Initially, we choose arbitrary but reasonable $\gamma(r)$ and $\left(a_{\alpha}\right)$. If the choice of $\left(a_{1}, a_{2}, \ldots\right)$ is the proper set, we have the final solution. Substitution into (3.23) and (3.29), PY, for example, should give the same $X(r)$. Any difference indicates improper answer, A new set $\left(a_{1}, a_{2}, \ldots\right\}$ is to be produced via a Newton-Raphson procedure. The process is repeated until convergence is achieved. Suppose that the output derived through (3.23 and 29) from the input of an initial $\gamma_{i}$ is

$$
\gamma_{i}=\sum_{\alpha} a_{\alpha}^{\prime} P_{i}^{\alpha}+\Delta \gamma_{i}
$$

If $\gamma$ is the exact solution, we should have

$$
d_{\alpha} \equiv a_{\alpha}-a_{a}^{\prime}=0
$$


and

$$
\Delta \gamma_{i}=\Delta \gamma_{1}
$$

In most cases, first few iterations will not satisfy the above two conditions. To procard, we fix the $\Delta \gamma_{i}$ arbitrarily at the initial values and generate new expansion coefficients $a_{a}$ until they satisfy (3.32). These new $a$ 's are obtained by a Newton-Raphson formula

$$
\bar{\sigma}_{\alpha}=a_{\alpha}-\sum_{\beta}\left(J^{-1}\right)_{\alpha \beta} d_{\beta}
$$

where $\mathrm{J}$ is the Jacobian

$$
J_{\alpha \beta} \equiv \frac{\partial d_{\alpha}}{\partial a_{\beta}}
$$

evaluated at the old estimates $\left(a_{a}\right)$. The derivatives are taken at constant $a_{x-\beta}$ and constant $\Delta y_{i}$. These corrective iterations on the coarse part are called NR cycles. The cycles are continued until the new $a$ 's do not change from their previous values (Cauchy condition). Let the primed quantities represent outputs from an NR cycle, we have for the final $a * ' s$

$$
\gamma^{*}=\sum_{a} a_{a}^{*} P_{i}^{a}+\Delta \gamma_{i}
$$

compared with thi: previous input

$$
\gamma_{i}^{*}=\sum_{\alpha} a_{\alpha}^{*} P_{i}^{\alpha}+\Delta \gamma_{i}
$$

Note that the expansion coefficients stay the same after the NR cycle while the fine part $\Delta \gamma$ changes. These $a$ *'s are consistent with the input $\Delta \gamma$. However, these $\Delta \gamma$ do not yet satisfy (3.33). To obtain the final solution we carry out Picard iterations on $\Delta \gamma_{i}$ i.e. the output $\Delta \gamma_{i}$ is used as the new input for the next NR cycle on $a$ 's. The entire procedure looks like this

Series of refinement cycles $=[$ fixed $\Delta \gamma] \rightarrow N R$ Cycles on $a^{\prime} s \rightarrow[$ new $\Delta \gamma] \rightarrow N R$ Cycles on $a^{\prime} s \rightarrow\left[\right.$ new $\left.\Delta \gamma^{\prime}\right] \rightarrow \ldots$

The cycles continue until condition (3.33) is satisfied to with a small tolcrance. After a number of cycles (in practice, 6 to 7 refinements), the final solution is obtained.

Labik et al. [7] used sine functions instead of the roof functions. They obtained threefold to ninefold faster convergence than the Gillan scheme. This was made possible in part by avoiding evaluating the Fourier transforms in the NR cycles.

\subsection{LABIK'S METHOD}

Labik et al. proposed to use the Fourier series as the basis functions instead of the roof function of Gil. lan. Since in solution of integral equations, one iterates between the closure relation: $c(r)=f(h(r))$, and the OZ relation: $\bar{h}(k)=F(C(k))$, two Fourier transformations are needed for one iteration. (This is what Gillan's method does). Despite the fast Fourier algorithm, it still saves considerable computer time if 
this could be avoided. Labik used first order Taylor's expansion of the closure relation:

$$
Q(r) \approx Q^{0}(r)+\phi^{0}(r)\left[\Gamma(r)-\Gamma^{0}(r)\right]
$$

where $\phi^{0}(r)$ is the derivative $d Q / d \Gamma$ at $\Gamma^{0}, Q(r) \equiv r c(r)$, and $\Gamma(r) \equiv r \gamma(r)$. This derivative could be based on the PY closure or the HNC (or any other) closure. The equation is Fourier transformed to give

$$
\tilde{Q}(j)=\tilde{Q}^{0}(j)+\tilde{\psi}(j, k)\left[\tilde{\Gamma}(k)-\tilde{\Gamma}^{0}(k)\right]
$$

The coefficient matrix $\dot{\psi}$ is given by

$$
\bar{\psi}_{j k}=\frac{1}{N} \sum_{i=1}^{N-1} \phi_{i}^{0}[\cos (\pi i(k-j) / N)-\cos (\pi i(k+j) / N)]
$$

Thus in the NR cycle, there is no need to transform back to $r$-space. Note that the $\mathrm{OZ}$ relation holds in the Fourier space:

$$
\tilde{\Gamma}(k)=\frac{\rho \mathscr{Q}(k)^{2}}{k-\rho k \bar{Q}(k)}
$$

\subsection{BAXTER'S METHOD}

The Labik-Gillan method requires the discretization of $\gamma_{i j}(r)$ and $c_{i j}(r)$ into a set of $\mathrm{N}$ values representing $h_{i j}\left(r_{i}\right)$ and $c_{i j}\left(r_{i}\right)$, where $r_{i}=i \Delta r$ and $\mathrm{i}=1, \ldots, \mathrm{N}$. The Labik-Gillan method also requires the assumption $h_{i j}(r)=0$ for $r \geq R=N \Delta r$. A similar truncation is not always necessary for $c_{i j}(r)$, i.e., when using PY or MSA closures with finite-ranged potentials. >From the comparison by Cummings and Monson between numerical and analytical solutions of $\mathrm{OZ}$ equations (31), it is clear that any numerical scheme which requires that $h_{i j}(r)=0$ for $r>R$ is unable to approach the CP closely. This suggests that a method not dependent on $r$-space truncation of $h_{i j}(r)$ should be employed in the vicinity of the C.P.

Based on factorization of the $\mathrm{OZ}$ equation for a single component, Baxter (32) proposed a numerical algorithm for the calculation of $h(r)$ under the sole assumption that $c(r)$ is finite in range. He showed that if $c(r)=0$ for $r>R$ (i.e., the direct correlation function is finite in range, as it is in the PY and MSA approximations for finite potentials) then the $\mathrm{OZ}$ equation can be factored into two equations.

$$
\begin{aligned}
& r h(r)=-q^{\prime}(r)+2 \pi \rho \int_{0}^{R} d t q(t)(r-t) h(|r-t|) \\
& r c(r)=-q^{\prime}(r)+2 \pi \rho \int_{r}^{R} d t q^{\prime}(t) q(t-r)
\end{aligned}
$$

where

$$
q(r)=0 \text { for } r<0 \text { and } r \geq R
$$

Baxter suggested that the two equations (8) and (9) could be used, in conjunction with a closure relation, 10 iterate on the function $q(r)$, thus yielding a numerical method for solving the $\mathrm{OZ}$ equation where the solution of $h(r)$ at $r \geq R$ is not required. 
An original version of such an algorithm for the single component case was derived by Baxter (33), employing a single integral equation which coupled equations (8) and (9). It was solved by Watts (34) with the PY closure using the NR method with grid $0.05 \sigma$ and with the potential cutoff varied from $3.5 \sigma$ to $6.0 \sigma$. To approach the $\mathrm{CF}$, a series of runs with various densities around different isothermis was required.

Recently a variant of this method was introduced by Cummings and Monson (31) which turns equations (8) and (9) into a DI scheme for function $q(r)$.

They indicated that their DI method had the following difficulties: Small grids $\Delta r=0.005$ were required; so, a large number of points, $\mathrm{N}=300$, had to be used. When $k T / \varepsilon<1.4$, the DI scheme encountered convergence problems. They also coupled equations (8) and (9) and solved using the NR technique. When they applied the NR algorithm, it was computationally intensive when $\mathrm{N}>\mathrm{O}\left(10^{2}\right)$, and a good initial guess was necessary to ensure convergence. When we tried to extend this method to typical, binary supercritical solutions, very dissimilar molecules were encountered, and the above problems became worse. When the solute molecule has large size $\left(\sigma_{11}<<\sigma_{22}\right)$, we needed a large number of points $\mathrm{N}$ to cover the solute potential. Direct application of the NR method became harder because of the need to handle four times as many variables and a larger matrix to solve the simultancous equations. For typical supercritical solutions the solute molecules have large $\varepsilon_{22}$ values compared to the solvent $\varepsilon_{11}$. When we reach the solvent $C P, k T / \varepsilon_{11}$ is approximately 1.3 ; so, we encounter very low reduced temperatures with respect to solute molecules $\left(k T / \varepsilon_{22}<1\right)$. These difficulties led us to seek a more delicate algorithm to solve the $\mathrm{OZ}$ equations for binary solutions near the solvent $\mathrm{CP}$.

\subsubsection{METHOD OF CALCULATION}

Baxter reformulated the $\mathrm{OZ}$ equations of mixtures into two sets of equations given by

$$
r c_{i j}(|r|)=-q_{i j}^{\prime}(r)+2 \pi \sum_{k} \rho_{k} \int_{S_{k j}}^{R_{m}} d t q_{k i}(t) q_{k j}^{\prime}(r+t)
$$

for $R_{i j} \geq r \geq S_{i j}$ and

$$
r h_{i j}(|r|)=-q^{\prime}{ }_{i j}(r)+2 \pi \sum_{k} \rho_{k} \int_{S_{i k}}^{R_{k}} d t q_{i k}(t)(r-t)_{k j}(|r-t|)
$$

for $r \geq S_{i j}$. In these equations $R_{i j}=\left(R_{i}+R_{j}\right) / 2, S_{i j}=\left(R_{i}-R_{j}\right) / 2$, and $R_{m}=\min \left(R_{k i}, R_{k j}-r\right\}$. The $R_{i}$ are range parameters chosen such that $c_{i j}(r)=0$ for $\mathrm{r}>R_{i j}$ and $q_{i j}(r)=0$ for $r \geq R_{i j}$.

Our method for the numerical solution of integral equation approximations in supercritical solutions is as follows:

Step 1. By using the Labik-Gillan method to solve the $\mathrm{OZ}$ equations of mixtures, we can approach the CP to $\rho k T K_{T} \approx 15$. The solutions are $g_{i j}(r), c_{i j}(r)$ and $h_{i j}(r)$. The direct correlation functions are used as input to solve functions $q_{i j}(r)$ in the next step.

Step 2. We use the integral form of equation (11)

$$
\bar{C}_{i j}(r)=\int_{r}^{R_{i j}} d r r c_{i j}(r)=q_{i j}-2 \pi \sum_{k} p_{k} \int_{S_{k j}}^{R_{m}} d t q_{k i}(t) q_{k j}(r+t)
$$

The analytical solution of $q^{\prime}{ }_{i j}(r)$ for hard-spheres (35) is used as an initial guess

$$
\begin{aligned}
& q^{\prime}{ }_{i j}(r)=a_{i} r+b_{i} \quad \text { for } \quad S_{i j}<r<R_{i j} \\
& a_{i}=\left(1-\xi_{3}+3 R_{i} \xi_{2}\right) /\left(1-\xi_{3}\right)^{2}
\end{aligned}
$$




$$
\begin{aligned}
& b_{i}=-3 R_{i}{ }^{2} \xi_{2} / 2\left(1-\xi_{3}\right)^{2} \\
& \xi_{2}=\frac{\pi}{6} \sum_{i} \rho_{i} R_{i}^{2} \\
& \xi_{3}=\frac{\pi}{6} \sum_{i} \rho_{i} R_{i}^{3}
\end{aligned}
$$

and

$$
q_{i j}(r)=\frac{1}{2} a_{i}\left(r^{2}-R_{i}^{2}\right)+b_{i}\left(r-R_{i}\right)
$$

We discretize the functions $q_{i j}(r), q^{\prime}{ }_{i j}(r), c_{i j}(r)$, and $h_{i j}(r)$ into $q_{i j}\left(r_{k}\right), q^{\prime}{ }_{i j}\left(r_{\mu}\right), c_{i j}\left(r_{\mu}\right)$, and $h_{i j}\left(r_{k}\right)$, where $r_{\mu}=u \Delta r$, with $\Delta r=0.04 \sigma_{11}$. To simplify the calculation, we use $R_{1}=2.56 \sigma_{11}$ and $R_{2}=2.56 \sigma_{22}+\left(\sigma_{22}-\sigma_{11}\right)$. Using the trapezoidal rule, we write equation (13) in discrete form

$$
\bar{C}_{i j}\left(r_{k}\right)=q_{i j}\left(r_{\mu}\right)-2 \pi \sum_{k} \rho_{k} \Delta t \sum_{i_{p}=s_{k j}}^{R_{m}} q_{k}\left(t_{p}\right) q_{k j}\left(r_{\mu}+t_{p}\right) \zeta_{p} u=0,1, \ldots\left(R_{i j} / \Delta r\right) .
$$

with $\zeta_{p}=1 / 2$, when $t_{p}$ is at the upper or low limit, else $\zeta_{p}=1$.

Then we applied the NR method to solve equation (18). The Jacobian of equation (18) is

$$
\frac{\partial \bar{C}_{i j}\left(r_{u}\right)}{\partial \bar{q}_{m n}\left(r_{v}\right)}=\delta_{i m} \delta_{j n} \delta_{u v}-2 \pi \Delta t \sum_{k} \rho_{k}\left[\zeta_{v} \delta_{k m} \delta_{i n} q_{k j}\left(r_{u}+r_{v}\right)+\zeta_{v} \delta_{k n} \delta_{j n} q_{k i}\left(r_{v}-r_{u}\right)\right]
$$

The the terms which survive inside the square brackets are as follows:

The first term is nonzero when

$$
R_{k j}-r_{\mu} \geq r_{v} \geq S_{k i}, \text { if } r_{\mu}>\left(R_{k j}-R_{i j}\right)
$$

or

$$
R_{k i} \geq r_{v} \geq S_{k i} \text {, if } r_{k} \leq\left(R_{k j}-R_{k i}\right)
$$

and the second term is nonzero when

$$
R_{k j} \geq r_{v} \geq S_{k i}+r_{u} \text {, if } r_{\mu}>\left(R_{k j}-R_{k i}\right)
$$

or

$$
R_{k i}+r_{u} \geq r_{v} \geq S_{k i}+r_{u} \text {, if } r_{u} \leq\left(R_{k j}-R_{k i}\right)
$$

and $\zeta_{v}=1 / 2$ if $r_{v}$ is at the upper or lower limit, else $\zeta_{v}=1$.

To save time, we used $\Delta r=0.04 \sigma_{11}$ and a short truncated potential to get an approximate solution of $q_{i j}(r)$. As a measure of convergence of the NR cycles, we define the variable $\eta_{1}$ as

$$
\eta_{1} \equiv\left[\frac{\sum_{i=1}^{2} \sum_{j=1}^{2} \sum_{r_{m}=s_{i j}}^{R_{i j}}\left[\Delta q_{i j}\left(r_{u}\right)\right]^{2}}{\sum_{i=1}^{2} \sum_{j=1}^{2} \sum_{r_{m}=s_{i j}}^{R_{i j}}\left[q_{i j}\left(r_{u}\right)\right]^{2}}\right]^{1 / 2}
$$

The NR iteration is stopped when $\eta_{1}$ is less than $10^{-4}$.

Step 3. We interpolate $q_{i j}\left(r_{\mu}\right)$ from step 2 to small grid $\Delta r=0.02 \sigma_{11}$, and extend it to the desired cutoff potential $\gamma_{c u t}=5.12 \sigma_{11}$. Since $q_{i j}\left(r_{u}\right)$ is already a good approximation, direct iteration of equation (20) converges quickly to the final solution. We differentiate functions $q_{i j}(r)$ to get $q_{i j}^{\prime}(r)$. 
It takes a long way to reach this point, but these three steps guarantee accurate solutions of $h_{i j}(r)$, $c_{i j}(r)$ and $q_{i j}^{\prime}(r)$. This set of solutions is then used as a starting point to approach the CP.

Step 4. Using $h_{i j}\left(r_{\mu}\right)$ and $c_{i j}\left(r_{\mu}\right)$ from step 1 and $q^{\prime}{ }_{i j}\left(r_{k}\right)$ from step 3, we have a set of solutions satisfying equations (9) and (10). We proceed with direct iteration of equations (7), (11) and (12). Suppose that $c_{i j}^{\prime}\left(r_{u}\right), h_{i j}^{\prime}\left(r_{k}\right)$ and $q_{i j}^{\prime \prime}\left(r_{u}\right)$ are the $n^{\mu}$ iterates for the functions $c_{i j}(r), h_{i j}(r)$ and $q^{\prime}{ }_{i j}(r)$. The PY closure, equation (5), yields

$$
r_{\mu} h_{i j}^{\text {OuT }}\left(r_{u}\right)=f_{i j}\left(r_{u}\right)\left[r_{u} h_{i j}^{n}(r)+r_{\mu}-r_{u} c_{i j}^{n}\left(r_{k}\right)\right]-r_{\mu}
$$

where

$$
f_{i j}\left(r_{k}\right)=\exp \left[-u_{i j}\left(r_{k}\right) / k T\right] \text {. }
$$

The $(n+1)^{n t}$ approximations for $h_{i j}^{N E W}\left(r_{\mu}\right)$ is given by damping functions of $h_{i j}^{n}\left(r_{\mu}\right)$ and $h_{i j}^{O U T}\left(r_{\mu}\right)$.

$$
h_{i j}^{N E W}\left(r_{\mu}\right)=\alpha h_{i j}^{O U T}(r)+(1-\alpha) h_{i j}^{n}\left(r_{\mu}\right)
$$

where $\alpha$ is a mixing parameter designed to ensure convergence. The damping is only required for following region.

$$
\sigma_{i j} \leq r \leq 1.6 \sigma_{i j}
$$

Using equation (10) to solve $h_{i j}^{n+1}\left(r_{k}\right)$.

$$
r_{\mu} h_{i j}^{n+1}\left(\left|r_{\mu}\right|\right)=-q_{i j}^{i n}\left(r_{\mu}\right)+2 \pi \sum_{k} \rho_{k} \int_{S_{k}}^{R_{u}} d t q_{i k}^{n}(t)\left(r_{\mu}-t\right) h_{k j}^{N E w}\left(\left|r_{\mu}-t\right|\right)
$$

The functions $q_{i j}^{i n+1}\left(r_{\mu}\right)$ are updated by adding the difference of $r_{\psi} h_{i j}^{n+1}\left(r_{\mu}\right)$ and $r_{\mu} h_{i j}^{N E W}\left(r_{\mu}\right)$ with the damping factor $\beta$.

$$
q_{i j}^{i n+1}\left(r_{\mu}\right)=\beta\left[r h_{i j}^{n+1}\left(r_{\mu}\right)-r h_{i j}^{N E W}\left(r_{k}\right)\right]+q_{i j}^{i n}\left(r_{\mu}\right)
$$

Then we can integrate $q_{i j}^{\prime n+1}(r)$ to get $q_{i j}^{n+1}(r)$.

The $c_{i j}^{n+1}\left(r_{k}\right)$ are solved by equation (9)

$$
r_{k} c_{i j}^{n+1}\left(r_{\mu}\right)=-q_{i j}^{i n+1}\left(r_{k}\right)+2 \pi \sum_{k} \rho_{k} \int_{S_{k}}^{R_{m}} d t q_{k}^{n+1}(t) q_{k j}^{i n+1}\left(r_{k}+t\right)
$$

We checked the quantity $\eta_{2}$, defined by

$$
\eta_{2} \equiv\left[\frac{\sum_{i=1}^{2} \sum_{j=1}^{2} \sum_{k=1}^{N}\left[\Delta q^{\prime}{ }_{i j}\left(r_{k}\right)\right]^{2}}{\sum_{i=1}^{2} \sum_{j=1}^{2} \sum_{k=1}^{N}\left[q^{\prime}{ }_{i j}\left(r_{k}\right)\right]^{2}}\right]^{1 / 2}
$$

The DI cycle was stopped when $\eta_{2}$ was less than $10^{-6}$. If equation (34) is not satisfied, then we return to equation (4.63).

In 1992, Busigin and Phillips[8] proposed an implicit Euler's method for the Newton-Raphson algorithm. Their method is particularly suited to "stiff" equations, thus affording high stability.

\section{Solute-Solvent Correlation in Attractive and Repulsive Mixtures}

The solvent-solute (solvation) structure is central to supercritical behavior. In this section, we examine the integral equation results for attractive and repulsive SCF. We use Lennard-Jones potential to model the solvent and solute interactions. This representation gives correct qualitative behavior (but not quantitative information). But this is what we are interested in. 


\subsection{ATTRACTIVE MIXTURES}

Petsche and Debenedetti (27) carried out computer simulation for both aturactive and repulsive solutions, with parameters simulating the Ne.Xe system.

Parameters for neon and xenon used in simulation *

\begin{tabular}{lcc}
\hline$i=$ & Neon & Xenon \\
\hline Size ratio $\sigma_{i} / \sigma_{N_{e}}$ & 1 & 1.435 \\
Energy ratio $\varepsilon_{i} / \sigma_{N_{e}}$ & 1 & 7.04 \\
\hline
\end{tabular}

* The MD usod 864 particles with 863 solvent molecules and 1 solute molecule.

We have solved the HMSA closure for the attractive Ne.Xe case at the followinb conditions:

$$
\begin{array}{ll}
T^{*}=1.4 & \rho^{*}=0.35 \\
T^{*}=1.4 & \rho^{*}=0.8
\end{array}
$$

Figures 5.1 and 5.2 show the solvent-solute correlation functions $8{ }_{12}(r)$. One observes that at $T^{*}=1.4$ and $\rho^{*}=0.35$ (near the solvellt CP), $8_{12}$ decays very slowly from above unity. (exhibiting a persistent tail $>1$ for $\left.r>4 \sigma_{11}\right)$. This slow decay is called a long-range buildup. This buildup disappears at a higher density $\left(\rho^{*}=0.8\right)$. This behavior has been alluded to earlier [9]. We have also made calcula. tions using the RHNC closure [10] for the carbon dioxide-pyrene mixture studied by Brennecke et al. [11] The solute pyrene is very dilute in $\mathrm{CO}_{2}$ (mole fraction $3 \times 10^{-7}$ ). The results for the three correlation functions: solvent-solvent, solvent-solute, and solute-solute are shown in Fig. 5.3. Again we clearly see the long-range buildup of the solvent-solute correlations. This buildup translates into a large value for the fluctuation integral $G_{12}$. We note that $G_{12}$ is closely related to the excess coordination number $N_{i}^{e x}$ (now depending on the range of integration $L$. See also eq.(3.21)).

$$
N_{i}^{\beta x}(L)=\rho_{1}^{0} \int_{0}^{L} d r 4 \pi r^{2}\left[g_{12}(r)-1\right]
$$

This number gives the excess of solvent molecules 1 radially surrounding the central solute molecule 2 up to a radial distance of $L$. For randoin distributions, $N i_{2}(L=\infty)=0$, meaning that there is no excess of solvent molecules around the center. The density of solvent molecules is given simply by the uniform density $\rho_{1}$ and its radial number is given by $(4 \pi / 3) \rho_{1} L^{3}, L$ being the coordination distance from the center. When Nex 1 or $G_{12}$ is greater than zero, there is positive correlation of 1 around $2 ;$ less than zero, negative correlation of 1 around 2. Figure 5.4 shows the $G_{i j}$ for three types of fluids: (A) attrac. tive mixture $G_{12},(\mathrm{P})$ pure solvent $G_{11}$, and $(\mathrm{R})$ repulsive mixture $G_{12}$ at $\mathrm{T}^{*}=1.35$ and $\rho^{*}=0.4$ (all variables reduced according to solvent parameters). The curves represent the excess numbers of solvent neighbors at increasing coordination distance $L$ for the solvent-solute correlation. Clearly, for case $A$, $G_{12}$ becomes very large $(-110)$, indicating substantial clustering of solvents around the solute molecule. This value sould be compared with the pure solvent-solvent $G_{11}$ of -8 , and "repulsive" solvent-solute $G_{12}--6$. We note that the major contribution to excess number comes from long range buildups. Namely, large clusters did nol derive from first neighbors alone. Upon reflection on geometry, this stands to reason since there is only so much room in the first neighbor shell for packing molecules. For clusters of size approaching 100 , they must come from second, third, and higher neighborhoods.

\subsection{REPULSIVE MIXTURES}

Similar calculations are carried out for the "repulsive" mixtures. This time, infinitcly dilute neon is dissolved in supercritical xenon. The following conditions are used: 


$\begin{array}{ll}T^{*}=1.4 & \rho^{*}=1.35 \\ T^{*}=2.0 & \rho^{*}=0.35 \\ T^{*}=1.4 & \rho^{*}=0.80 \\ T^{*}=1.34 & \rho^{*}=0.27\end{array}$

The results are plotted in Figs. 5.5(a) and (b). At high density $p^{*}=0.8$, the pef $g_{12}$ is oscillatory. Near the CP of xenon, $8_{12}(r)$ becomes suppressed in magnitude after the first peak. The second peak barely makes above unity $(\sim 1.007)$. At a lower temperature $\left(T^{*}=1.34\right)$, the second peak is less than 1 (see Fig. 5.5(b)). Thenceforth, the correlations do not rise above 1, representing undercorrelation between the neon solute and the xenon solvent. This behavior is exactly the opposite of the aturactive case discussed above. The solvent molecules instead of densifying around the solute molecule, stay away (cav. itate) from neon. This behavior is cicarly seen in $G_{12}$. Figure 5.6 gives the $G_{12}$ for four "repulsive" mixtures. The deficit in solvent molecules as the critical point of xenon (in PY approximation, $T_{c}{ }^{*}=1.29, \rho_{c}^{*}=0.27$ ) is approached reaches --12 for the lowest temperature studied. Again, the deficit is made out of both the shor-range local density depletion and long-range debih, with the major part coming from long-range solvent-solute undercorrelation. We have compared our PY calculation with the simulation data of Petsche et al. (27)(Fig. 5.7). While there is statistical scatter in the simula. tion data, the two results reinforce each nther in the height of the first peak and locations of the peaks and valleys.

By using integral equations, solved for model supercritical mixtures, we are able to show on the molecular level the densification and cavitation of solvent molecules around the solute molecules depending on whether the mixture is "uttractive" or "repulsive". The story does not end here. Further interesting structural manifestations are found for the solute-solute correlations and are discussed next.

\section{Solute-Solute Aggregation}

Not only do solvent molecules tend to cluster about solute molecules in attractive mixtures, we also find a high degree of solute-solute aggiegation near the CP of the solvent. We shall discuss the aturactive case first, then the repulsive case next. Both cases show interesting solute-solute correlations.

\subsection{ATIRACTIVE MIXTURES}

Figure 6.1 shows the correlation function $82(r)$ for the $\mathrm{LJ}$ mixture simulating the carbon dioxide-naphthalene system which is inaccessible by computer simulation and has bcen oblained only by integral equation methods. For the attractive mixture, the solute-solute pair distributions exhibit increased height of the first peak near the $C P$, indicating increased short-range solute concentration about a solute molecule, consistent with interpretations of the excimer fluorescence spectra by Brennecke and Eckert $(25,26$ ) (see also Bright). This short-range solute-solute pair structure is similar to but larger in magnitude than the short-range solute-solvent structure presented eartier. Likewise, the longrange solute-solute pair structure increases near the CP suggesting a solute-solute cluster commingled with the solvent-solute cluster. Because the solute concentration is very small, the solute-solute cluster is only a statistical association. Despite the low mole fraction, this statistical solute-solute cluster could be expected to exhibit macroscopic effects, for example, in solute-solute dimerization reactions, near the $C P$. In cases when the mrrelation length becomes large (i.e., near the $C P$ ) the notion of dilute solutions being approximated as infinitcly dilute must be employed with great caution since despite the rarefaction of solutes, detectable aggregates do occur.

In addition to showing increased height of the first maximum near the $\mathrm{CP}$, the shor-range solutesolute pair distributions for attractive inixtures (Fig. 6.1) show a much shallower minimum between the first and second maxima; this is similar to the behavior of the solute-solvent pair distributions near the $\mathrm{CP}$ but much exaggerated. As mentioned earlier, the fluorescence spectroscopic experiments of Bren. necke and Eckert $(25,26)$ indicated significant excimer formation for very dilute pyrene dissolved in supercritical cthylene and carbon dioxide. We shall report now some additional integral cquation calculations for the carbon dioxide-pyrene system. The following Lennard-Jones parameters are used: 


\begin{tabular}{lcc}
\hline Parameler & G/k K & O A \\
\hline $\mathrm{CO}_{2}-\mathrm{CO}_{2}$ & 225.3 & 3.794 \\
Pyrene-pyrene & 662.8 & 7.14 \\
$\mathrm{CO}_{2}$-pyrene & 386.4 & 5.467 \\
\hline
\end{tabular}

The pressure used is 82.5 bar (or $1197.1 \mathrm{psia}$ ), concentration $y_{\mathrm{pr}}=3.0 \times 10^{-7}$, and density (pure $\mathrm{CO}_{2}$ ) $=$ $0.8059 \mathrm{lbmole} / \mathrm{cuft}$. The RHNC equations (39) were solved for this case. The three pair correlation functions $g_{11}, 8_{12}$, and $g_{22}$ are shown in Fig. 5.3.

The solule-solute correlation function shows a curious double peak with a shallow minimum between. We shall infer that this fcature (first minimum greater than unity) characterizes the affinity of pyrene molecules for one another. To determine the origin of this shallow minimum, we need to exam. ine the geometry of packed molecules. For this case the radial packing geometry is shown in Fig. 6.2. The first peak of $g 2(r)$ represents the direct contact between a pair of pyrene molecules (direct pyrene-pyrene correlation). The second peak represents the geometry of a pair of pyrene molecules sandwiching a $\mathrm{CO}_{2}$ molecule in between (pyrene-pyrene correlation mediated via a solvent molecule: i.e., pyrene- $\mathrm{CO}_{2}$-pyrene). There is a perceptible shoulder after the second peak. It is the normal location for triplet pyrene correlations (concatenations such as: pyrene-pyrene-pyrene). Far from the $\mathrm{CP}$, the first minimum in correlation functions usually falls below 1.0 because of the volume excluded by an intervening solvent molecule. This does not happen here. In this case at $r=2.7 \sigma_{11}$, where normally a $\mathrm{CO}_{2}$ molecule would intervene between the pyrene molecules, the usual gap with $822<1.0$ fails to occur. Cerainly, this is not due to scarcity of solvent molecules (as pyrene is infinitely dilute in $\mathrm{CO}_{2}$ ). This could be explained by the aggregation of pyrene molecules at this state near the $C P$, in such a way that causes exclusion of $\mathrm{CO}_{2}$ and simultancously relative enrichment of pyrene molecules. This behavior is consistent with and strengthens the picture of excimer formation.

To determine how dilute is dilute, we have also calculated a case at a smaller concentration of pyrene (same temperature and pressure) $y_{p r r}=2.35 \times 10^{-11}$. Very similar $g_{i j}(r)$ are obtained. In fact, the differences in the numerical values of $g_{i j}(r)$ from the two concentrations are in the fourth decimal place.

We also examined the tempcrature effect on radial structure. Figures 5.3 and 6.3 (a) (b) show the three radial distribution functions 811,812 and 822 at three temperatures $-T^{\circ}=1.37,1.50$, and 2.00 . Besides the ordering of peak heights, the other noticcable feature is the deepening first minimum of 822 as the temperature is increased. Starting at $8_{22} \approx 2.0$ (enrichment), the value at the minimum firally drops $108_{22}=1.0$ at the highest temperature $\left(T^{*}=2\right.$ ). Thus, the aggregation (detected by lack of exclusion of $\mathrm{CO}_{2}$ molecules) of pyrene molecules is reduced at higher temperatures when conditions are farther removed from the CP.

We find that these solute-solute correlations show intriguing isomorphism to the correlation functions observed for diatomics (while we want to make the distinction here that excimers are definitely not covalent dimers). To contrast the structural idiosyncrasies with the diatomics, we carried out RISM calculations for dimer-monomer mixtures. For the monomers $\left(\mathrm{CO}_{2}\right)$ and dimers, the following $\mathrm{LJ}$ parameters are used

\begin{tabular}{lccc}
\hline Parameter & & $\varepsilon / k \mathrm{~K}$ & $\sigma \mathrm{A}$ \\
\hline monomer-monomer & $\mathrm{mm}$ & 225.3 & 3.794 \\
dimer site-dimer site & $\mathrm{ss}$ & 225.3 & 5.691 \\
monomer-dimer site & $\mathrm{ms}$ & 225.3 & 4.743 \\
\hline
\end{tabular}

The bond length between the sites of the dimer is taken to be $l=0.66 \sigma_{m m}=2.5 \AA$.

Figure 6.4 shows the site-site correlation functions, $8 \mathrm{~mm}, 8_{\mathrm{ms}}$, and $8 \mathrm{ss}$ (site refers to an atom in the dimer). The first peak occurs at the collision diameter of the sites in two dimers $\left(\approx 1.68 \sigma_{\mathrm{mm}}\right)$. The second peak is due to one site of the first dimer with the other (farther) site of a second dimer, $r=2.34$ $\sigma_{m m}$. We shall designate one sitc of the first dimer as the "host", the close-by site in the second dimer 
as the "guest", and the farther site in the second dimer as the "companion" of the guest. In this language, the the second peak is duc to the correlation between the host and the other companion. The increment in distance is, of course, the bond length $0.660 \mathrm{~mm}$. Actually, this peak oxcurs at a shorter distance because of all possible nonlinear angular approaches which have a tendency to reduce the dis. tance from 2.34 to about $2.20 \mathrm{~mm}$. The third peak comes from a pair of siles mediated by a monomer molecule, and occurs al $=2.80_{\mathrm{mm}}$.

The interesting part is the similarity in the qualitative features of $8 u$ with those of the low tem. perature pyrene-pyrene 82 . The tirst minimum is shallow and much greater than unity. This phenomenon has been explained geometrically earlier. If we focus our attention on the correlation between the site called the host in the first dimer and the companion in the second dimer, then it is clear that in between these two, there is the neighbor site of the second dimer that intervenes and excludes a solvent molecule from entering. This explains the probabilistic "enrichment" that forms the shallow first minimum; i.e., probabilistically, it is highly likely to have a neighbor - from a sile in the second dimer - localized here, with a probability density above and beyond the average density of dimers. The same was observed for the low temperature $\mathrm{LJ}$ mixtures modeling $\mathrm{CO}_{2}$-pyrene. If pyrene and pyrene tend to aggregate, then there is a increased likelihood of having a third pyrene molecule sandwiched between a host pyrene molecule and a companion pyrene molecule. This third pyrene molecule is tied 10 the companion pyrene molecule because of aggregation. There are of course differences between the structures of the monomer-dimer case and the $\mathrm{CO}_{2}$-pyrene case. The three peaks in the former are due to host-guest, host-companion, and host-solvent-guest correlations; whereas the peaks for $\mathrm{CO}_{2}$-pyrene are due to correlations of host-gu'st, host-solvent-guesh and host-guestsecond guest.

\subsection{REPUISIVE MIXTURES}

It is of interest to investigate the solute-solute interactions in the repulsive mixtures discussed above. We have seen the cavitation of the solvent molecules away from the solute molecule in the Ne.Xe sysiem. How do the solute molecules (in this case neon) behave toward each other? We have calculated the 822 for the four state conditions listed in Section 5.2. The behavior is shown in Figs. 6.5 (a) and (b). As the density of solvent (xenon) is lowered from 0.8 to 0.35 , the oscillations in Ne-Ne correlations are damped. If we calculate the excess number (eq.(6.1), see Fig. 6.6), we note that there is enrichment of excess numbers when the critical condition of xenon is approached. At high temperatures, T* 2.0, there is deficit in neon-neon correlations $\left(G_{2}<0\right)$. At high densities, $G_{2}$ rises above zero. Namely, neon molecules tend to "separate" out of the mixture. Near the critical point of xenon, the "separation" increases rapidly. Since neon molecules are "reja led" by the xenon solvent, evidenced by the solvent cavitation discussed earlier. Ne tends to form aggregates too. By analogy with nonpolar molecules dispersed in aqueous medium, this aggregation is caused by xenon-phobic attraction (cf. sic hydrophobic aturaction). If we compare the solute-solute aggregation in repulsive mixtures with that in the attractive case, our calculations show that the former is several orders of magnitude smaller than the latier. The excess $G_{22}$ for the attractive case is on the order of $10^{3}$ near $\mathrm{CP}$.

The algorithm we have used for solving the $\mathrm{OZ}$ equations of mixtures near the $\mathrm{CP}$ has proved to be robust and efficient compared with other methods we have tried. The results have been shown to agree closely with results from the efficient Labik-Gillan method, but the new method can approach the $\mathrm{CP}$ much more closely. The $\mathrm{CP}$ for dilute $\mathrm{IJ}$ mixures can be located quite precisely. The algorithm is applicable quite generally to mixtures the components of which may be modeled as interacting with a pair potential which is finite in range.

Using integral equation theories, we are able to demonstrate here the detailed microscopic behavior of the interesting repulsive supercritical mixtures proposed erstwhile by Debenedetti (28). The results of the correlation functions have proved helpful in gaining understanding of the distribution of molecules surrounding a central solute molecule in dilute supercritical solutions. These results are consistent with observed macroscopic properties which are sensitive to the shon-range structure -- particularly spectroscopic results. The long-range solute-solvent structure for attractive mixtures near the $C P$ from integral equation calculations is consistent with the thermodynamic properties which depend on the solute-solvent fluctuation integral - solute partial molar volume and solubility. Long-range 
structure is inaccessible by compucer simulation techniques.

The solute-solute distributions for attractive mixtures from integral equation calculations are also consistent with the observation of a high proportion of solute excimers near the CP in very dilute super. critical solutions. To date there are no solute-solute distributions near CP trom molecular simulation calculations (which have been performed at infinite dilution). Nor are there results with which to com. pare the long-range solute-solute pair distributions. Judging by the integral equation results, one mig'it expect dramatic effocts on other applications such as chemical reactions in supercritical media.

\section{Thermodynamic Behavior of Attractive/Repulsive SCF}

In previous sections, we have exhibited the structural changes 812 and 822 accompanying the attractive and repulsive mixtures. In this section, we shall look into the consequences as reflected in the partial molar volumes and excess numbers for both mixtures. (The third class, the weakly attractive mixtures of Debenedeal [7], will not be examined here.)

\section{ATTRACTLVE MIXTURES}

We first look at the partial molar volumes. We observe that neas the $C P, K_{T}$ is large and positive. However, for attractive mixtures, $N \mu^{x}$ also increases rapidly. When $N \mu^{a}$ overtakes $\rho_{i}^{0} k T K_{T}, V_{2}^{*}$ becomes negative according to eq.(3.23). The values could reach $-8000 \mathrm{cc} / \mathrm{gmol}$ (for naphthalene in carbon dioxide). This bchavior has bcen observed experimentally (2). Concomitant with the negative PMV, there is an increase of the solubility of naphthalene in $\mathrm{CO}_{2}$. This could be deduced from $\propto$ Q. (3.19) or from the enhancement factor, $E$. Cochran, Prund, and Lce [12] showed that

$$
\ln E=\ln \left(y_{2} P / p^{\text {sat }}\right)=\frac{1}{k T} v q_{2}\left(P-p_{v}\right)+\frac{1}{k T} \int_{P_{v}}^{p} d P G_{12}+\ln z
$$

Thus when the solute-solvent factor, $G_{12}$ increases, the solubility will also increase.

We have used the Lennard.Jones $(\mathrm{L})$ polentials to model the solute naphthalene and the solvent $\mathrm{CO}_{2}$ [13]. The pcr's $\mathrm{s}_{11}$ and $\mathrm{8}_{12}$ were decermined by the PY equation. For this patently attractive mix. ture, the results are shown in Fig.7.1 for different densities $\left(p^{*}=p_{11}^{3}\right.$ from 0.27 to 0.50 , and $T^{*}$ $=k T / \varepsilon_{11}=1.415$ ). Note that for $P Y$, the critical temperature of $L$ fluids in molecular units is -1.29 , and critical density is -0.27 . (This can be compared with the simulation critical dats of $L J$ potential 1.31 and 0.31 , respectively). Clearly, there is increase of the first peak as the density is lowered from 0.42 downward loward the critical value 0.27 . Secondly, beyond the first peak, $8_{12}$ becomes enhanced and exhibits a persistent long-range tail (with values above unity). These are the two characteristics for attractive supercritical mixtures mentioned earlier.

In sum, the evidence shows that (i) there is local enhancement of solvent molecules, as judged from the higher first peak of $8_{12}$; however, (ii) the major buildup in $G_{12}$, and thus $N_{1}^{2}$, comes from 20 neighborhoods'.. long range buildups (see Fig.5.4). This buildup contrasted with the pure neon fluid (Curve $P$ ) is quite dramatic. The excess number of pure neon only reaches -7.4 overall ${ }^{2}$ (cf. 110 for curve A). (We shall discuss the repulsive case, $R$, later.)

For a similar aturactive mixture, dilute naphthalene (mole fraction $=10^{-9}$ ) dissolved in $\mathrm{CO}_{2}$, we have calculated $\vec{V}_{2}$ (eq. (3.8)) with the Lennard-Jones potential model. Results are compared with the data of Ecken [2] in Fig. 7.2. For the potential paranelers given, the low density branch is well predicted. The high density branch shows some discrepancies. Similar observations are given by McGuigan and Monson [6]. Clearly, the $\mathrm{LJ}$ potential is not a good model for $\mathrm{CO}_{2}$ and naphthalene.

\footnotetext{
'About 20 molecular dinmerers. "gain".

The excess number in pure neon is due to purely colvent-solvent correlations. At the given state condition, there is a net
} 
PY also introduces some errors. However, model potentials give qualitatively universal behavior for real mixtures and are worth further study. To determine the PMV behavior at different temperatures, we plot five isotherms in Fig. $7.3(b) .\left(T^{*}=1.642,1.465,1.382,1.342\right.$, and 1.334). We see a continuous growth of the negativity of the PMV, as the critical temperature $(T=1.29)$ is approached. We also plot $N_{i}^{2 z}$ in Fig. 4(a). There is a corresponding growth in $N_{i}^{\alpha z}$. Eq.(3.23) indicates that for nega. tive PMV to happen, the difference between $p_{1} k T K_{T}$ and $N_{i}^{2}$ must be negative (or $C_{\overline{12}}>1$ ). The excess number of solvent molecules surpasses the compressibility. Adding a solute molecule to the solution will attract more solvent molecules (condensation) than increasing the bulk volume (expansion).

\section{REPULSIVE MLXTURES}

For repulsive mixtures, the excess number of solvent molecules about the solute becomes negative (a deficit). The conditions for this behavior were given in Petsche and Debenedetti [14]. For dilute neon dissolved in a xenon solvent, we have the repulsive behavior. The strength of interaction for neon-xenon $\left(\varepsilon_{12}<\varepsilon_{11}\right)$ is weaker than the xenon-xenon (1.1) interaction (2.65 times less). When the CP is approached, the influence of this energy disparity on solvent structures is magnified. Xenon molecules are pulled away from the neon solutes due to cohesion, and leaving a correlation hole (we call it here cavitation). Since there is depletion of solvent molecules around the solute, the excess number becomes negative. The curve $R$ in Fig.5.4 shows that $N_{1}^{a x}--6$.

From eq. (3.8), we see that the PMV will be positive. Fig. 7.4(b) gives the $v_{2}^{*}$ for dilute neon $(=2)$ in a xenon solvent $(=1)$ at three temperatures $\left(T^{*}=2.0,1.4,1.34\right)$. They are all positive. There is a direct correspondence between the $N_{1}^{2}$ and $\nabla_{2}^{*}$ values. The case is in vivid contrast to the attractive mixtures of Figs. 7.3 where the $N_{i}^{2 \pi}$ is positive and PMV negative. (For the weakly attractive mixtures, $N_{\mu}^{\mu}$ is positive but small, thus the PMV remains positive.) We note that in Fig.7.4, the size of $p_{1} \nabla_{2}^{*}(+$ 15) for repulsive mixtures is an order of magnitude smaller than the attractive case Fig.7.3 $\left(p_{1} v_{2}^{-\infty}=-150\right)$.

We have demonstrated, using integral equation theories, that the cavilation and/or condensation of solvent molecules around the solute molecules are responsible for the repulsive or attractive behavior of supercritical mixtures. This work provides added proof to earlier simulation and theoretical studies. We have examined the behavior through the molecular distribution functions, especially with respect to the long-range contributions to the solvent-solute $K B$ factor $G \tilde{12}$. The macroscopic manifestation of negative partial molar volume near the critical point is due, for the most part, to long-range persistent solvent-solute correlations. Observations on the repulsive mixtures show that cavitation of solvent molecules over long distances is responsible for their unusual behavior. The distinction between attractiveness and repulsiveness can be gauged in the case of Lennard-Jones potentials by employing similar analyses given by Pelsche and Debenedetti (PD) (13) for van der Waals gas. Near the critical point of the solvent $\left(T_{r}=1, p_{r} \approx 1\right)$, the mixture exhibits attractive behavior if

$$
\phi=\frac{\alpha^{1 / 2}}{1+(\gamma-1) / 3}>1
$$

The parameters $\alpha$ and $\gamma$ can be estimated in the $\mathrm{L}$ case by $\alpha=\left(\varepsilon_{22} \sigma_{22}^{3} / \varepsilon_{11} \sigma_{11}^{3}\right)$, and $\gamma=\left(\sigma_{22}^{3} / \sigma_{11}^{3}\right)$. For the parameters used for the $\mathrm{CO}_{2}$-naphthalene mixture, $\phi=1.545$, greater than 1 . The behavior is attractive. For dilute xenon (the solute) dissolved in neon (the solvent), $\phi=2.76>1$. Again, we have attractive behavior. However, for repulsive mixtures,

$$
\psi=\frac{3 \alpha^{1 / 2}}{(1+\gamma)-\frac{1}{3}}<1
$$

Applying this 10 dilute neon (the solute) dissolved in xenon (the solvent), $\psi=0.654<1$. We have repulsive behavior, as predicted by the PD rules. We note that the $\alpha$ value of xenon over neon is very large, about 20.8, mostly due to the energy ratio (7.04). Thus the Ne.Xe mixture behavior is accounted for essentially by energy disparity. Stronger solvent-solute affinity promotes aggregation of solvent molecules around the solute molecules (solvation). On the other hand, stronger solvent-solvent affinity promotes self-association (cohesion) among the solvent molecules and exclusion of the solute molecules. 


\section{Synergistic Effects of Entrainers: Ternary Systems}

When methanol, as a cosolvenh is added to the $\mathrm{CO}_{2}-2$-naphthol mixture, the solubility of 2 . naphthol is increased fivefold (see Fig. 8.1) over the solubility in the binary $\mathrm{CO}_{2}$-2-naphthol system. This greatly enhanced solubility of 2-naphthol is what we refer to as synergism of the cosolvent methanol for the solute. Not all cosolvents entall synergism, there are ones causing "interferences": namely, addition of cosolvents or cosolutes depresses the solubility. In this section we shall present the formulas for treating temary SFC.

We define a solution, solutes 2 in the solvent 1 , as infinitely dilute if $x_{2}$ is strictly zero; as dilute, if the first order dependence on $x_{2}$ is valid (i.e., Henry's law region). For ternary systems with solvent 1 , solute 2, and cosolvent (or cosolute) 3, same definition applies to species 3. We generalize the results for binaries as given by Debenedetti-Kumar and Cochran-Lee to temaries. In the dilute range, the fugacity coefficient for species 2 has been given by Jonah-Cochran as

$$
\ln \phi_{2}=\ln \phi_{2}{ }^{\infty}+x_{2} K_{22}+x_{3} K_{23}
$$

where the parameters $K_{i j}$ are defined as the Taylor expansion coefficients:

$$
K_{23}=\frac{\partial \ln \phi_{2}}{\partial x_{3}}
$$

which has been shown to be equal to the second derivative

$$
K_{23}=\left.\frac{\partial \ln \phi_{2}}{\partial x_{3}}\right|_{\tau, \rho, x^{\prime}}=-\left.\frac{\partial^{2} \ln \phi_{1}}{\partial x_{2} \partial x_{3}}\right|_{T, x^{\prime \prime}}
$$

Cochran and Lee have shown that the $K_{i j}$ can be expressed in terms of the KB factors; e.g.

$$
\begin{aligned}
& K_{22}=-\rho_{1}^{0}\left[G_{11}^{0}+G_{22}-2 G_{12}\right] \\
& K_{23}=-\rho_{1}^{0}\left[G_{11}^{0}+G_{20}-G_{12}^{\circ}-G_{13}\right]
\end{aligned}
$$

Chialvo [15] has expressed these quantities in terms of the DCFI:

$$
K_{22}=1-C_{22}^{\ddot{2}}-\rho_{1}^{0} k T K_{T}\left(1-C_{12}\right)^{2}
$$

Similarly,

$$
K_{23}=1-C_{23}-p_{1}^{0} k T K_{T}\left(1-C_{12}\right)\left(1-C_{13}\right)
$$

Thus the solubility of 2 in the SCF in the presence of a pure condensed phase (solid phase of 2) is

$$
P \phi_{2} x_{2} \operatorname{xxp}\left[K_{22} x_{2}+K_{23} x_{3}\right]=f \xi
$$

And for 3

$$
\left.P \phi_{3} x_{3} \exp \left[K_{33} x_{3}+K_{22} x_{3}\right]=f\right\}
$$

Thus if $K_{23}<0$, addition of solute 3 will increase the solubility of 2 (compared to the binary $1+2$ ). There is "synergism" between the solute and the cosolute. On the other hand, if $K_{23}>0$, then additions of 3 depresses the solubility of 2 . This is due to the "interference" of the cosolute. The results of Fig. 
8.1 show that methanol, the cosolvent clearly belongs $w$ the "synergistic" category. Kumik and Reid have shown that the cosolute 2,3-dimethylnaphthalene in the CO2-phenanthrene mixture gives "negative" syncrgy, reducing the solubillity of phenanthrene: an "interference" effect

We note that these analyses are valid only in the "dilute" (Henry's law) region. For more concentrated solutions, higher order Taylor's terms are needed. If we consider the $x_{2} \rightarrow 0$ limit (i.e., infinite dillu. tion) as the primary contribution to SCF, where most studies in SCF mixtures belong, then the analyses represented by the $K_{i j}$ terms are secondary contributions, disappearing when $x_{2} \rightarrow 0$.

\section{Adsorption from SCF: Fluld Structure near Solid Surfaces}

Adsorption of supercritical fluids is used in a number of separation processes such as adsorption from a SCF solution or desorption into a SCF solution in regeneration of sorbents with SCF, SCF chromatography, and SCF extraction of hazardous chemicals from contaminated soils. The work described in this section was motivater by the importance of adsorption and desorption in these separa. tion processes. The fundamental understanding of adsorption of SCF can be achieved by examining the molecular distributions near a wall.

Fundamental, molecular study of adsorption has employed the techniques of density functional theory (c.g. Ebner et al.), integral equation theory or molocular simulation. In the present work, techniques based on integral equation theory will be employed. In the simplest techniques for application of iniegral equation theory, due to Henderson, Abraham, and Barker (HAB), the wall(W)-particle(A) Ornstein-Zernike equation

$$
h_{A W^{\prime}}(z)=c_{A W}(z)+2 \pi p_{A} \int_{-}^{\infty} d i c_{A W}(1) \int_{1:-11}^{\infty} d s s h_{M}(s)
$$

is solved (with an appropriate closure approximation) under the approximation that $h_{M}(s)$ in $(9.1)$ is taken as equal to the homogeneous total correlation function for the bulk fluid. The HAB integral equation approach is known (Finn and Monson) 10 produce results with important qualitative and quantita. tive failures. In contrast, the Henderson-Plischke-Sokolowski (HPS) approach (9.4) solves the inhomogeneous Ornstein-Zemike equation for the wall-particle system in Fourier space

$$
\bar{h}\left(z_{1}, 2, k\right)=\bar{c}\left(z_{1}, z_{2}, k\right)+\int d z^{\prime} \rho\left(z^{\prime}\right) \bar{c}\left(z_{1}, z_{1}, k\right) \bar{h}\left(z_{1,2}^{\prime} k\right)
$$

where

$$
\begin{aligned}
\bar{f}\left(z_{1}, z_{2} k\right) & =\int d^{2} r f\left(z_{1}, z_{2}, r\right) \exp [i k \cdot r] \\
& =2 \pi \int d r r f\left(z_{1}, z_{2}, r\right) J_{0}(k r)
\end{aligned}
$$

with an appropriate closure approximation plus one of several exact expressions for the inhomogeneous density in terms of the correlation functions. Solution of the inhomogeneous HPS equation is a much more demanding computation in terms of required memory and CPU cycles, but is much more accurate than solution of the HAB equation.

For solute $B$ at infinite dilution in the solvent $A$, the HPS equation takes the form $(A+B / W)$ :

$$
\bar{h}_{M}\left(z_{1}, z_{2}, k\right)=\bar{c}_{M}\left(z_{1}, z_{2}, k\right)+\int d z^{\prime} p_{A}\left(z^{\prime}\right) \bar{h}_{M}\left(z_{1}, 2, k\right) \tau_{A M}\left(z_{2}^{\prime}, 2, k\right)
$$

and

$$
\bar{h}_{A B}\left(z_{1}, z_{2} k\right)=\bar{C}_{A B}\left(z_{1}, z_{2} k\right)+\int d z^{\prime} \rho_{A}(z) \bar{h}_{A A}\left(z_{1}, 2, k\right) \bar{C}_{A B}\left(z_{1}^{\prime}, z_{2} k\right)
$$

In the present work we have applied the HPS approach to study adsorption equilibrium in systems where the fluid phase is either a pure SCF or a dilute solution of a solute in a fluid near the critical 
point of the pure solvent. Earlier work in this area (Plischke and Henderson) was limited in the number of grids used in the numerical algorithm by the fast memory on the available compuler. In the present work, we have extended the number of grids and tested the sensitivity of our results to this numerical parameter. After thus testing the numerical algorithm, we have studied several cases of adsorption equilibrium between surface and a SCF.

\section{CALCULATIONMETHOD}

In numerical calculations it is often desirable to replace the variable $h$ by the variable $\gamma=h-c ;$ in this form equations (9.4) and (9.5) become

$$
\begin{aligned}
\bar{\gamma}_{M}\left(z_{1}, z_{2}, k\right)= & \int d z^{\prime} \rho_{A}\left(z^{\prime} \bar{\gamma}_{M}\left(z_{1,2}, k\right) \bar{\tau}_{M}\left(z^{\prime}, z_{2}, k\right)\right. \\
& +\int d z^{\prime} \rho_{A}\left(z^{\prime}\right) \tau_{M}\left(z_{1}, 2, k\right) \tau_{M}\left(z^{\prime}, z_{2}, k\right)
\end{aligned}
$$

and

$$
\begin{aligned}
\bar{\gamma}_{A B}\left(z_{1}, z_{2}, k\right)= & \int d z^{\prime} p_{A}\left(z^{\prime}\right) \bar{\gamma}_{A M}\left(z_{1}, 2, k\right) \bar{\tau}_{A B}\left(z^{\prime}, z_{2}, k\right) \\
& +\int d z^{\prime} p_{A}\left(z^{\prime}\right) \bar{\tau}_{A A}(2,, 2, k) \bar{\tau}_{M}\left(z^{\prime}, z_{2}, k\right)
\end{aligned}
$$

There are several possible choices for an exact relation between the inhomogeneous density $\rho(z)$ and the correlation functions. We have chosen the one due to Wertheim and to Loveth, Mou, and Buff. The WLMB relation is

$$
\frac{\partial \ln \rho\left(z_{1}\right)}{\partial z_{1}}=-\frac{\partial \beta w\left(z_{1}\right)}{\partial z_{1}}+\int_{0}^{\infty} d z^{\prime} \frac{\partial \rho(z)}{\partial z^{\prime}} \bar{c}\left(z_{1}, z^{\prime}, 0\right)
$$

For mixtures $(A+B / W), W L M B$ assumes the form:

$$
\frac{\partial \ln \rho_{A}\left(z_{1}\right)}{\partial z_{1}}=-\frac{\partial \beta w_{A}\left(z_{1}\right)}{\partial z_{1}}+\tilde{\int} d z^{\prime} \frac{\partial \rho_{A}\left(z^{\prime}\right)}{\partial z^{\prime}} \tau_{M}\left(z_{1}, z^{\prime}, 0\right)
$$

and

$$
\frac{\partial \ln p_{B}\left(z_{1}\right)}{\partial z_{1}}=-\frac{\partial \beta w_{B}\left(z_{1}\right)}{\partial z_{1}}+\int d z^{\prime} \frac{\partial p_{A}\left(z^{\prime}\right)}{\partial z^{\prime}} \bar{\tau}_{A B}\left(z_{1}, z^{\prime}, 0\right)
$$

In our numerical calculations it has proven advantageous to rewrite the WLMB relation in terms of the cavity function $y_{A W}$ where $g_{A W}=h_{A W}+1=y_{A W} e_{A W}$ and $e_{A W}=\exp \left[-w_{A} / k T\right]$ so that equations (9.9) and $(9.10)$ become

$$
\begin{aligned}
\frac{\partial y_{A W}\left(z_{1}\right)}{\partial z_{1}}= & y_{A W}\left(z_{1}\right) \int_{0}^{\infty} d z^{\prime} \frac{\partial y_{A W}\left(z^{\prime}\right)}{\partial z^{\prime}} e_{A W} \bar{C}_{M}\left(z_{1}, z^{\prime}, 0\right) \\
& +y_{A W}\left(z_{1}\right) \int_{0}^{\infty} d z^{\prime} y_{A W} \frac{\partial e_{A W}\left(z^{\prime}\right)}{\partial z^{\prime}} \tau_{M}\left(z, z^{\prime}, 0\right)
\end{aligned}
$$

and

$$
\frac{\partial y_{B W}\left(z_{1}\right)}{\partial z_{1}}=y_{B W^{\prime}}\left(z_{1}\right) \int_{0}^{\infty} d z^{\prime} \frac{\partial y_{A W}\left(z^{\prime}\right)}{\partial z^{\prime}} e_{A W} \bar{c}_{A B}\left(z, z^{\prime}, 0\right)
$$




$$
+y_{B W}\left(z_{1}\right) \iint^{\prime} z^{\prime} y_{A W} \frac{\partial e_{A W}\left(z^{\prime}\right)}{\partial z^{\prime}} \bar{c}_{A^{\prime}}\left(z, z^{\prime}, 0\right)
$$

For the approximate closure relation (to oblain $c$ from $\gamma$ or $h$ and $u$ ) we chose 10 use the Percus. Yevick closure.

$$
c(r)=[1+\chi(r)][\exp (u(r) / k T)-1]
$$

Tests on Lennard-Jones fluids indicate that this choice is quite accurate.

The set of equations (9.6), (9.7), (9.11), (9.12), and (9.13) for mixtures is solved by the method of successive substitutions or Picard iterations. The approach, then, is 10 make an initial guess of the density profile and the pair correlation functions $\gamma_{i}$ to transform $\gamma$ to Fourier space $\bar{\gamma}_{i}$ to solve the inhomogene. ous $\mathrm{OZ}$ equations (9.6) and (9.7) for $\varepsilon$; to transform back to real space; to use the closure relation (9.13) to obtain a new estimate of $c$; to iterate (9.6), (9.7), and (9.13) to convergence; then, to substi. tute $\bar{c}$ in $(9.11)$ and $(9.12)$ oblaining a new estimate of $\rho\left(z_{1}\right)$. The criterion for convergence of each iteration loop is that the value of the $n^{\text {th }}$ iteration of the function shall differ at no point from the value of the $n-1^{n}$ iteration by no more than $10^{-6}$. Generally, correlation functions and density profles from a previously converged case were used as initial estimates. For startup, low density limits of the correla. tion functions were used (essentially, the Bolumann factors for $h$ and $c$, as well as $\rho(z)$ ).

For numerical solution we discretize the threc-dimensional equations (9.6) and (9.7) for $\bar{\gamma}$ and $\bar{\tau}$ such that there are $L, M$, and $N$ grids of $\Delta z_{1}, \Delta z_{2}$ and $\Delta k$ spacing, respectively.

$$
\begin{aligned}
& \bar{\gamma}(l, m, n)=\bar{\gamma}\left(l \Delta_{2}, m \Delta_{2}, n \Delta k\right) \\
& \quad l=1,2, \cdots, L_{i} \quad m=1,2, \cdots, M ; n=1,2, \cdots N
\end{aligned}
$$

Near the critical point of the solvent, the correlation functions $\gamma$ become long-ranged. In early work, we used $M>80$ with $\Delta z_{2}=0.03 \sigma, L=80$ with $\Delta z_{1}=0.05 \sigma$, and $N=50$ with $\Delta k=0.08(L=M)$. In order to conserve the use of memory and because both functions of the pairs $\left.C_{M}, \bar{C}_{M}\right)$ and $\left(C_{A B}, \bar{C}_{A B}\right)$ are not required at the same time, these function pairs share the same memory locations (via the EQUIVALENCE statement in FORTRAN). Thus, only six large arrays- $\gamma_{M}, \bar{\gamma}_{M}, \gamma_{A B}, \bar{\gamma}_{A B}, c_{A A}$, and $C_{A B}$-are required.

We have accurately calculated and tabulated the Hankel transform for $J_{0}$ from 0 to several hundred, the forward and reverse transformations agreeing to the ninth place.

The bulk fluid molecules were modeled to interact with the Lennard-Jones (LJ) 12:6potential, simulat. ing argon and methane. The wall-fluid potential used is the $L J$ 9:3 potential.

$$
w(2)=p \varepsilon\left[\frac{2}{15}\left[\frac{m \sigma}{2}\right)^{9}-\left(\frac{m \sigma}{2}\right)^{3}\right]
$$

where $p$ and $m$ are numerical factors to modify the bulk fluid force constants $\varepsilon$ and $\sigma$.

\subsection{TEST CALCULATIONS}

The first lest we performed was reproducing one of the cases by Plischke and Henderson (9.6) for a pure $\mathrm{L}$ fluid in contact with a hard wall. For the state $T^{\circ}=k T / \varepsilon=1.35$ and $p^{\circ}=p \sigma^{3}=0.40$ our program reproduced Plischke and Henderson's density profile quantitatively. In all of the calculations reported below, we have used finer grid spacing and longer range in the correlation functions than those used by Plischke and Henderson.

The range of the pair correlation function grows without limit upon approach to the CP. This is a significant issue because the value of $f(r)$ in equation (9.3) must be 0 at the maximum value of $r$ to perform the Hankel transform. We have tested the effects of the range of the numerical calculations for 
the parameters shown in Table 9.1.

Table 9.1 Numerical parameters for testing effect of range of calculations

\begin{tabular}{ccccccccc}
\hline \hline $\max Z_{1}$ & $\Delta Z_{1}$ & $N_{Z_{1}}$ & $\max Z_{2}$ & $\Delta Z_{2}$ & $N_{Z_{2}}$ & $\max R$ & $\Delta R$ & $N_{R}$ \\
\hline 6.0 & 0.05 & 120 & 6.0 & 0.05 & 120 & 4.0 & 0.08 & 50 \\
9.0 & 0.05 & 180 & 9.0 & 0.05 & 180 & 4.8 & 0.08 & 60 \\
9.5 & 0.05 & 190 & 9.5 & 0.05 & 190 & 4.8 & 0.08 & 60 \\
\hline
\end{tabular}

Figure 9.1 shows the effect of range on results for the wall-solvent pair correlation function, $g \mathrm{WA}(z)$, for a state $\left(T^{\circ}=1.35\right.$ and $\rho^{\circ}=0.35$ ) close to the critical point. The maximum range used in earlier $(9.4)$ work $(120,120,50)$ is evidently inadequate for quantitative results. The agreement between results with the range used in the present calculations $(180,180,60)$ and those with still longer range $(190,190,60)$ indicates that the present results are the first quantitatively valid application of the HPS approach in the PY approximation.

One of the failings of the simpler HAB (9.3) approach is the inability to produce the pre-wetting transition that is exhibited by density functional theory and Monte Carlo simulations. The more accurate HPS (9.6) approach has not previously been tested for this capability. We have attempted to test this question, but, unfortunately, the tests have not been conclusive. The calculations are difficult because at the transition the range becomes unbounded; nevertheless, we attempted to observe signs of the onset of the transition. An additional difficulty with the calculations results from the fact that the location of the prewelting transition, $?^{\circ}, \rho^{* m}$, is very close to the bulk vapor-liquid phase boundary. Indeed, it was only by careful attention to this detail, that Finn and Monson (9.7) were able to first observe the transition in simulations after earlier failures by others. Figure 9.2a shows their result clearly demonstrating the prewetting transition with increasing bulk density. We were hindered in the present calculations by the fact that the location of the bulk vapor-liquid phase boundary for the L-J fluid in the PY approximation is not accurately known. Despite the difficulties and imperfections of the tesh we show results in Figure $9.2 \mathrm{~b}$ that are quite suggestive of the onset of a possible prewetting transition; note, in particular, the appearance of shoulders representing second and third adsorbed layers.

We have drawn the following conclusions from lesting the HPS (9.6) integral equation approach to studying adsorption equilibrium: - Although we have seen suggestions of a prewetting transition, we have been unsuccessful so far in demonstrating that the HPS theory can exhibit this phenomenon. - We have presented the first quantitative results with the HPS approach, using finer grids and longer range than previous calculations and presenting daunting computer memory requirements.

\subsection{ADSORPTION FROM SCF}

We have studied several cases of adsorption from SCF solution. We have studied adsorption of the solvent (A) and the attractive solute (B) on a hard wall (HW) for temperatures approaching the bulk CP from above. These results are presented in Section 9.3.1. We have studied adsorption of the solvent (A) and both the atractive solute (B) and the repulsive solute (also B) on a $\mathrm{LJ}$ wall for a near critical state. These results are presented in Section 9.3.2. Table 9.2 presents the parameters of the interaction potentials used in this work. With the HW, the solvent (A) parameters model argon; the attractive and repulsive solutes (B) have the same size as $A$ with an arbitrarily greater or smaller interaction encrgy parameter. With the $\mathrm{LJ}$ wall, again the solvent (A) parameters model argon; the solvent-wall parameters model argon with solid $\mathrm{CO}_{2}$; the attractive solute (B) models methane; and the repulsive solute (also $\mathrm{B}$ ) has the same size as the methane model but has interaction energy proportionately smaller than that of argon as methane is larger. 
Table 9.2. Parameters of interaction potentials used

\begin{tabular}{lllll}
\hline \hline & pair & $\varepsilon / k(\mathrm{~K})$ & $\sigma(\mathrm{A})$ & material approximated \\
\hline Hard Wall & & & & \\
& A-A & 119.80 & 3.400 & argon-argon \\
& A-B(repulsive) & 100.24 & 3.400 & argon-repulsive solute \\
& A-B(aturactive) & 143.16 & 3.400 & argon-attractive solute \\
\hline W:9-3 Wall & & & & \\
& A-A & 119.80 & 3.400 & argon-argon \\
& A-B(repulsive) & 107.82 & 3.606 & argon-repulsive solute \\
& A-B(aturactive) & 134.45 & 3.606 & argon-methane solute \\
& W-A & 153.00 & 3.727 & solid $\mathrm{CO}_{2}$-argon \\
& W-B(repulsive) & 297.69 & 3.957 & solid $\mathrm{CO}_{2}$-repulsive solute \\
& W-B(attractive) & 297.69 & 3.957 & solid $\mathrm{CO}_{2}$-methane solute \\
& & & &
\end{tabular}

9.3.1 Adsorption at a Hard Wall. Figures 9.3a and 9.3b show density profiles near a HW for a SCF LJ solvent and an infinitely dilute, repulsive $L J$ solute, respectively, for density $\rho^{\circ}=0.40$ and temperatures, $T^{*}=5.00,3.00,2.00$, and 1.35. For the HW system, the $z=0$ intercept of the solvent-wall density profile is a measure of the bulk fluid pressure $P^{*}=P \sigma^{3} / \varepsilon=g(0) p^{*} T^{*}$. Of course, the pressure at constant density increases with increasing temperature. It is interesting to note, however that, for the solvent, there is a net deficit at the wall at the near-critical state and for the attractive solute the deficit is substantially greater. The near-critical solvent has a higher affinity for the attractive solute (and even for other solvent molecules) than does the HW.

9.3.2 Adsorption at a Lennard-Jones Wall. Figures 9.4a, 9.4b, and 9.4c show the effect of the wall strength on the density profiles near an attractive $L J: 9-3$ wall (the HW profiles are also shown for comparison) for the state $\dot{\rho}^{*}=0.35$ and $T^{*}=1.35$ for the $\mathrm{LJ}$ solvent, the repulsive solute, and the attractive solute, respectively. The parameter shown for each of the profiles is the percent of the full LJ:9-3 potential used by Finn and Monson (9.7) to model argon adsorption on solid carbon dioxide. It is evident that compared to the HW, the $\mathrm{LJ}: 9.3$ wall attracts considerable adsorption, the degree of adsorption increasing with the strength of the wall. Accompanying the increased adsorption is an increase in the structure near the wall, showing clear first and second, and even third, maxima in the profile.

Figures $9.5 \mathrm{a}, 9.5 \mathrm{~b}$, and $9.5 \mathrm{c}$ replot some of the previous results in such a way as to emphasize the relative adsorption of the solvent, the repulsive solute, and the attractive solute as the strength of the wall interaction increases. The SCF solvent draws the attractive solute into solution and away from the wall so that its adsorption on the HW is less than that of the solvent. In contrast, the repulsive solute is expelled from the SCF solvent so that its adsorption on the HW is relatively greater than that of the solvent.

The LJ:9-3 wall with 50\% of the wall strength of the Finn-Monson (9.7) system attracts the solvent, attractive solute, and repulsive solute so that the adsorption of all three is increased. But the adsorption of the attractive solute has increased most so that it has approximately equaled that of the solvent. Here we see competition between the SCF solvent and the wall for the attractive solute. At 75\% of the FinnMonson wall strength the adsorption of the attractive solute has overtaken that of the solvent and approaches that of the repulsive solute.

In figure 9.6a we examine the radial distribution for the solute-solvent correlation $h_{A B}(r)$ for different values of 2 , the distance from the wall for the attractive solute at the state $\rho^{*}=0.35$ and $T^{*}=1.35$. The wall-particle profile shown in figure $9.6 \mathrm{~b}$ gives a measure of the relative fluid density at the various distances $z$ from the wall. At $z=0.0 \sigma, h_{A B}(r)$ shows a single broad peak dropping monotonically to the bulk value; such structure is similar to that of a low density gas. Note in Figure $9.6 \mathrm{~b}$ that the density at $z=0.00$ is low. At $z=1.0 \sigma$, the structure looks more like that of a liquid with a distinct second 
maximum. In Figure 9.6b, one can see the correspondingly high density at this distance from the wall. The variation in the structure with distance from the wall is qualitatively similar to that seen by Plischke and Henderson (9.0.

\subsection{DISCUSSIONS}

Ours are the first attempts, to our knowledge, to undertake a molecular-based study (using the inhomogencous Ornstein-Zemike equation) of adsorption equilibrium between a solution (mixture of $A$ and $B$ ) and a surface $(W)$ and the first time such study has been focused on SCF solutions. This extension of the integral equation method of Plischke. Henderson, and Sokolowski (4-6) has presented significant challenge, and study of interesting problems has aggravated the large memory requirements. Approach to the bulk CP requires long range; approach to surface wetting also requires long range; strong (realistic) wall forces require fine grid spacing; and large solute-solvent asymmetry also requires fine grid spacing. The nested iterations of the inhomogeneous solvent-solvent and solute-solvent Ornstein-Zernike equations and the Wertheim-Lovett-Mou-Buff equations required long runs.

Because of these computational challenges, there was difficulty in performing calculations for states very close to the $\mathrm{CP}$ or very close to the prewetting transition; there was also difficulty in performing calculations with strong wall potentials. Even so, the range was longer and the grid finer than in previ. ous studies by this method.

Adsorption from SCF solutions on a realistic (but weak) wall exhibited expulsion of a repulsive solute from SCF solvent to the wall and competition for an attractive solute between the solvent and the wall.

\section{References}

Attard, P. J. Chem. Phys., 1989, 3072.

Attard, P. J. Chem. Phys., 1989, 3083.

R. J. Baxter, "Method of Solution of the Percus-Yevick, Hypernetted-Chain, or Similar Equations," Phys. Rev., 154, 170 (1967).

R. J. Baxter, "Omstein-Zemike Relation for a Disordered Fluid," Aust. J. Phys., 21, 563 (1968).

R. J. Baxter, "Ornstein-Zernike Relation and Percus-Yevick Approximation for Fluid Mixtures," J. Chem. Phys., 52, 4559 (1970).

J. F. Brennecke and C. A. Eckert, "Molecular Interactions from Fluorescence Spectroscopy," Proc. Int. Symp. on Supercritical Fluids, M. Perrut, Ed., 263, Nice, France (1988).

J. F. Brennecke and C. A. Eckerh, "Fluorescence Spectroscopy Studies of Intermolecular Interactions in Supercritical Fluids," in Supercritical Fluid Science and Technology, K. P. Johnston and J. M. L. Penninger, Eds., ACS Symp. Ser. No, 406, Ch. 2, in press (1989).

J. J. Brey and A. Santos, "On the Critical Behavior of the Percus-Yevick Equation for Nontruncated Potentials," J. Chem. Phys., 82, 4312 (1985).

F.V. Bright, T.A. Betts, and J. Zagrobelny, Chap.4, in ACS Symposium Series \#488, Recent Advances in Supercritical Fluid Technology: Theoretical and Applied Approaches in Analytical Chemistry, (American Chemical Soc. Washington D.C 1992) pp.48-59.

A. Busigin and C.R. Phillips, Mol. Phys. 76, 89 (1992).

C. Carlier and T.W. Randolph, AIChE J 1992.

A.A. Chialvo, J.Phys.Chem. 97, 2740 (1993). 
A.A. Chialvo and P.T. Cummings, Preprint (1993).

H. D. Cochran and L. L. Lee, "Solvation Structure in Supercritical Fluid Mixtures based on Molecular Distribution Functions," in Supercritical Fluid Science and Technology. K. P. Johnston and J. M. L. Penninger, Eds., ACS Symp. Ser. 406, Ch. 3, in press (1989).

H.D. Cochran, L.L. Lee, and D.M. Pfund, Fluid Phase Equil. 34, 219 (1987).

H. D. Cochran, L. L. Lee, and D. M. Pfund, "Application of the Kirkwood-Buff Theory of Solutions to Dilute Supercritical Mixtures," Fl. Ph. Equilib., 34, 219 (1987).

H. D. Cochran, L. L. Lee, and D. M. Pfund, "Study of Fluctuations in Supercritical Solutions by an Integral Equation Method," Proc. Int. Symp. on Supercritical Fluids, M. Perrut, Ed., Nice, France, 245 (1988).

H. D. Cochran, L. L. Lee, and D. M. Pfund, "Structure and Properties of Supercritical Fluid Mixtures from Kirkwood-Buff Fluctuation Theory and Integral Equation Methods," submitued to Fluctuation Theory of Mixtures, E. Matueoli, Ed., Adv. in Thermo. Ser., Taylor and Francis (1989).

H. D. Cochran, D. M. Pfund, and L. L. Lee, "Theoretical Models of Thermodynamic Properties of Supercritical Solutions," Sep. Sci \& Tech., 23, 2031 (1988).

N. A. Collins, P. G. Debenedelti, and S. Sundaresan, "Disproportionation of Tolvene over SZM-5 under Near-Critical Conditions," AlChE J., 34, 1121 (1988).

P. T. Cummings and P. A. Monson, "A New Method for the Numerical Solution of Integral equation approximations," presented at 10th Symp. on Thermophys. Props., Gaithersburg, MD, June 20-23 (1988).

P. G. Debenedetti, "Clustering in Dilute, Binary Supercritical Mixtures: A Fluctuation Analysis," Chem. Eng. Sci., 42, 2203 (1987).

Debenedetti, P. G.; Mohamed, R. S. J. Chem. Phys., 1989, 90, 4528.

P. G. Debenedetti and R. S. Mohamed, "Altractive, Weakly Altractive, and Repulsive Near-Critical Systems," J Chem. Phys., 90, 4528 (1989).

Ebner, C; Saam, W. F. Phys. Rev. Lell., 1977, 38, 1486.

C. A . Eckerh, D. H. Ziger, K. P. Johnsion, and T. K. Ellison, "The Use of Partial Molar Volume Data to Evaluate Equations of State for Supercritical Fluid Mixtures," Fl. Ph. Equilib., 14, 167 (1983).

C. A. Eckert, D. H. Ziger, K. P. Johnston, and S. Kim, "Solute Partial Molar Volumes in Supercritical Fluids," J. Phys. Chem., 90, 2798

(1986).

E. Enciso, Mol. Phys. 56, 129 (1985).

Evans, R.; Tarazona, P.; Marconi, U. M. B. Mol. Phys., 1983, 50, 993.

Finn, J. E.; Monson, P. A. Phys. Rev. A, 1989, 39, 6402.

Finn, J. E.; Monson, P. A. Mol. Phys., 1991, 72, 661.

M. J. Gillan, "A New Method of Solving the Liquid Structure Integral Equations," Mol. Phys., 38, 1781 (1979).

M. Gitterman and I. Procaccia, "Quantitative Theory of Solubility in Supercritical Fluids," J. Chem. Phys., 78, 2648 (1983).

J. B. Hannay and J. Hogarth, "On the Solubility of Solids in Gases," Proc. Roy. Soc. London, A29, 324 (1879).

J. B. Hannay and J. Hogarth, "On the Solubility of Solids in Gases," Proc. Roy, Soc. London, A30, 484 (1880).

Henderson, D.; Abraham, F. F.; Barker, J. A. Mol. Phys., 1976, 31, 1291. 
K. P. Johnston, W. Flarsheim, B. Hmjez, A. Mehta, M. Fox, and A. Bard, "Solvent Effect on Chemical Reactions at Supercritical Fluid Conditions," Proc. Int. Symp. on Supercritical Fluids. M. Perrut, Ed., 907. Nice, France (1988).

K. P. Johnston, S. Kim, and J. Combs, "Spectroscopic Determination of Solvent Strength and Structure in Supercritical Fluid Mixtures, A Review," in Supercritical Fluid Science and Technology, K. P. Johnston and M. L. Penninger, Eds., ACS Symp. Ser. 406, Ch. 5, in press (1989).

O. Kajimoto M. Futakami, T. Kobayashi, and K. Yamasakd, "Charge-Transfer-State Formation in Supercritical Fluid: (N.N.Dimethylamino)benzonitrile in SCF sub 3 HS," J. Phys. Chem., 92, 1347.1352 (1988).

S. Kim and K. P. Johnston, "Molecular Interactions in Dilute Supercritical Fluid Solutions," I\&EC Res., 26. 1206 (1987).

S. Kim and K. P. Johnston, "Clustering in Supercritical Fluid Mixtures," AIChE J., 33, 1603.1611 (1987).

J. G. Kirkwood and F. P. Buff, "The Statistical Mechanical Theory of Solutions. 1," J. Chem. Phys., 19, 774 (1951).

Kjellander, R.: Sarman, S. Mol. Phys., 1991, 665.

S. Kim and K. P. Johnston, "Molecular Interactions in Dilute Supercritical Fluid Solutions," I\&EC Res., 26, 1206-1213 (1987).

B.L. Knutson, D.L. Tomasko, C.A. Ecker, P. O. Debenedett, and A.A. Chialvo, Chap.S, in ACS Symposium Series 4488, Recent Advances in Supercritical Fluid Technology: Theoretical and Applied Approaches in Analytical Chemisiry, (American Chemical Soc. Washington D.C 1992) pp.60-72.

S. Labik, A. Malijersky, and P. Vonka, Ibid. (1985).

S. Labik, A. Malijevsky, and P. Vonka, Mol. Phys. 56, 709 (1985).

S. Labik, A. Malijevsky, and P. Vonka, "A Rapidly Convergent Method of Solving the Oz Equation," Mol. Phys., S6, 709 (1985).

F. Lado, "Perturbation Correction for the Free Energy and Structure of Simple Fluids," Phys. Rev. A, 87, 2548 (1973).

L. L. Lee, Molecular Thermodynamics for Nonideal Fluids, Butterworths, Boston (1988). Also, J.S. Rowlinson and F.L. Swinton, "Liquids and Liquid Mixtures", (Butterworths, Boston, 1982), p.254.

Lee, L. L. Molecular Thermodynamics of Nonideal Fluids; Butlerworths: Stoncham, MA, 1988; 164. 175.

Lee, L.L.; Debenedetti, P.G.; Cochran, H.D. Flucluation theory of supercritical solutions; in Supercritical Fluid Technology; Ely, J.F.; Bruno, TJ., Eds.; Chemical Rubber Co: 1991.

Loveu, R.; Mou, C. Y.; Buff, F. P. J. Chem. Phys., 1976, 65, 570.

J. J. Nicolas, K. E. Gubbins, W. B. Streeth and D. J. Tildesley, "Equation of State for the LennardJones Fluid," Mol. Phys., 37, 1429 (1979).

P.A. Monson, Mol. Phys. 47, 435 (1982).

Percus, J. K.; Yevick, G. J. Phys. Rev., 1958, 110. 1.

1. B. Petsche and P. G, Debenedetti, "Solute-Solvent Interactions in Infinitely Dilute Supercritical Mixtures: A Molecular Dynamics Investigation," J. Chem. Phys., submitted (1989).

Pelsche I.B.; Debencdetti, P.G. J. Phys. Chem. 1991, 95, 386.

D. M. Pfund, L. L. Lee, and H. D. Cochran, "Application of the Kirkwood-Buff Theory of Solutions to Dilute Supercritical Mixtures, Il. The Excluded Volume and Local Composition Models," Fl. Ph. Equilib., 39, 161 (1988). 
Piischke, M: Henderson, D. J. Chem. Phys., 1986, 84, 2846.

Plischke, M.: Henderson, D. Proc. Roy. Soc. London A, 1986, $404,323$.

Saam, W. F.; Ebner, C. Phys. Rev. A, 1978, 17, 1768.

G. M. Schneider, J. Eller, U. Harhaus, I. F. Holscher, O. Katzenskj-Ohling, A. Koppner, J. Kulka, D. Nickel, J. Rubesamen, and A. Wilsch, "Thermodynamic, Spectroscopic and Phase Equilibrium Investi. gations on Polar Fluid Mixtures at High Pressures," Pure \& Appl. Chem., 59, 1115.1126 (1987).

Sokolowski, S. J. Chem. Phys. 1980, 73, 3509.

B. Subramanian and M. A. McHugh, "Reactions in Supercritical Fluids - A Review," I\&EC Proc. Des. \& Dev., 25, 1 (1986).

R. O. Watts, "Pcrcus-Yevick Equation Applied to a Lennard-Jones Fluid," J. Chem. Phys., 48, 50 (1968).

R.O. Watts, J. Chem. Phys. 48, 50 (1968).

Wertheim, M. J. Chem. Phys., 1976, 65, 2377.

Wu, R. S. Ph.D. Dissertation, University of Oklahoma, 1990.

C. R. Yonker, S. L. Frye, D. R. Kalkwarf, and R. D. Smith, "Characterization of Supercritical Fluid Solvents Using Solvatochromic Shifts," J. Phys. Chem., 90, 3022.3026 (1986).

C. R. Yonker and R. D. Smith, "Solvatochromic Behavior of Binary Supercritical Fluids: The Carbon Dioxide/2-Propanol System," J. Phys. Chem., 92, 2374-2378 (1988). 


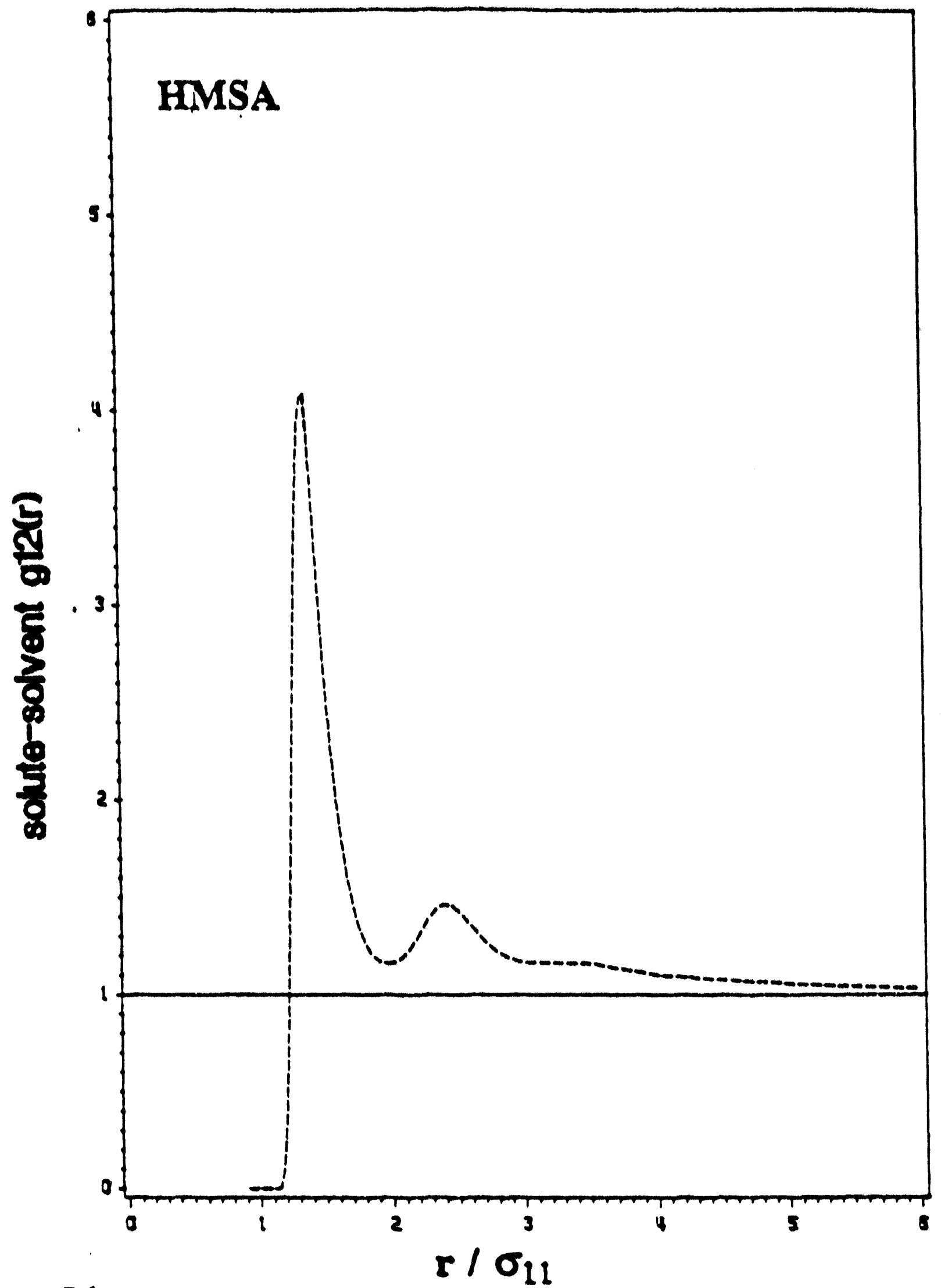

Figure 5.1 Solute-solvent pair correlation function at $T^{*}=1.4$ ani $\rho^{*}=0.35$. $\mathrm{Xe}$ in $\mathrm{Ne}$ (attractive mixture). HMSA closure, solut Cunntin- - in-a 


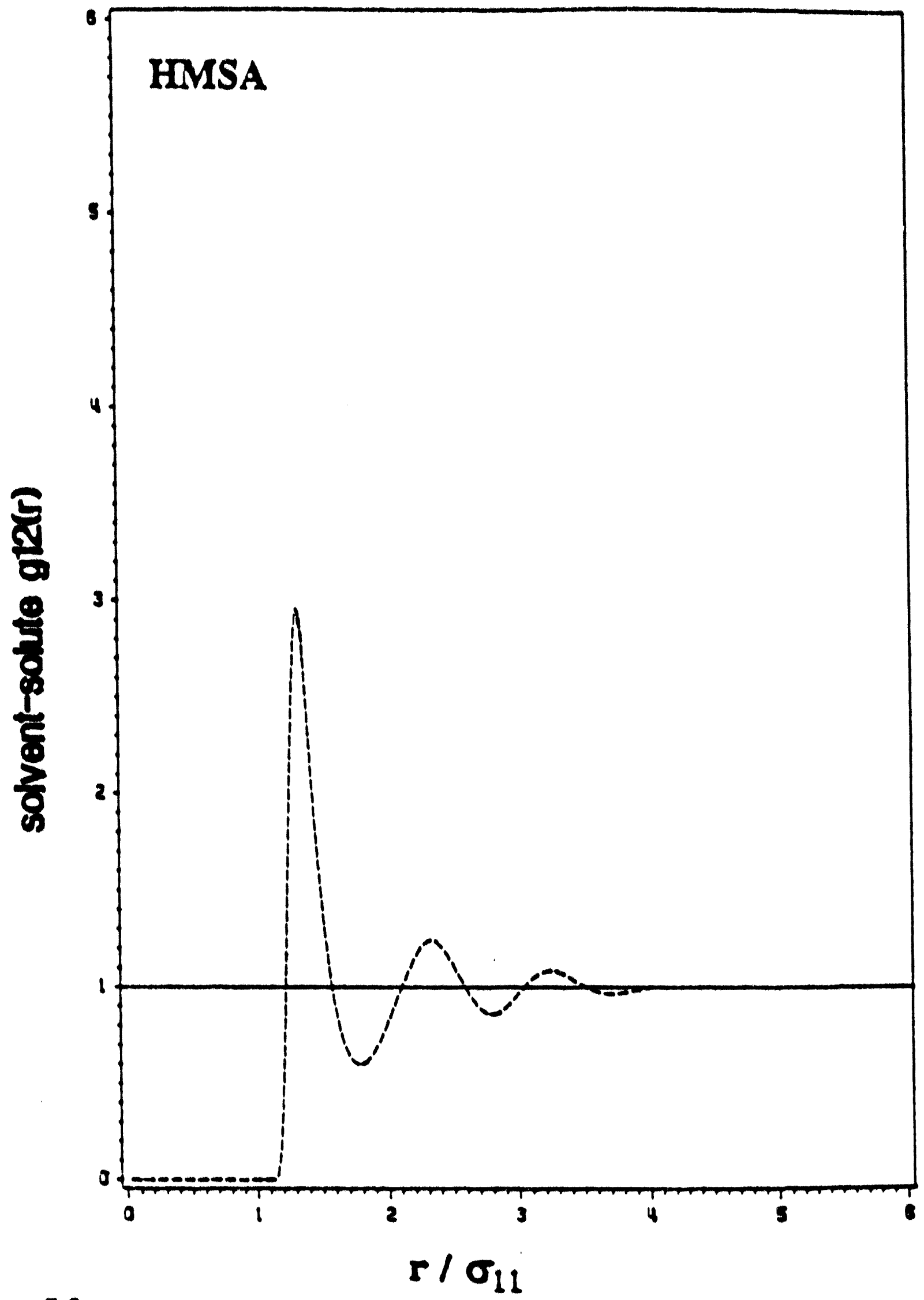

Figure 5.2 Solute-solvent correlation function at $T^{*}=1.4$ and $\rho^{*}$ $=0.8$. $\mathrm{Xe}$ in $\mathrm{Ne}$ (attractive mirture). HMSSA closure, solute fractior $=1 \mathrm{n} \rightarrow$ 


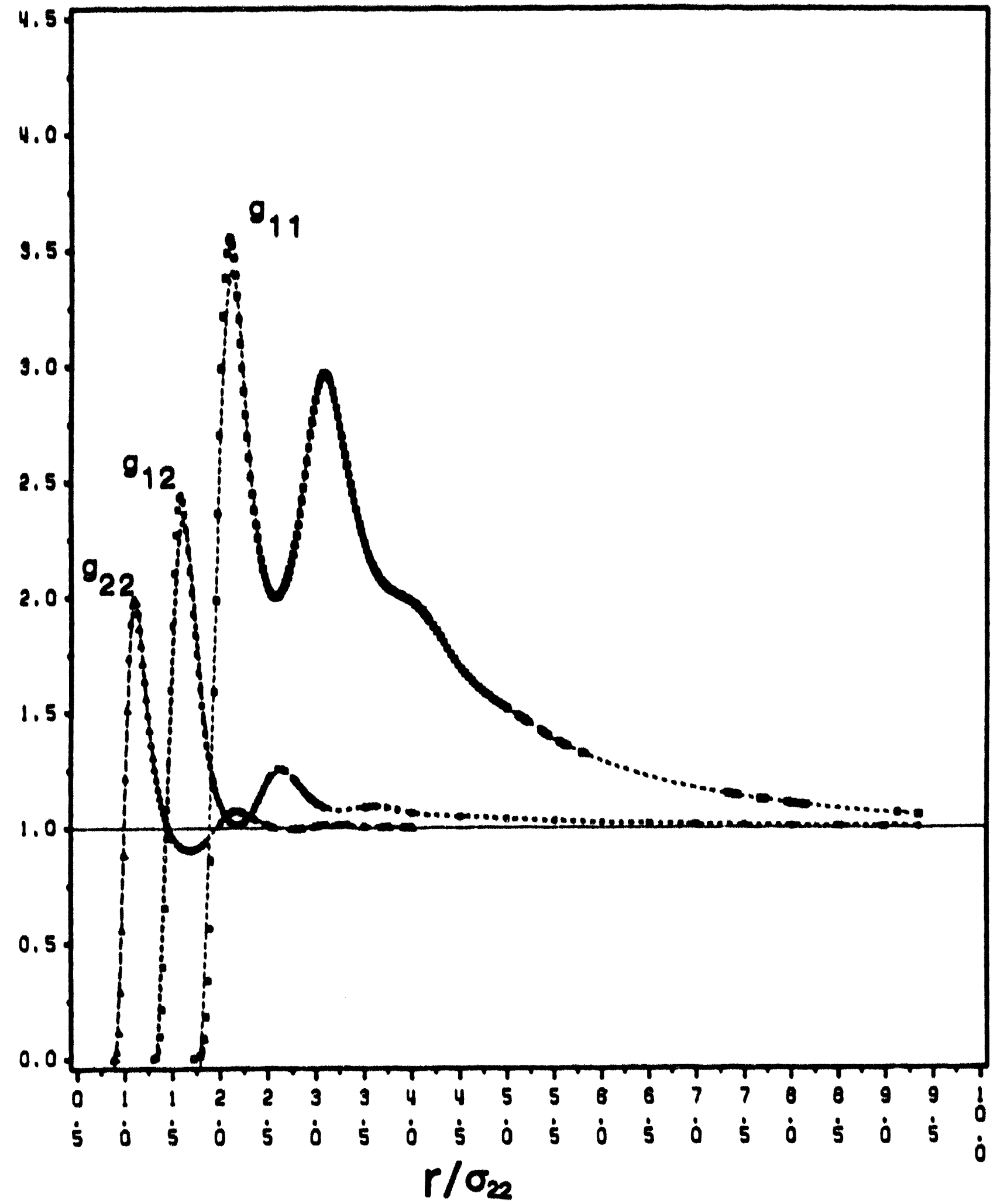

Figure 5.3 Pair correlation functions for solute-solute (squares), solute-solvent (stars), and solvent-solveat (triangles) interactions at $T^{*}=1.37, \rho^{*}=0.4248$. Potential parameters as per Table 3 (pyrene-cartion dioxide). RHNC closure. Solute mole fraction $=3 \times 10^{-1}$. (From Wu, R.-S., Lee, L. L., and Cochran, H. D., Ind. Eng. Chem. Res., 29, 977, 1990. With permission.) 


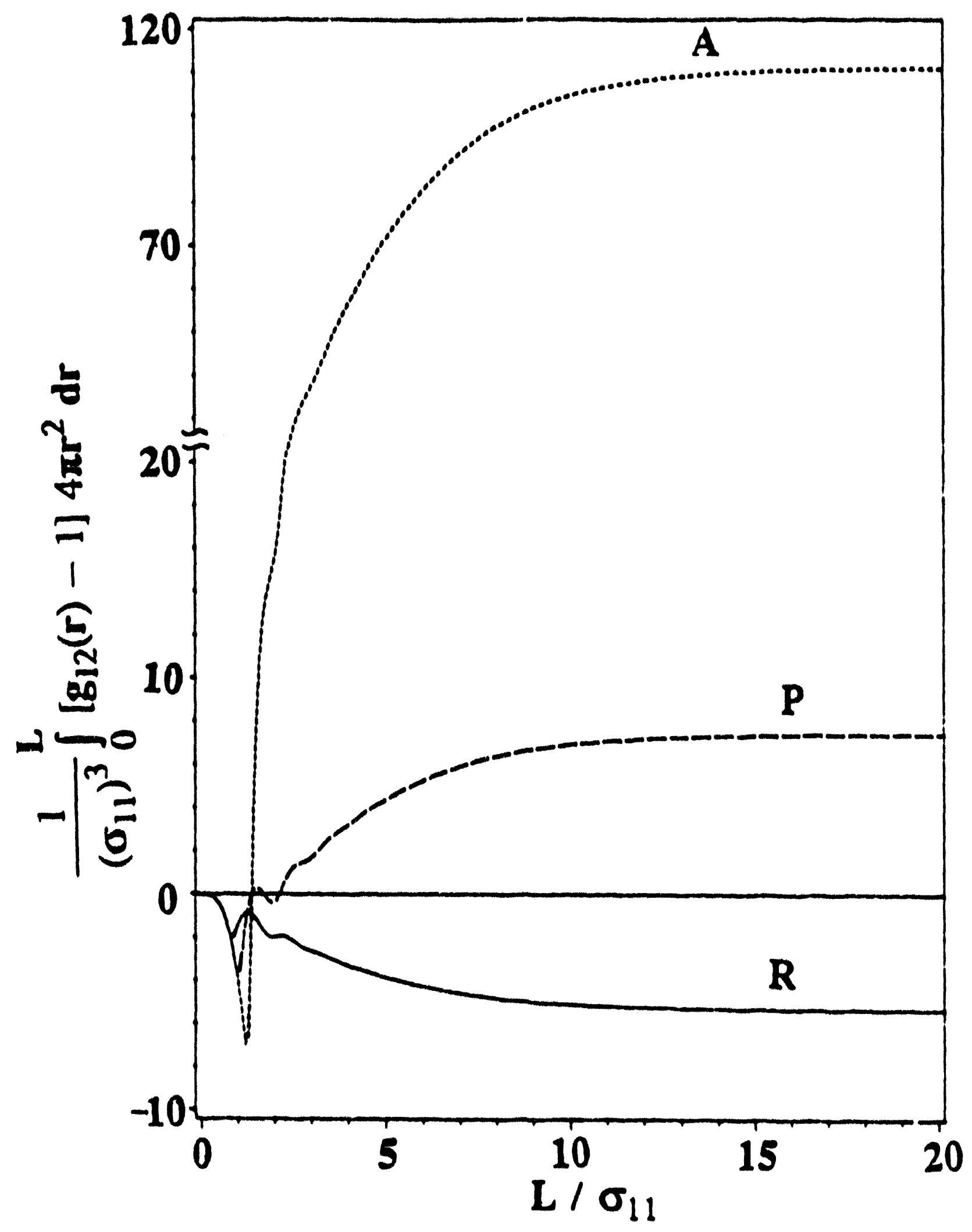

Figure 5.4 Number of excess solvent molecules within a sphere of radius $L$ around a central solute, for the pure Lennard-Jones solvent $(P)$, an attractive tnixture $\left(A ; X_{e}\right.$ in $\mathrm{Ne}$ ), and a repulsive mixture (R; Ne in $X e)$, at $T^{*}=1.4, p^{*}=0.35$. Potential parameters as per Table 4 ( $A=$ Mixture 1; $B=$ Mixture 2). PY closure. Solute mole fraction $=10^{-9}$. (From Wu, R..S., Lee, L. L., and Cochran, H. D., Ind. Eng. Chem. Res. 29. 977. 1990. With Dermission.) 


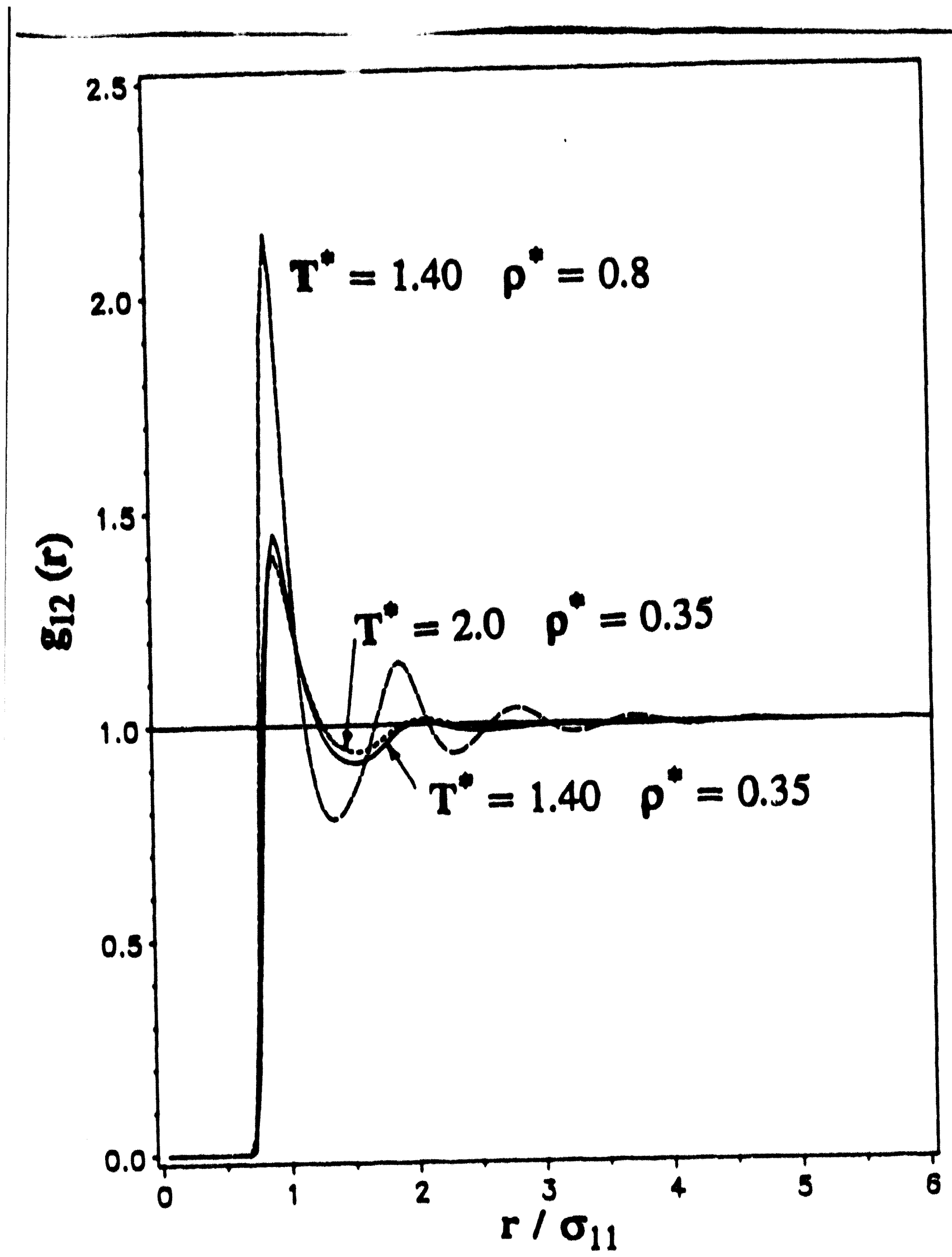

Figure 5.5(a)Solute-solvent pair correlation functions at three different state conditions for dilute $\mathrm{Ne}$ in $\mathrm{Xe}$ (repulsive mixture). Potential parameters as per Table 4 (mixture 2). PY closure. Solute mole fraction $=10^{-9}$. (From Wu, R.-S., Lee, L. L., and Cochran, H. D. Ind. Eng. Chem. Res., 29, 977, 1990. ᄃ $\because ;$ With permission.) 


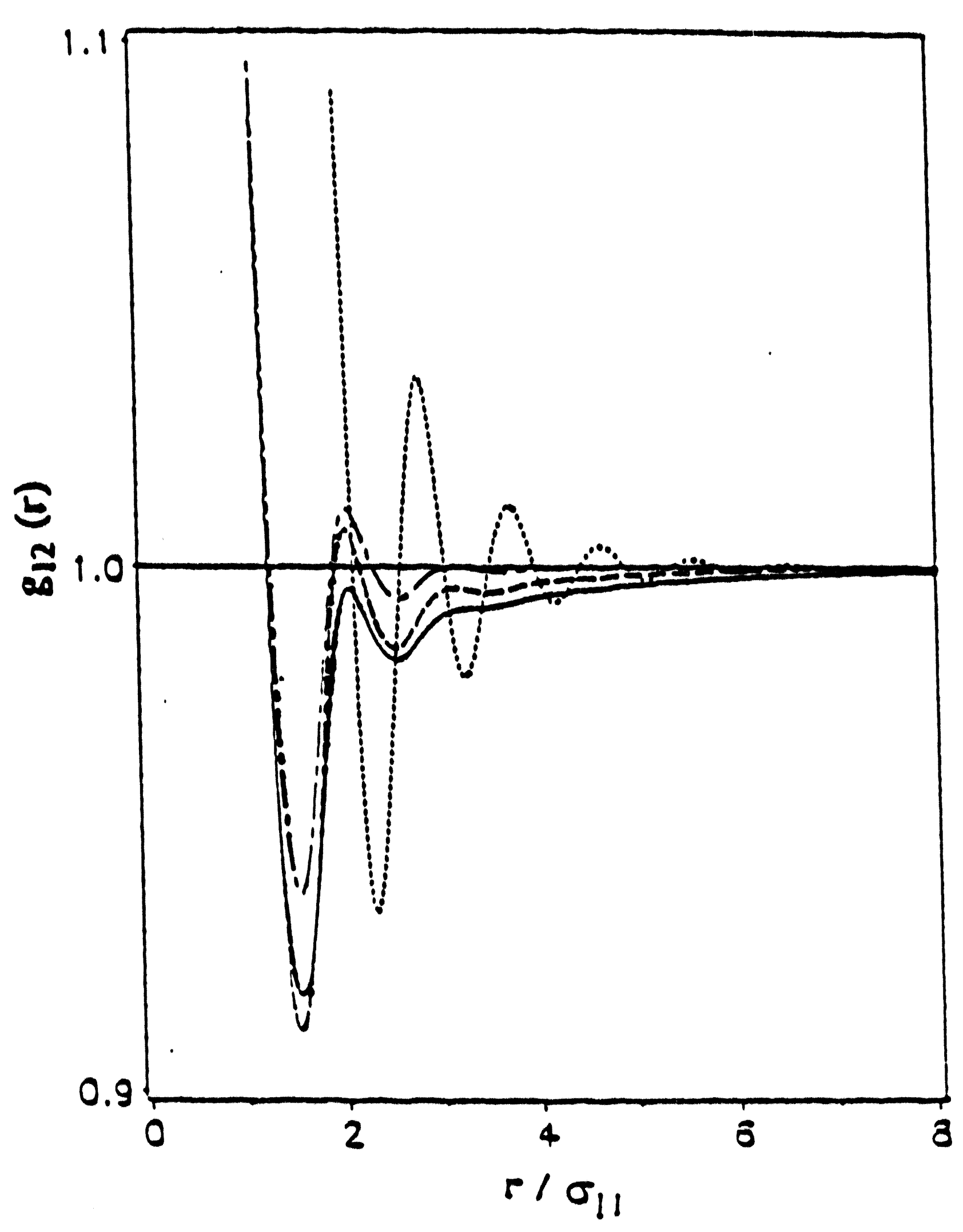

Figure 5.5 (b) 
Ind. Eng. Chem. Res., Vol. 29, No. 6, 1990983

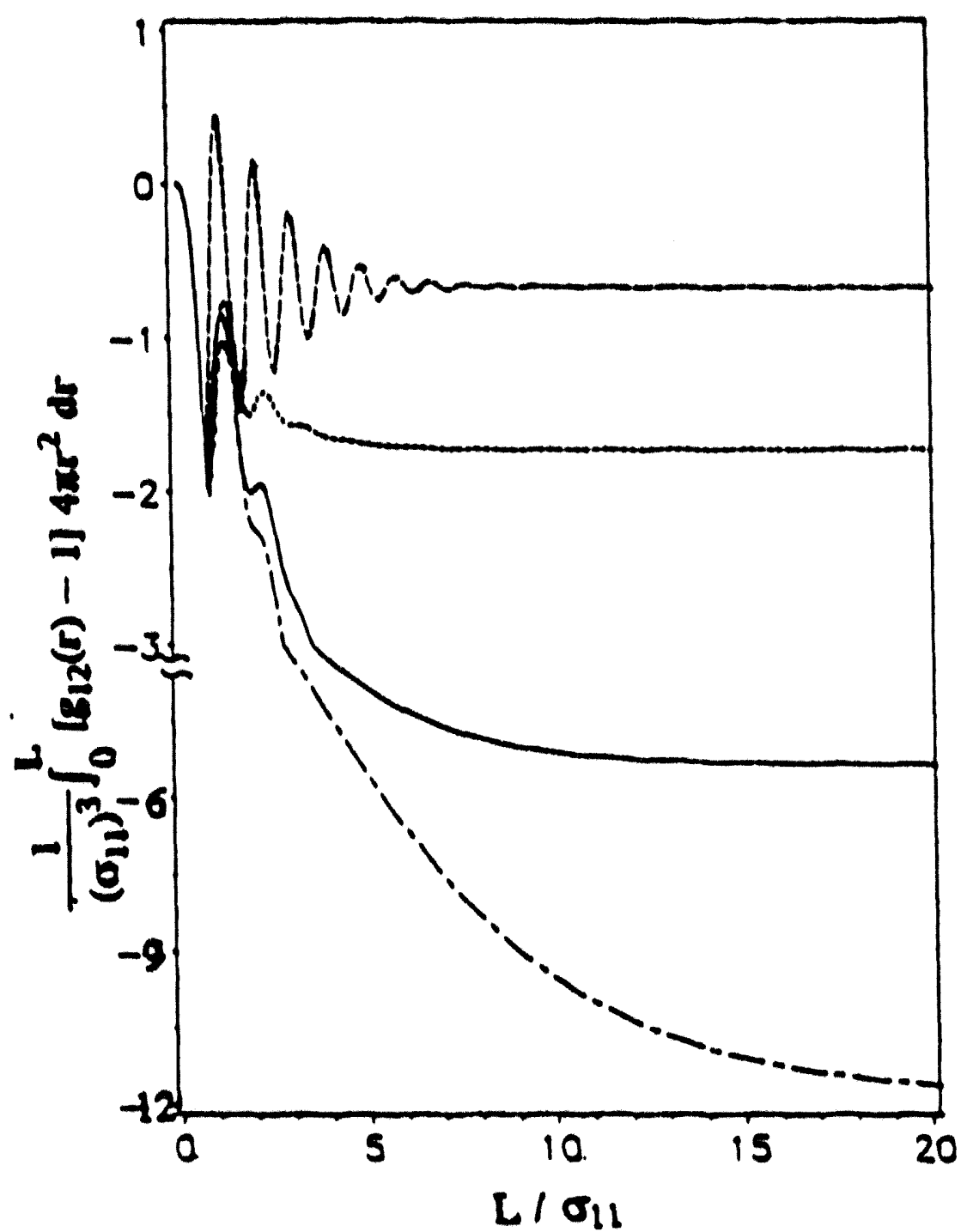

Figure 5.6 Number of excess solvent molecules within a sphere of radius $L$ around a solute, at $T^{*}=1.34, \rho^{*}=0.27(---) ; T^{*}=1.4$, $\rho^{*}=0.8(--) ; T=1.4, \rho^{*}=0.35(-) ;$ and $T *=2.0, \rho^{*}=0.35(-$. for dilute $\mathrm{Ne}$ in $\mathrm{Xe}$ (repulsive mirture). PY closure. Solute mole fraction $=10^{-5}$. 


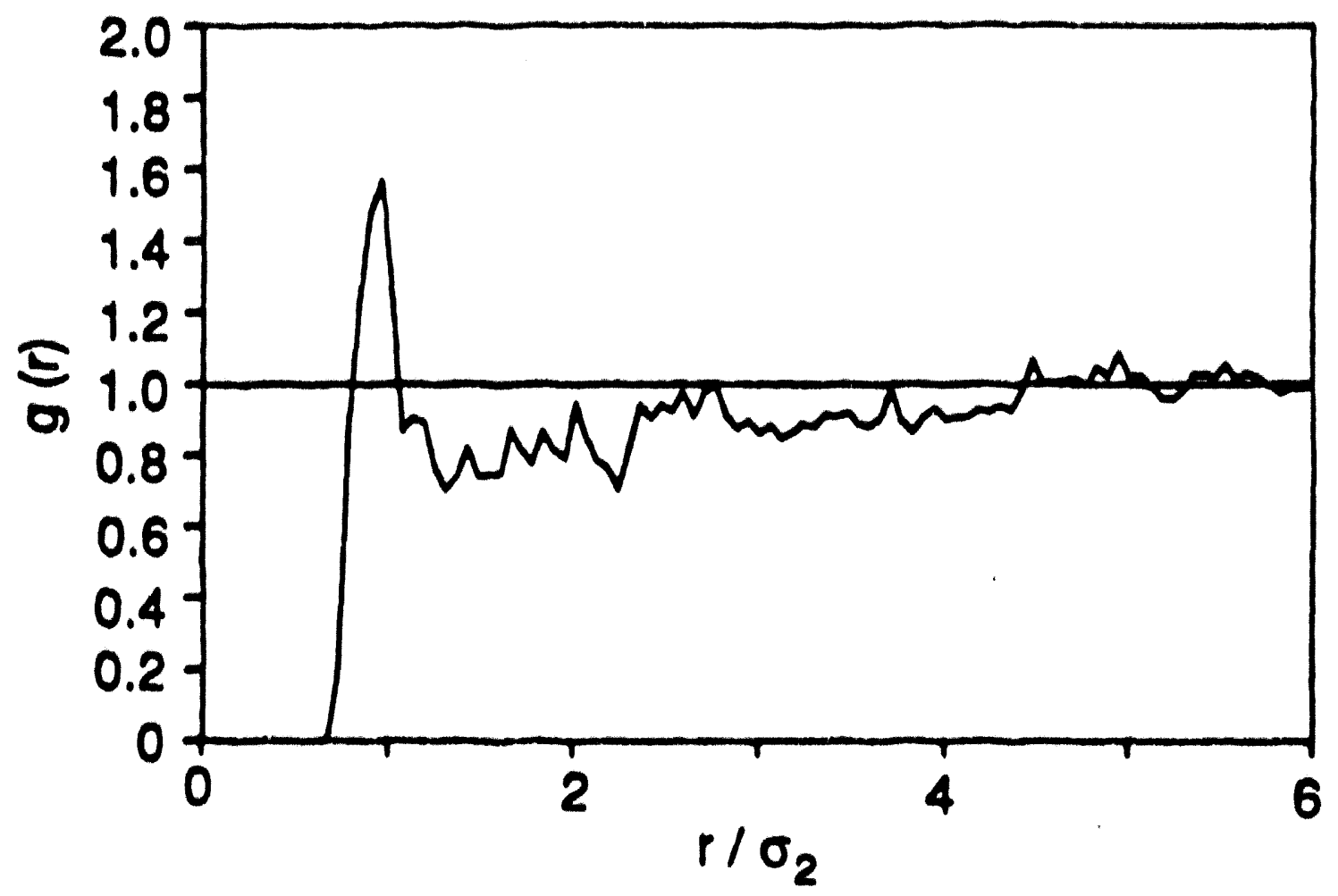

(a)

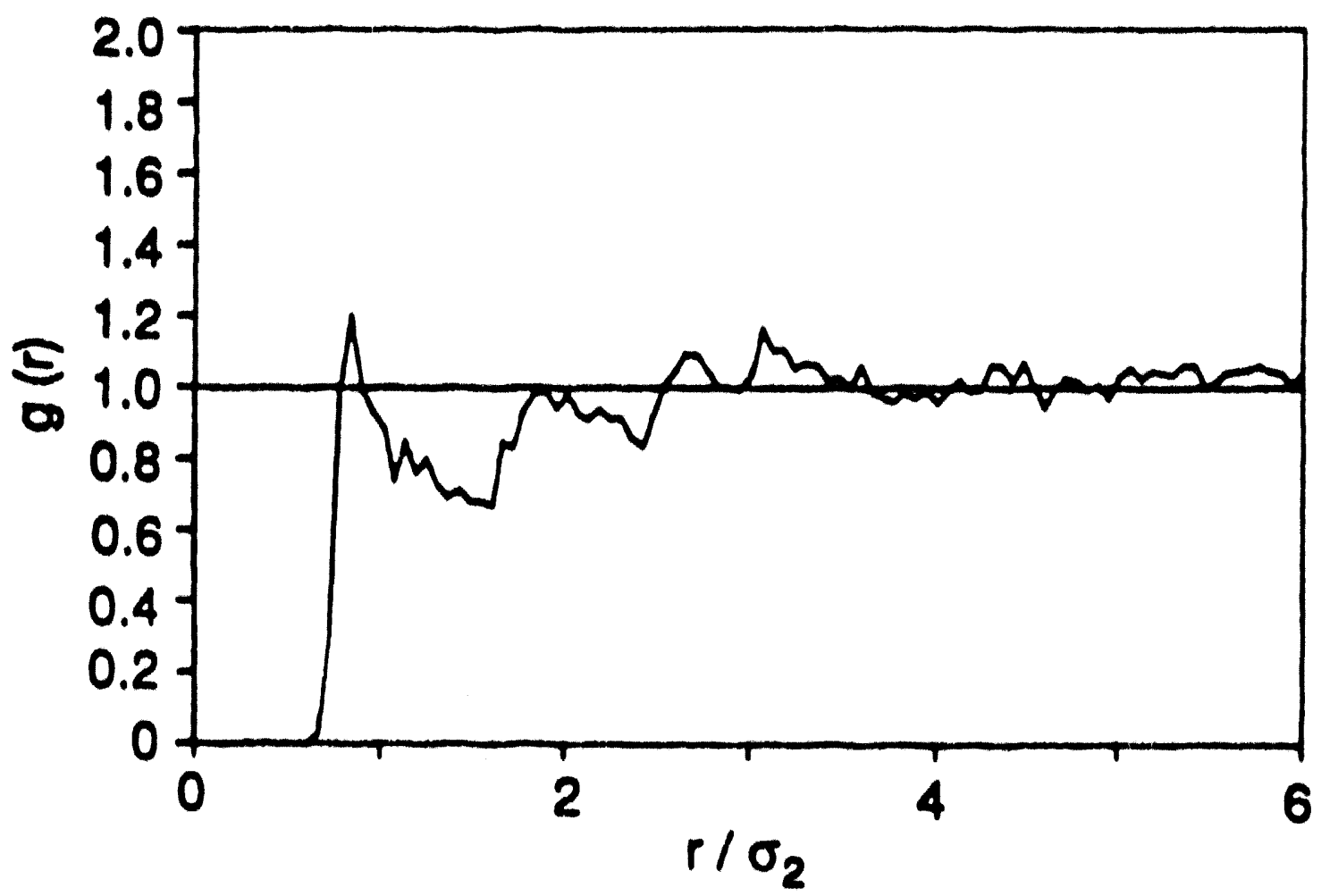

(b)

Figure 5.7 Solute-solvent pair correlation functions for the repulsive mixture at $T^{*}=1.4, \rho^{*}=0.35$. Each curve is an average over 5000 steps, spanning 22.1 ps. Noise is indicative of weakened solute-solvent correlations and enhanced density fluctuations near the critical point of the solvent. Potential parameters as per Table 4. Molecular dynamics simulation. (From Petsche, I. B. and Debenedetti, P. G., J. Chem. Phys., 91, 7075, 1989. With per- 


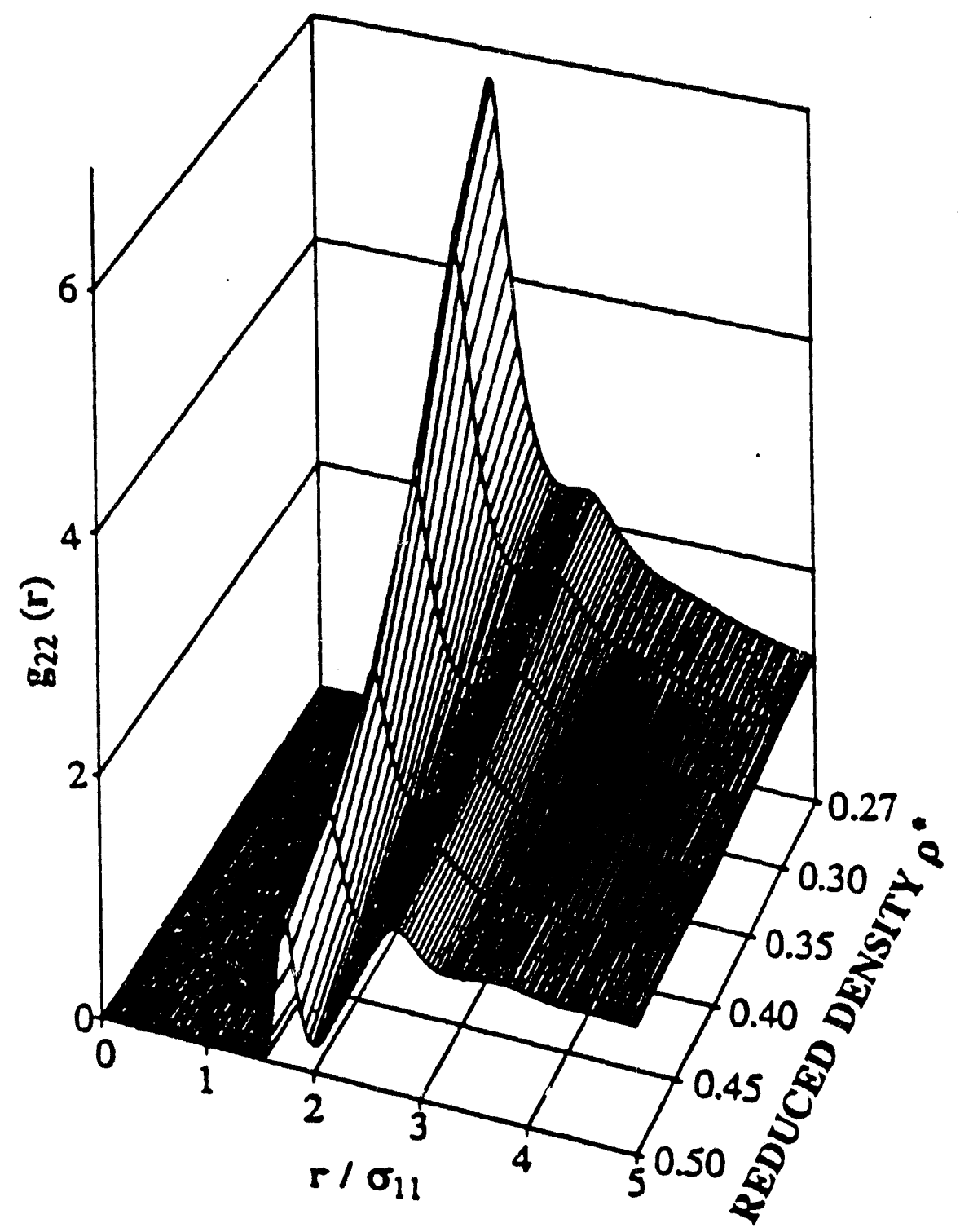

Figure 6.1 Solute-solute pair correlation functions $g_{22}(r)$ at $T^{*}=$ $1.415=1.096 T_{c}{ }^{*}$ and different densities. Pyrene-carbon dioxide mirture. PY closure. Solute mole fraction $=10^{-9}$. 


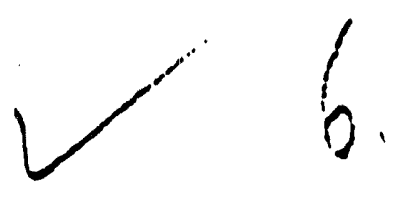

Solute Solute
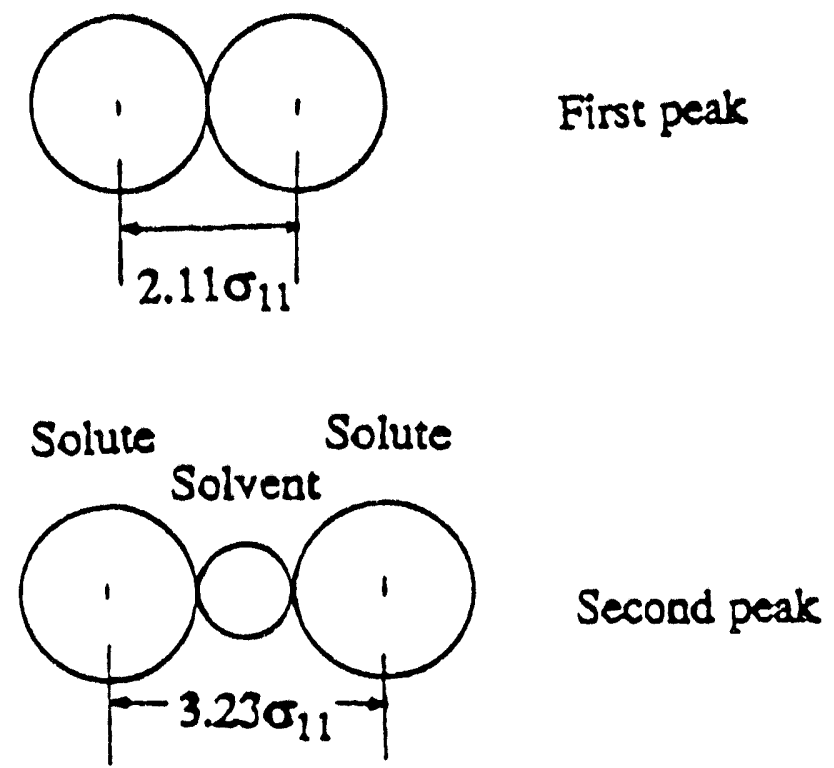

Solute Solute Solute

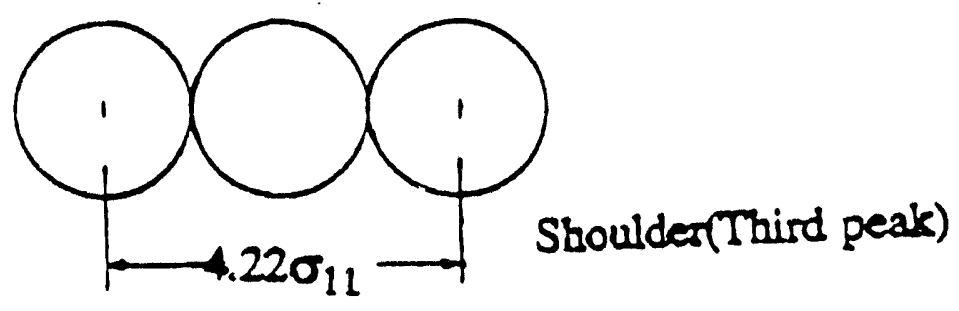

Figure 6.2 . Radial packing geometry of $g_{22}(r)$ peaks.

Table III. Lennard-Jones Parameters

\begin{tabular}{lcc}
\hline & $\epsilon / k, \mathrm{~K}$ & $\sigma, \AA$ \\
\hline monomer-monomer, mm & 225.3 & 3.794 \\
dimer site-dimer site, ss & 225.3 & 5.691 \\
monomer-dimer site, ms & 225.3 & 4.743
\end{tabular}




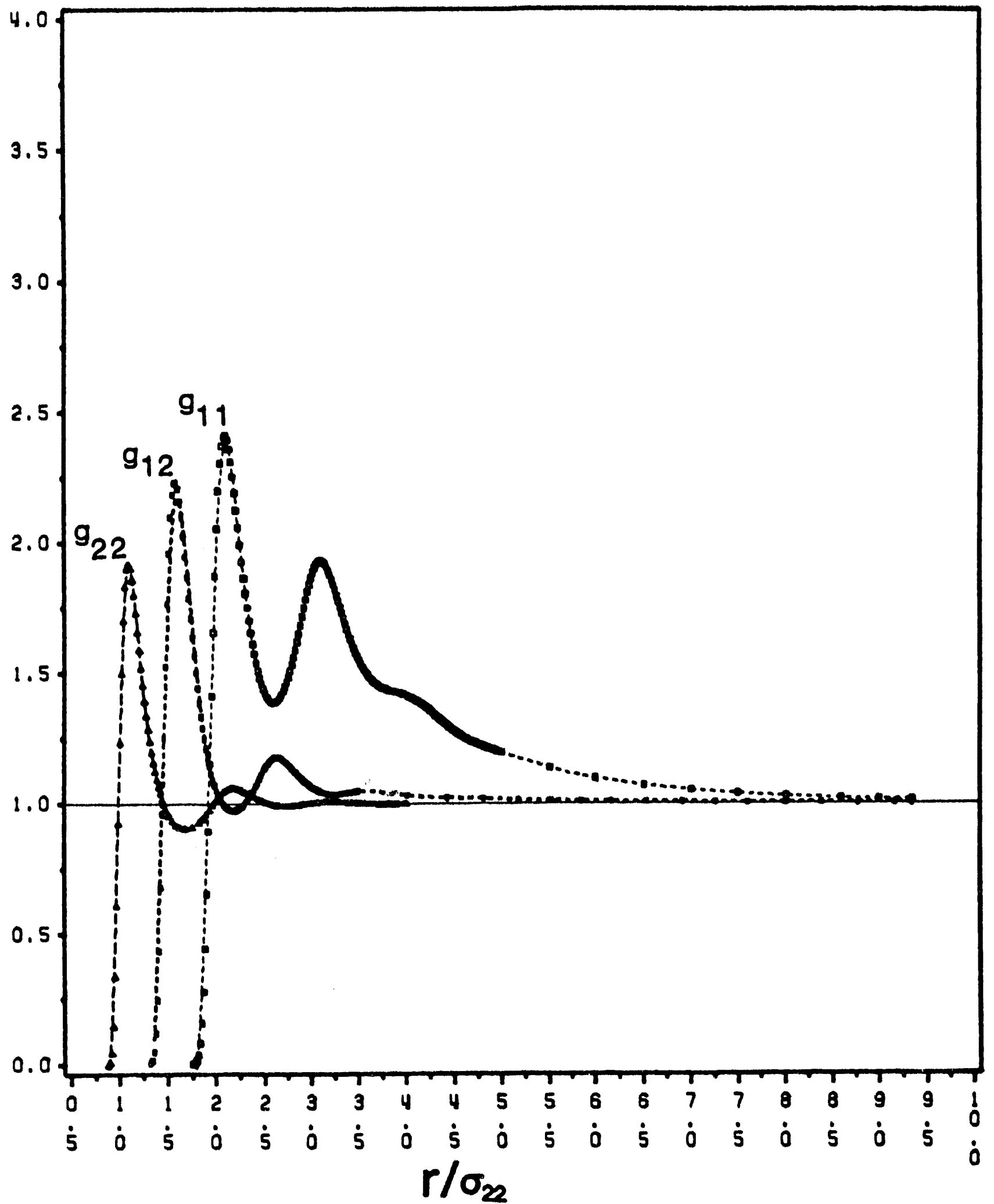

Figure 6.3 Pair correlation functions for solute-solute (squares), solute-solvent (stars), and solvent-solvent (triangles) interactions at $\mathrm{T}^{*}=1.50, \rho^{*}=0.4248$. Potential parameters as per Table 3 (pyrene-carbon dioxide). RHNC closure. Solute mole fraction $=3 \times 10^{-7}$. (From Wu, R.-S., Lee, L. L., and Cochran, H. D., Ind Ene. Chem. Res., 29, 977, 1990. With permission.) 


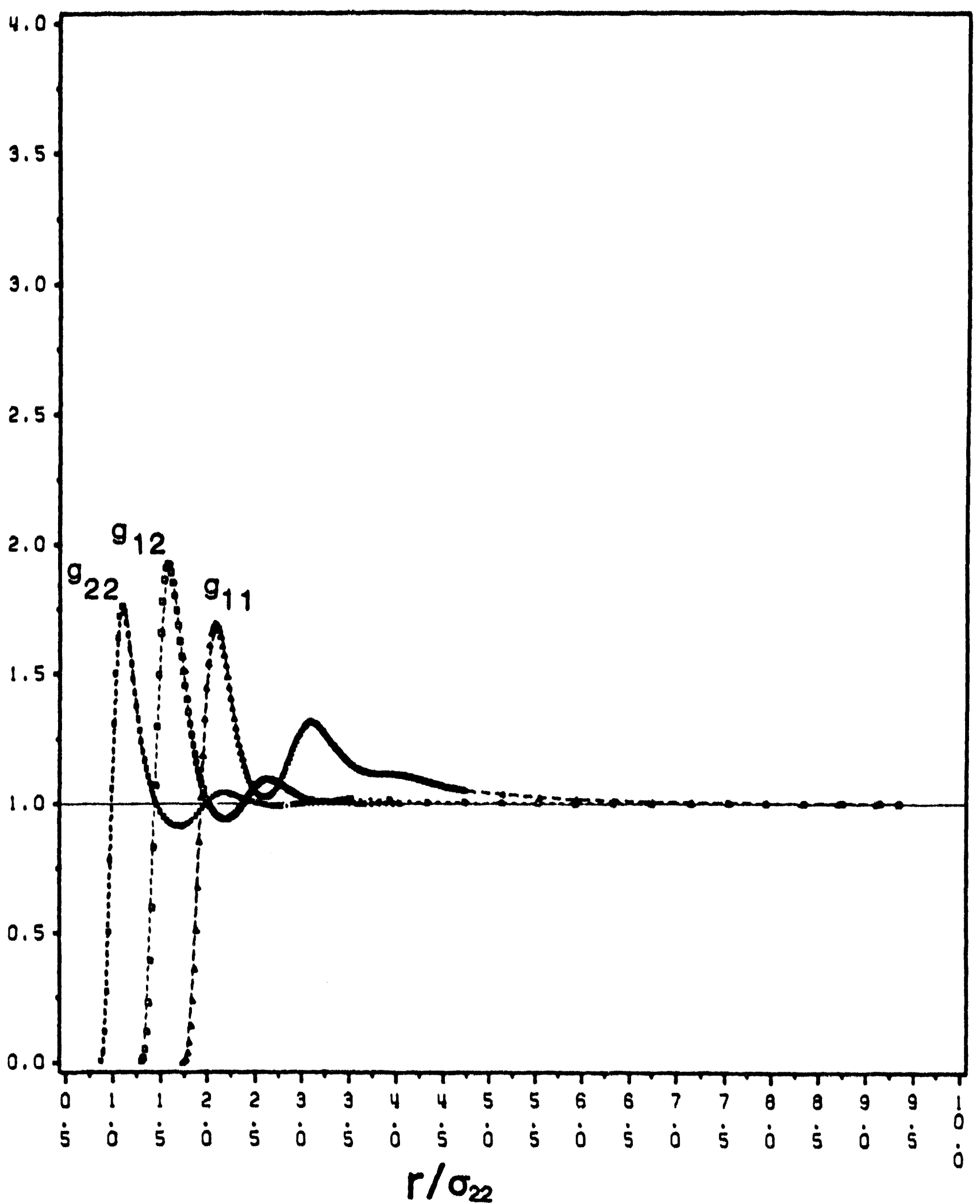

igure 6.3(b) Pair correlation functions for solute-solute (squares), solute-solvent (stars), and solvent-solvent (triangles) interactions at $\mathrm{T}^{*}=2.00, \rho^{*}=0.4248$. Potential parameters as per Table 3 (pyrene-carbon dioxide). RHNC closure. Solute mole fraction $=3 \times 10^{-1}$. (From Wu, R.S., Lee, L. L., and Cochran, H. D., Ind. 


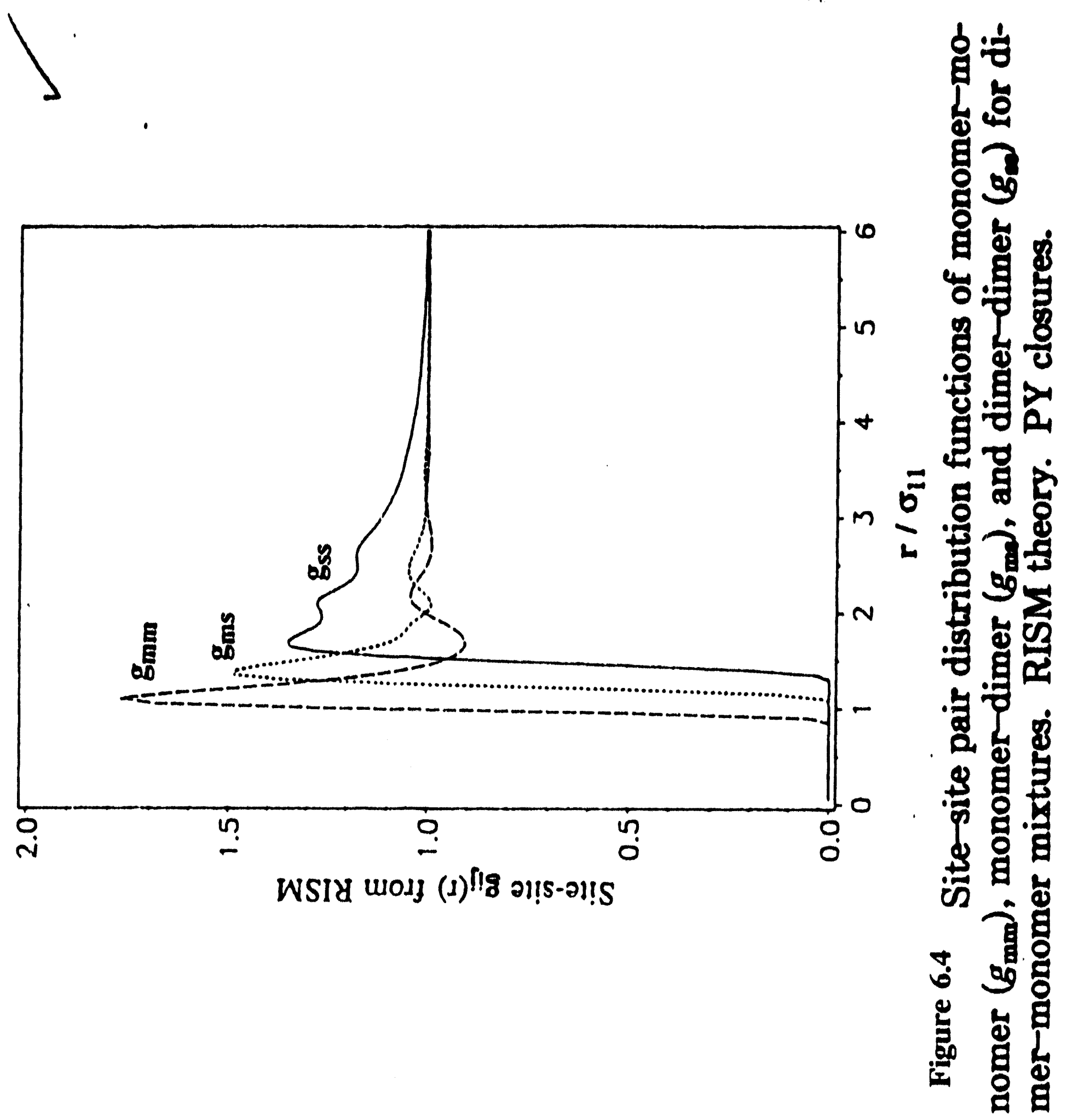



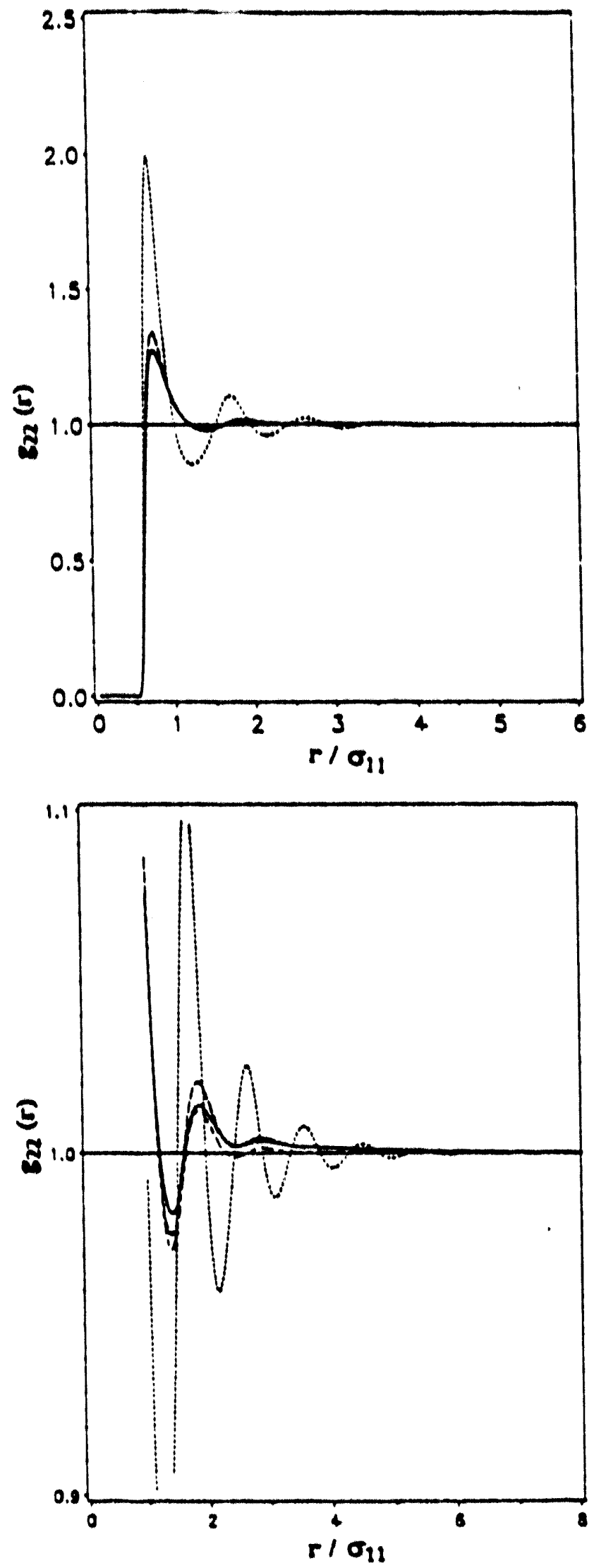

Figure 6.5 (a, top) Solute-solute pair correlation functions $g_{2}(r)$ at $T^{*}=2.0, \rho^{*}=0.35(--) ; T^{*}=1.4, \rho^{*}=0.35(--) ;$ and $T^{*}=1.4$, $\rho^{*}=0.8(\ldots)$ for dilute $\mathrm{Ne}_{\mathrm{e}}$ in Xe (repulsive mixture). PY closure. Solute mole fraction $=10^{-0}$. (b, bottom) Solute-solute pair correlation functions $g_{22}(r)$ at $T^{*}=2.0, \rho^{*}=0.35(---), T *=1.4, \rho^{*}=0.35$ $(--) ; T^{*}=1.34, \rho^{*}=0.27(-) ;$ and $T^{*}=1.4, \rho^{*}=0.8(\ldots)$ for dilute Ne in Xe (repulsive mirture). PY closure. Solute mole fraction $=$ $10 \rightarrow$. 


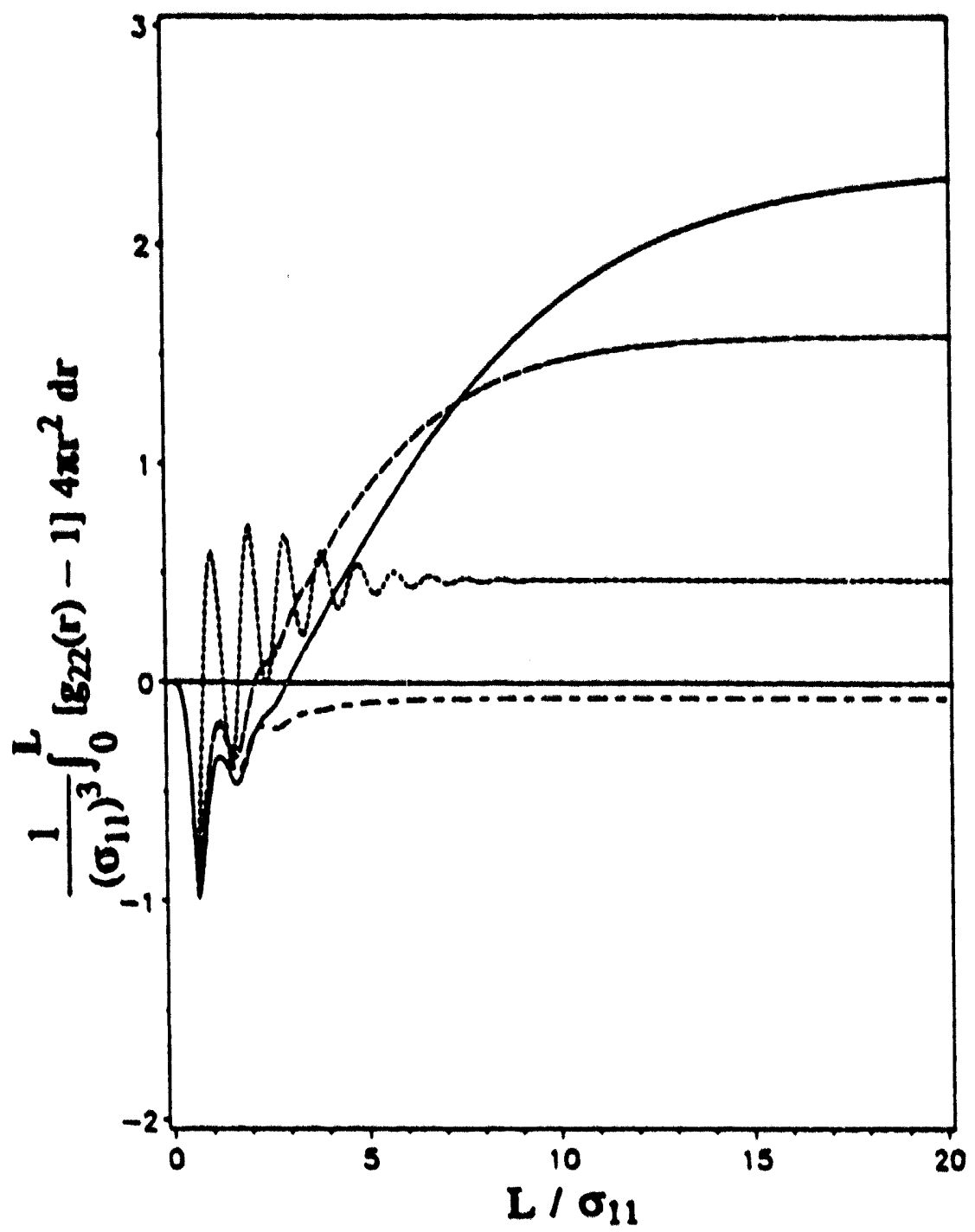

Figure 6.6 Number of excess solute molecules within a sphere of radius $L$ around a solute, at $T^{*}=2.0, \rho^{*}=0.35(---) ; T^{*}=1.4, \rho^{*}$ $=0.35(--) ; T^{*}=1.34, \rho^{*}=0.27(-) ;$ and $T^{*}=1.4, \rho^{*}=0.8(-\cdot)$ for dilute $\mathrm{Ne}$ in $\mathrm{Xe}$ (repulsive mixture). PY closure. Solute mole fraction $=10^{-\rightarrow}$. 


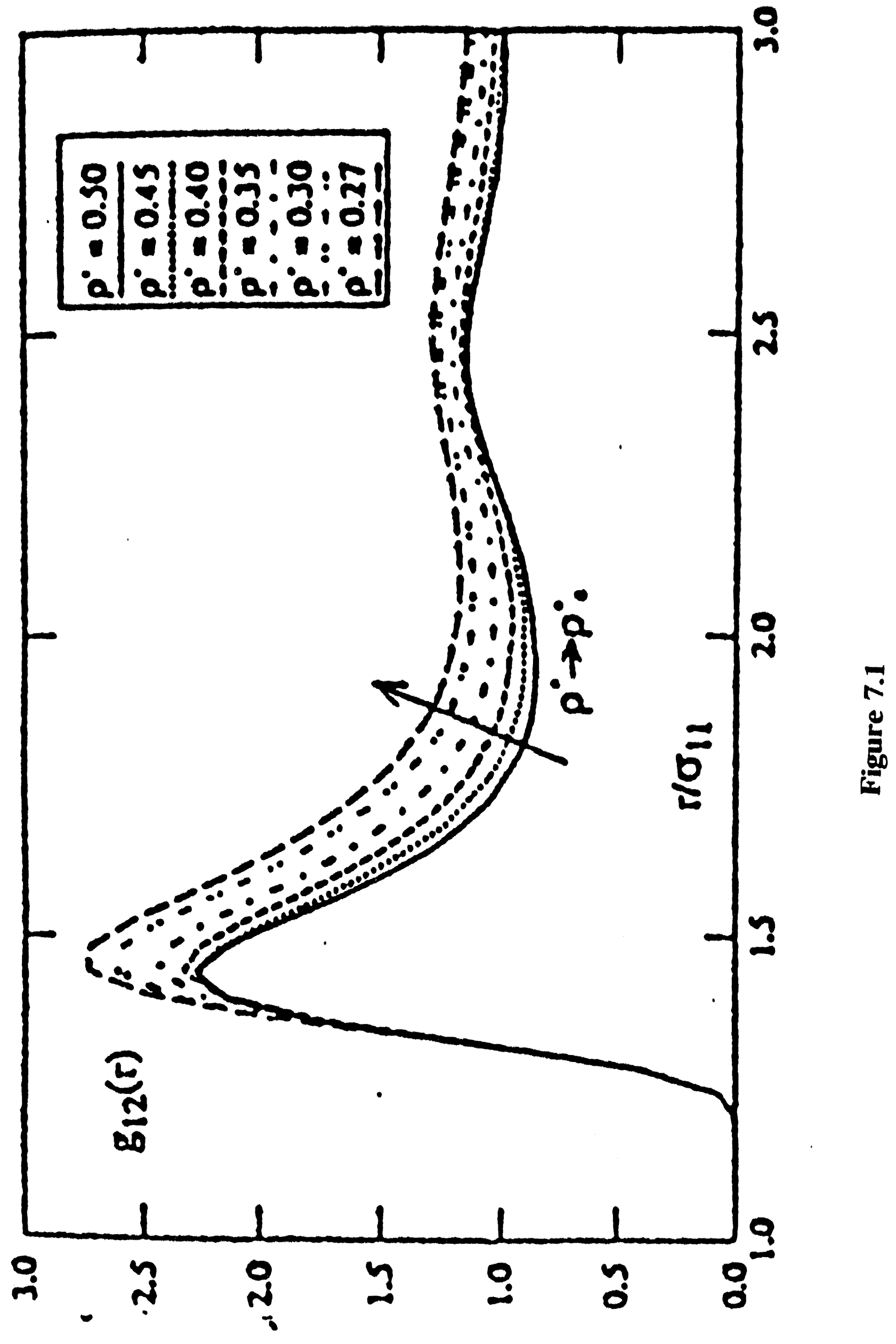




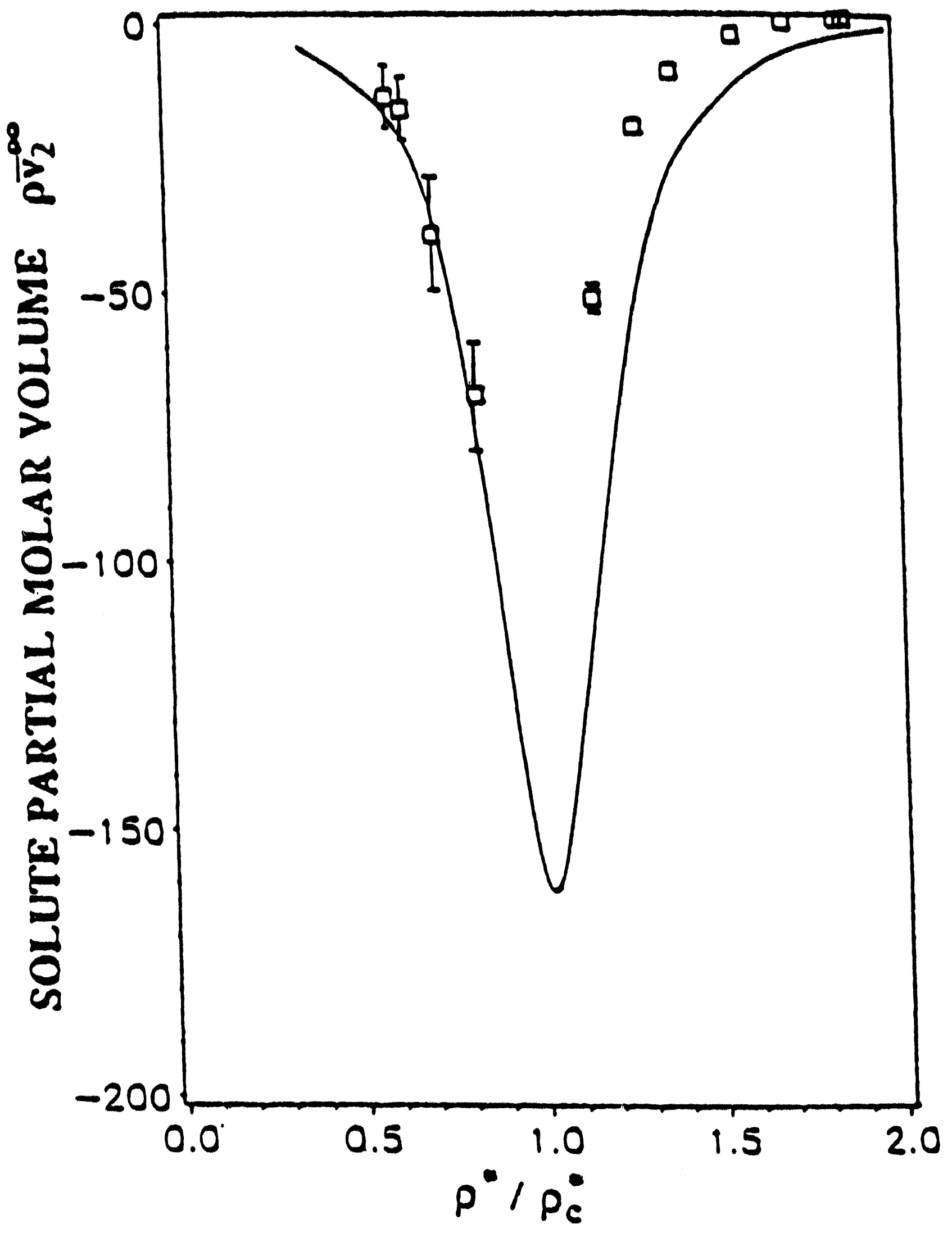

Figure $\mathbf{7 . 2}$ 


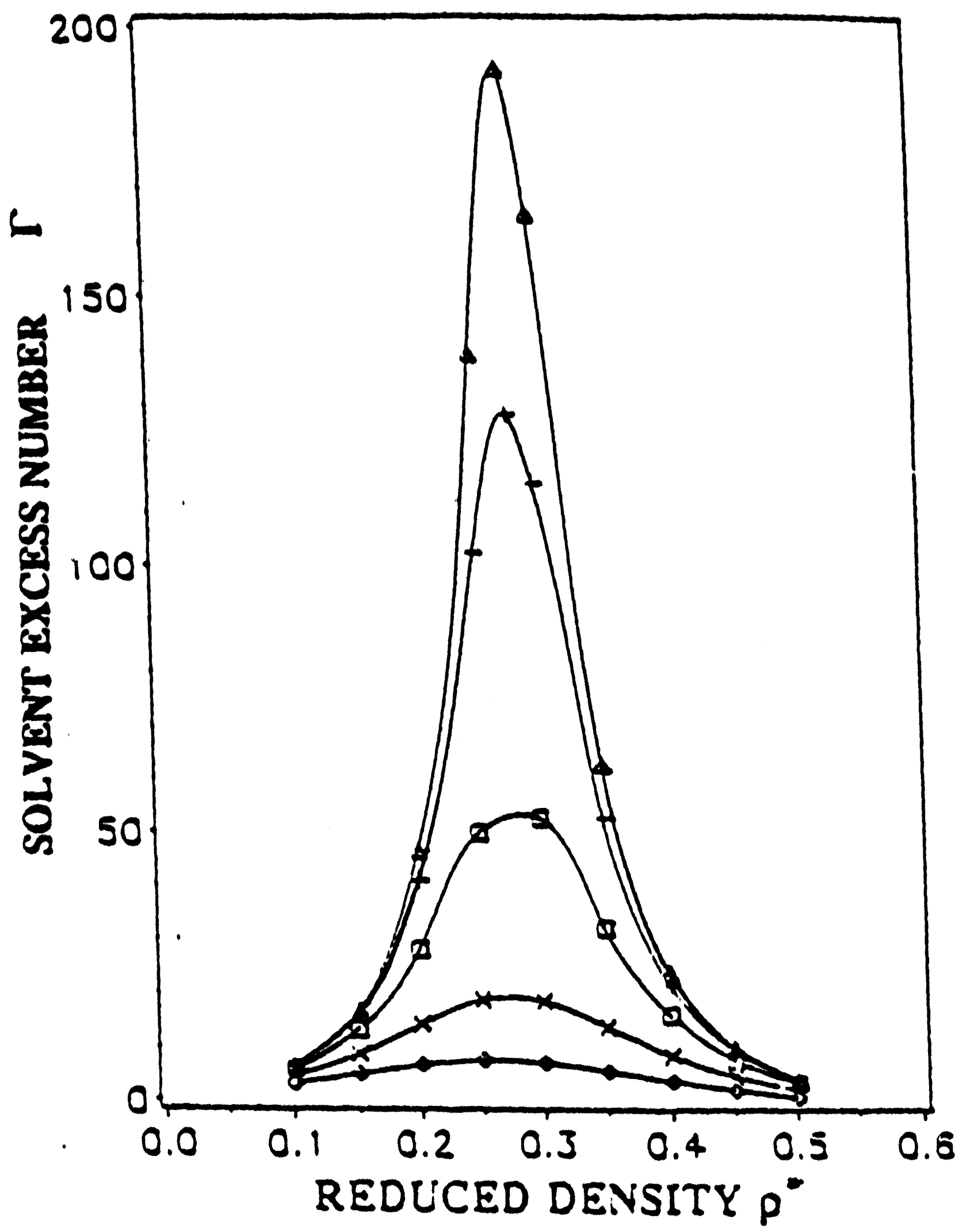

Figure 7.3 (a) 


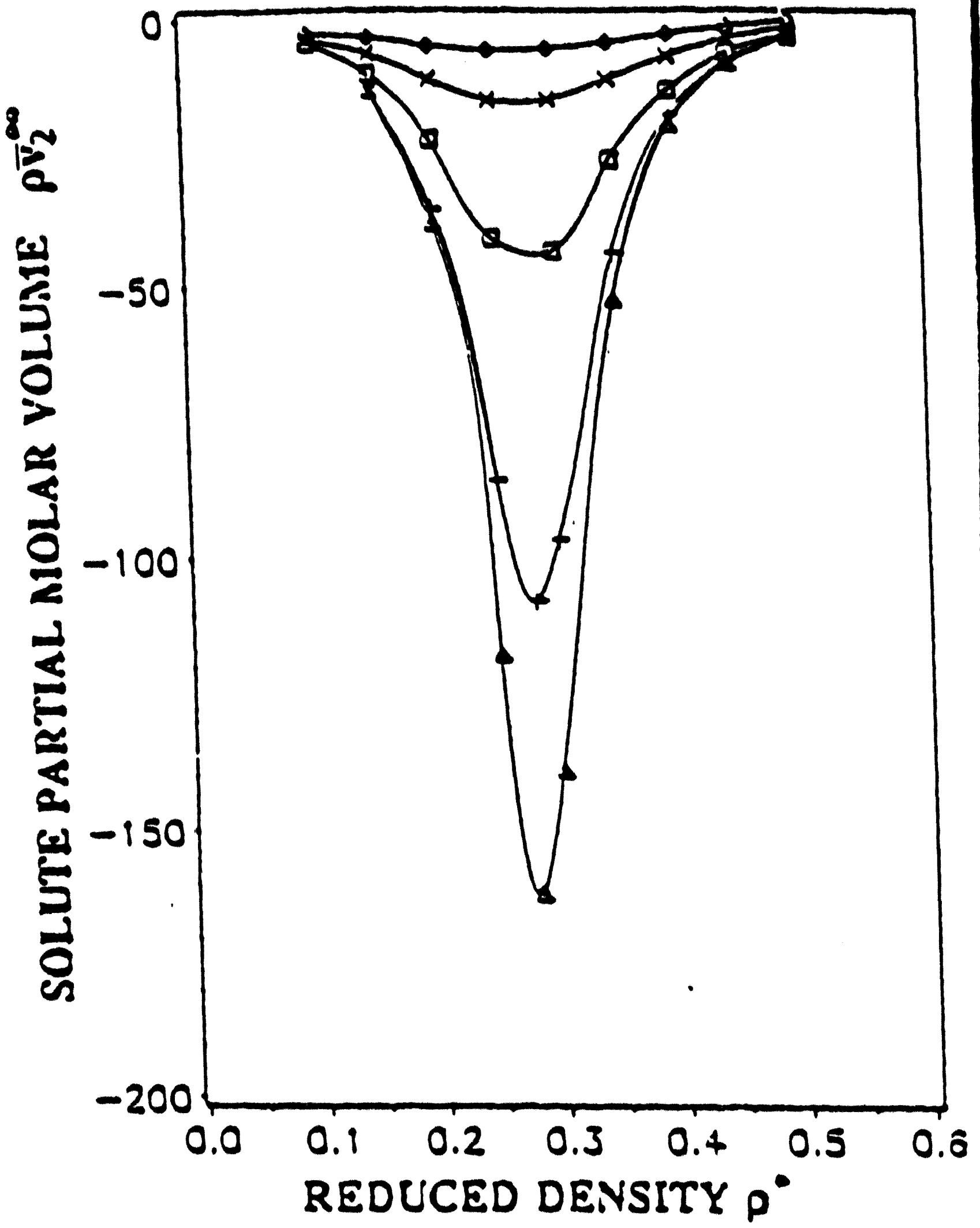

Figure 7.3 (b) 


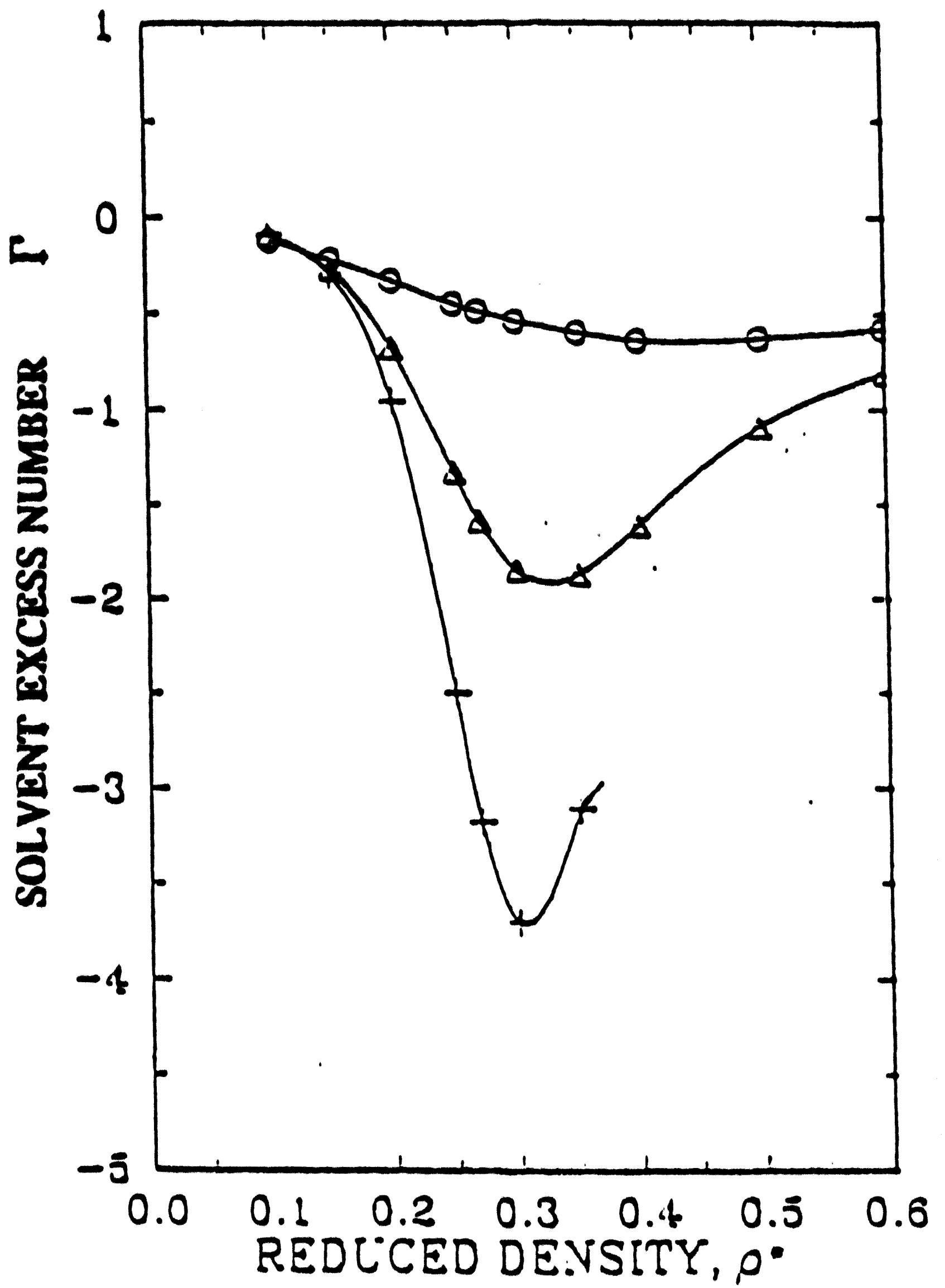

Figure 7.4 (a) 


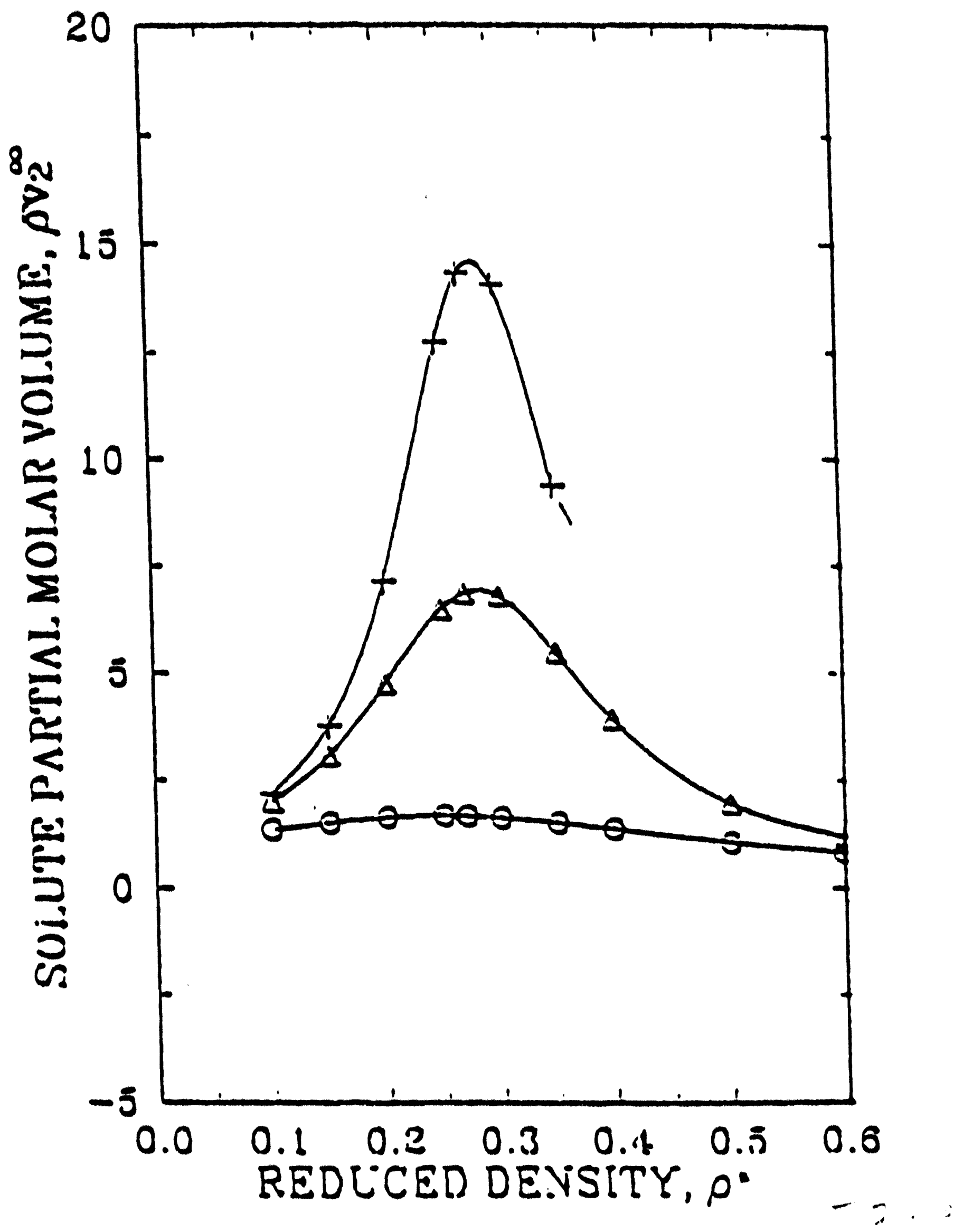

Figure 7.4 (b) 


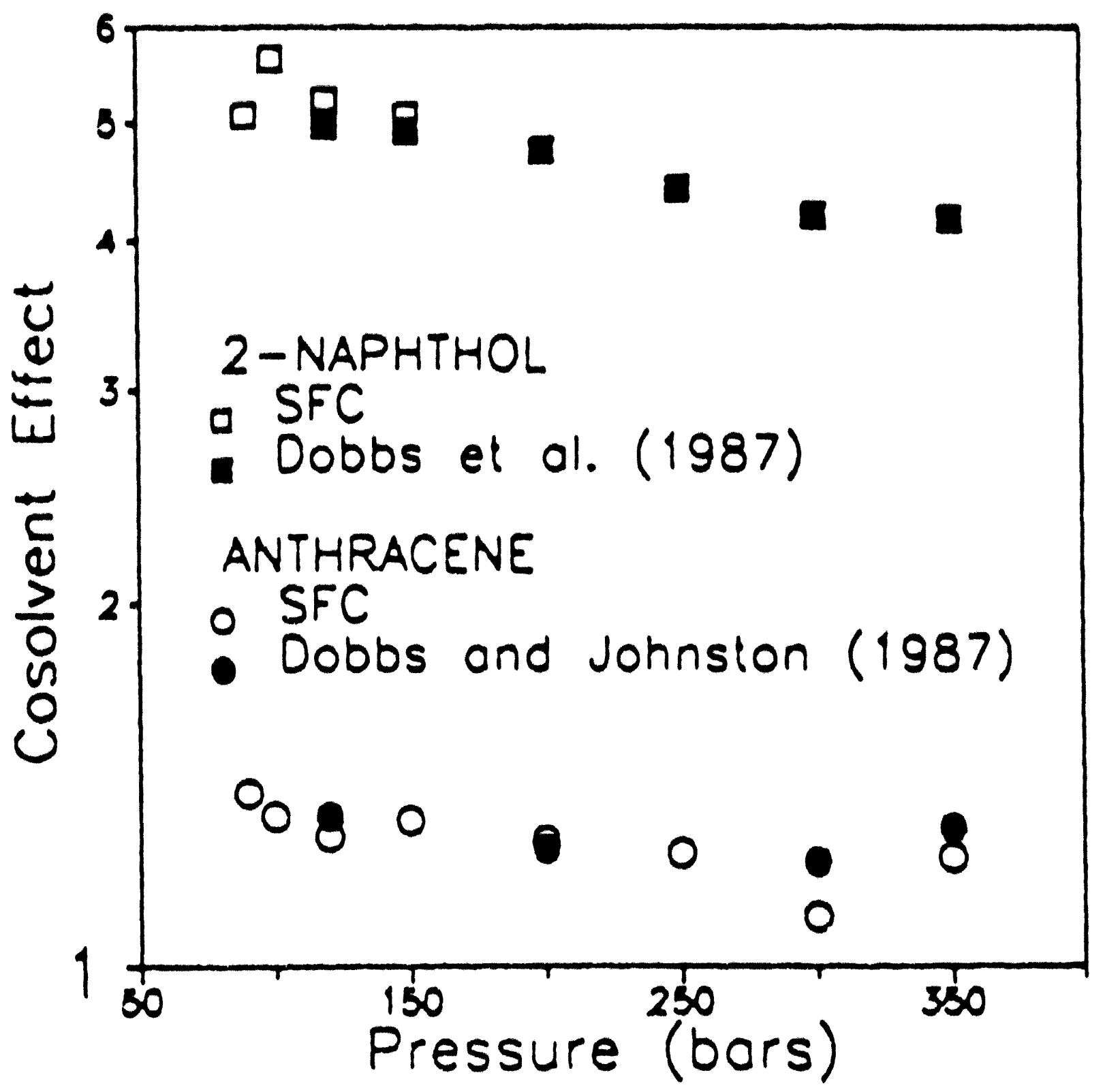

Figure 8.1 Cosolvent effect for 2.naphthol and anthra. cene in $\mathrm{CO}$ : with $3.5 \mathrm{~mol}$ percent methanol at $35^{\circ} \mathrm{C}$.

Condrent effect is ratro of solutilits in $\mathrm{CO}$ : methand mivure :0 11s solurilie! in pure $C O$ : 


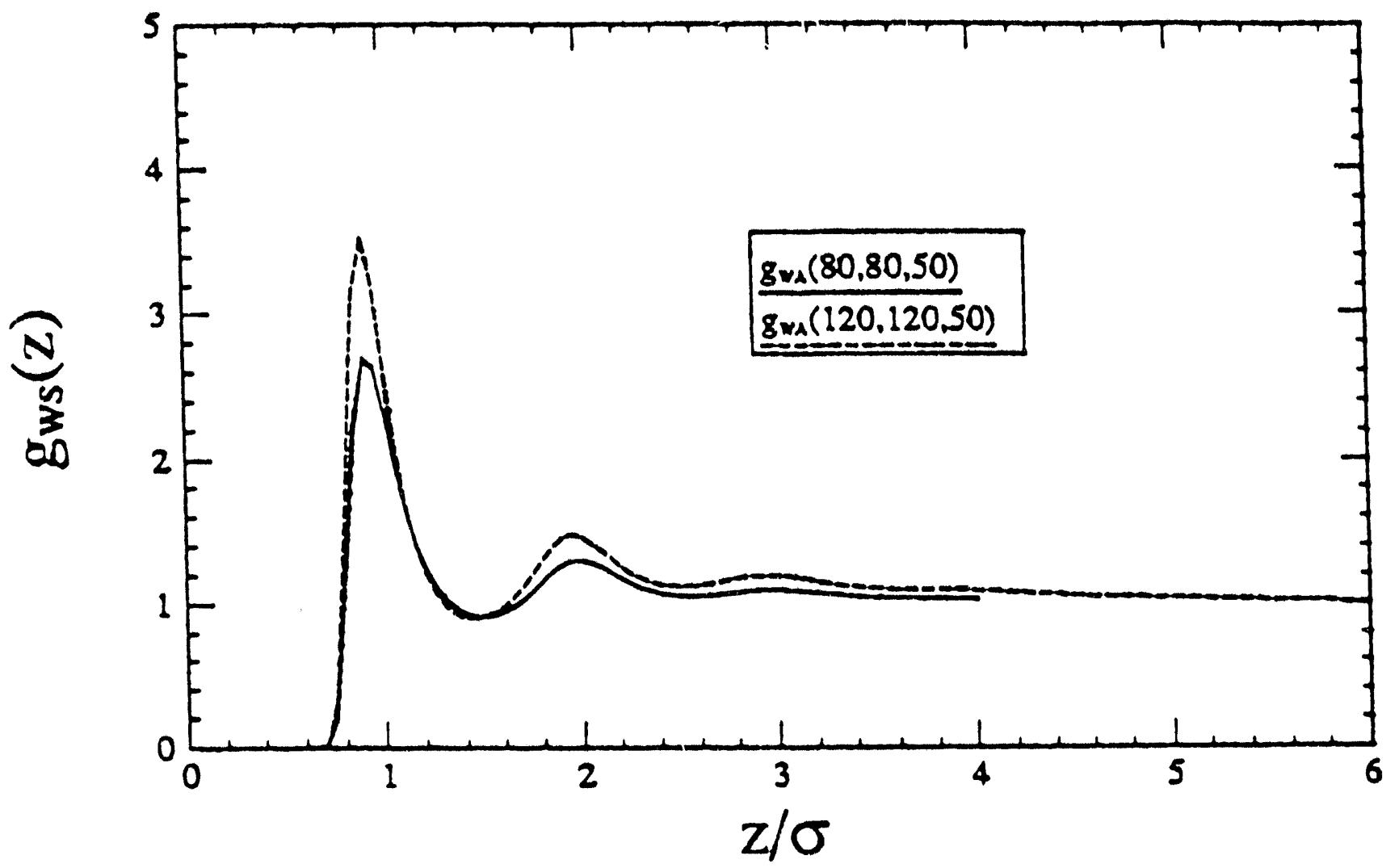

Figure 9.1 (a) Effect of range on wall-particle pair correlation function gws at $T^{*}=1.35$ and $p^{*}=0.35$. Wall-solvent correlation $g W_{A}$. 


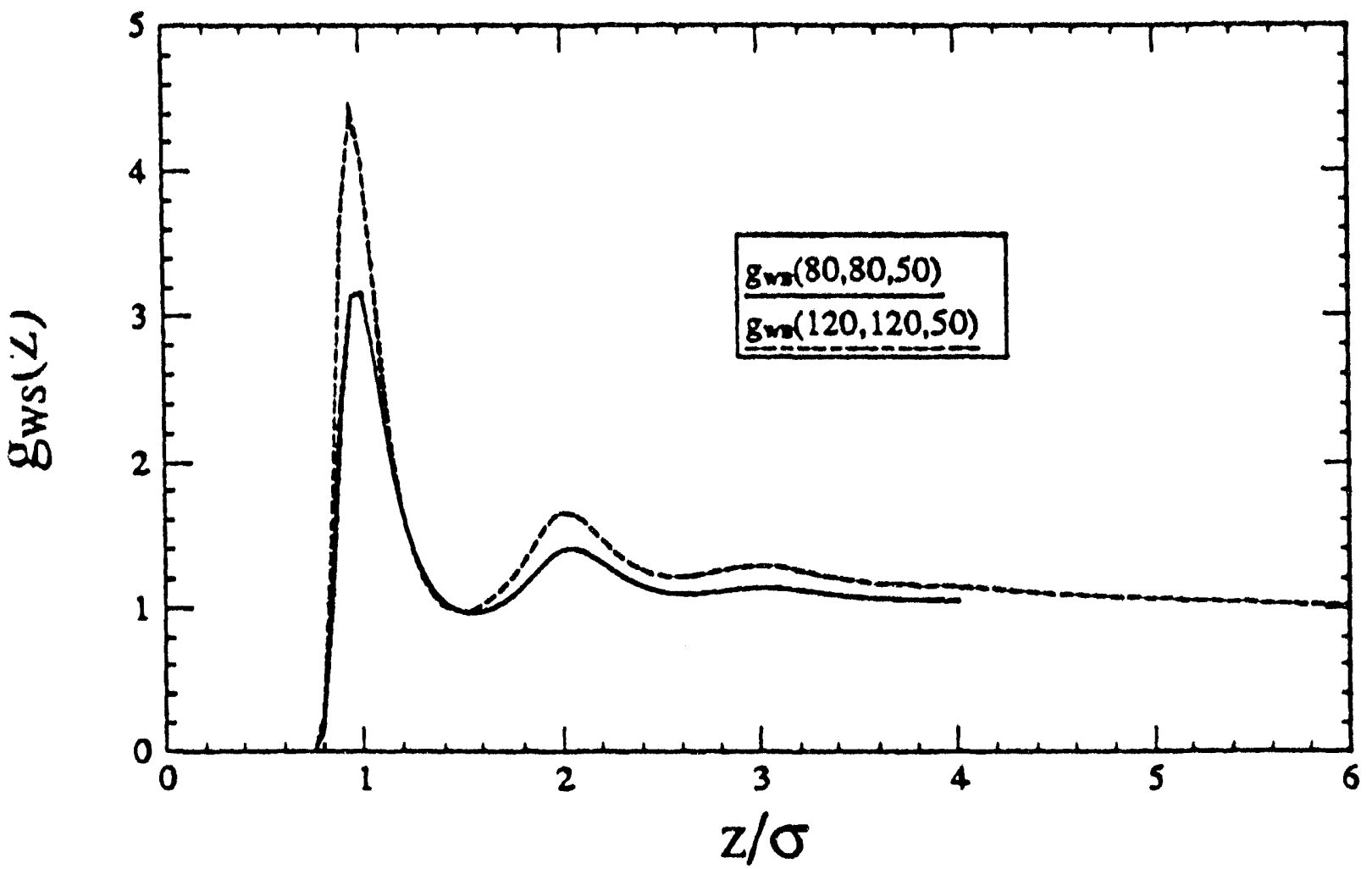

Figure 9.1 (b) Effect of range on wall-particle pair correlation function $g w s$ at $T^{*}=1.35$ and $\rho^{*}=0.35$. Wall-solute (attractive) correlation gwB. 


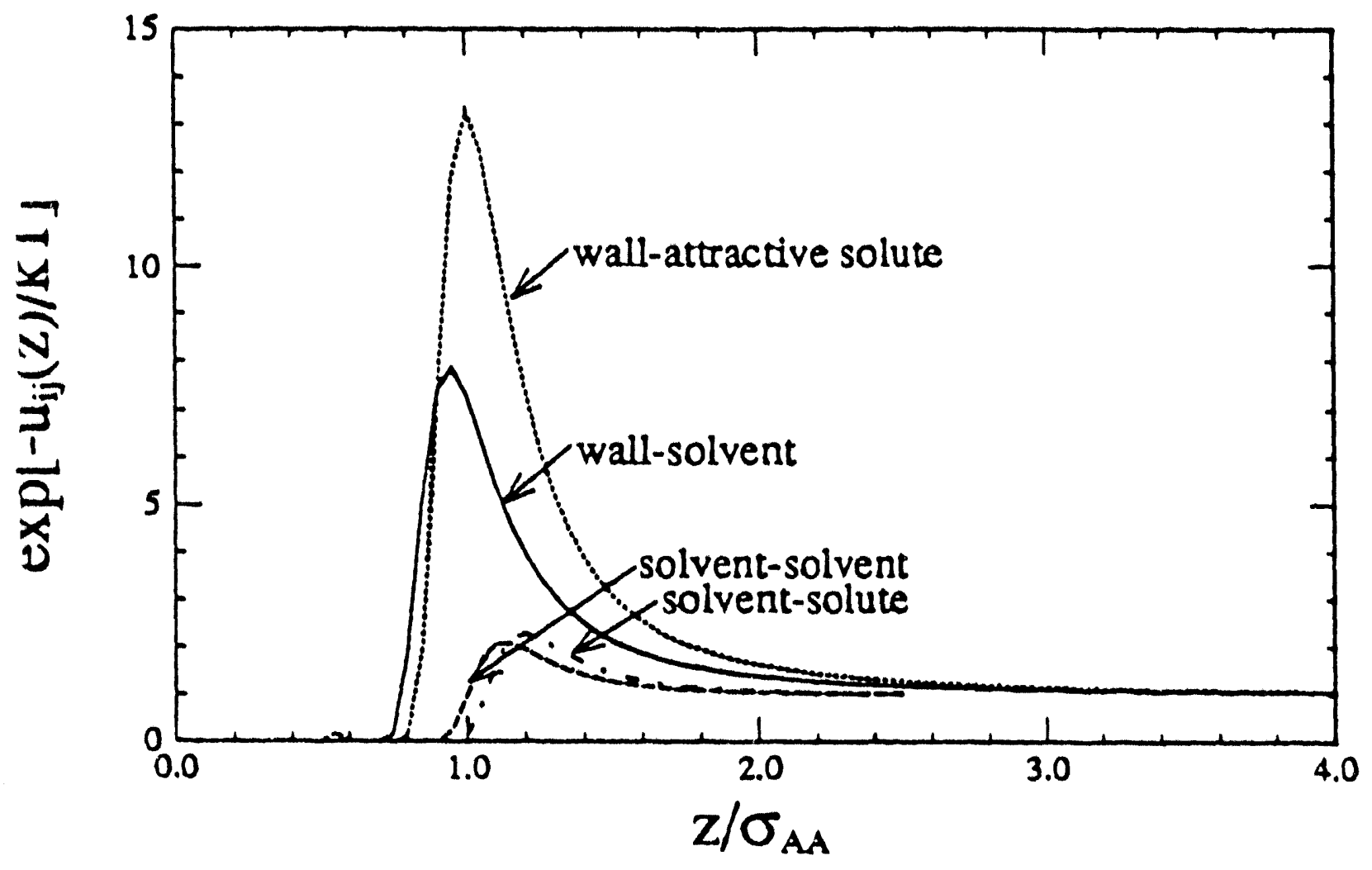

Figure 9.2 Boltzmann factor at $T^{*}=1.35$ for wall-particle and particleparticle interactions. 


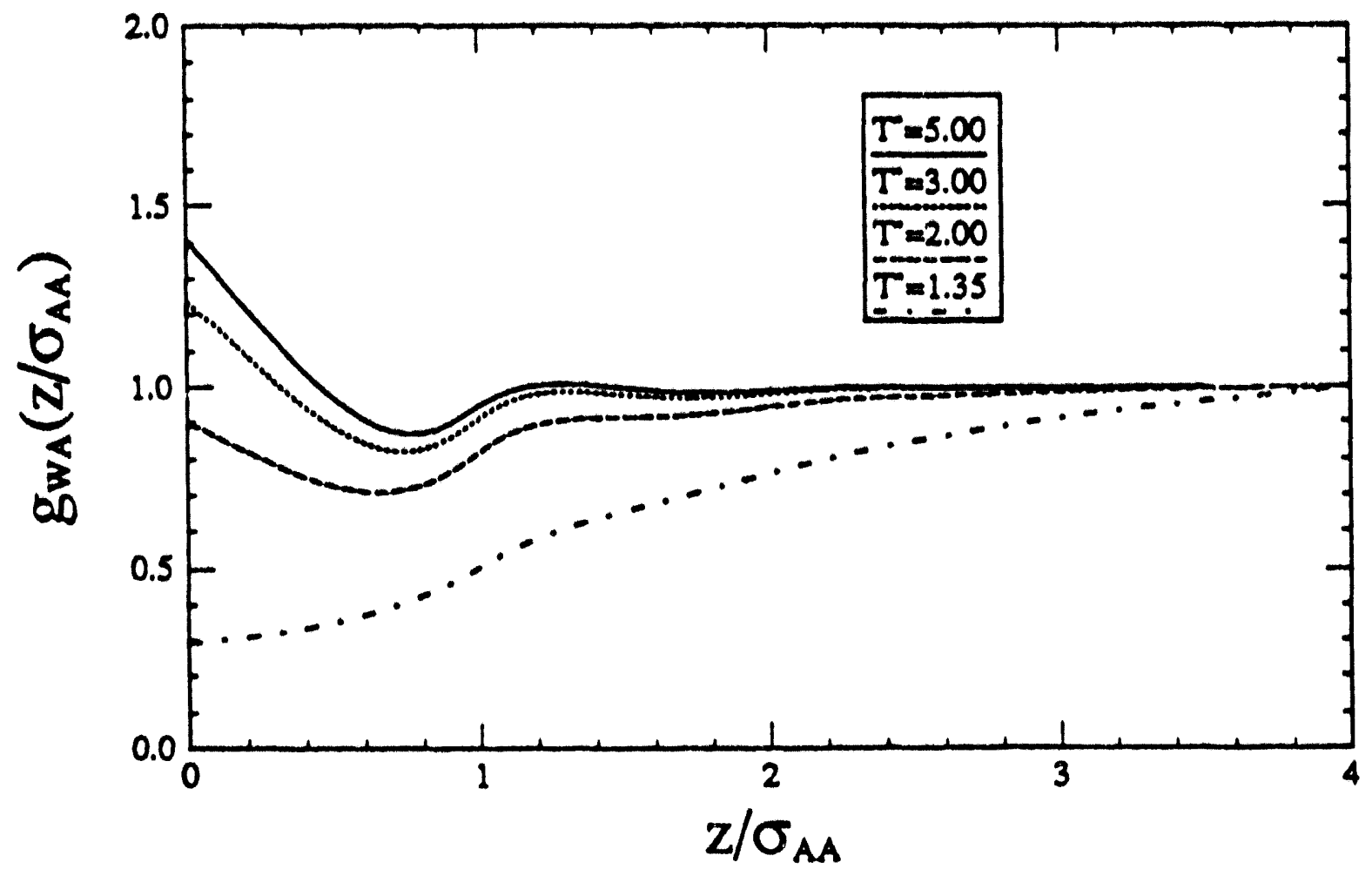

Figure 9.3 (a) Effect of temperature on density profiles near a hard wall. Wall-solvent profile $8 W_{A}$. 


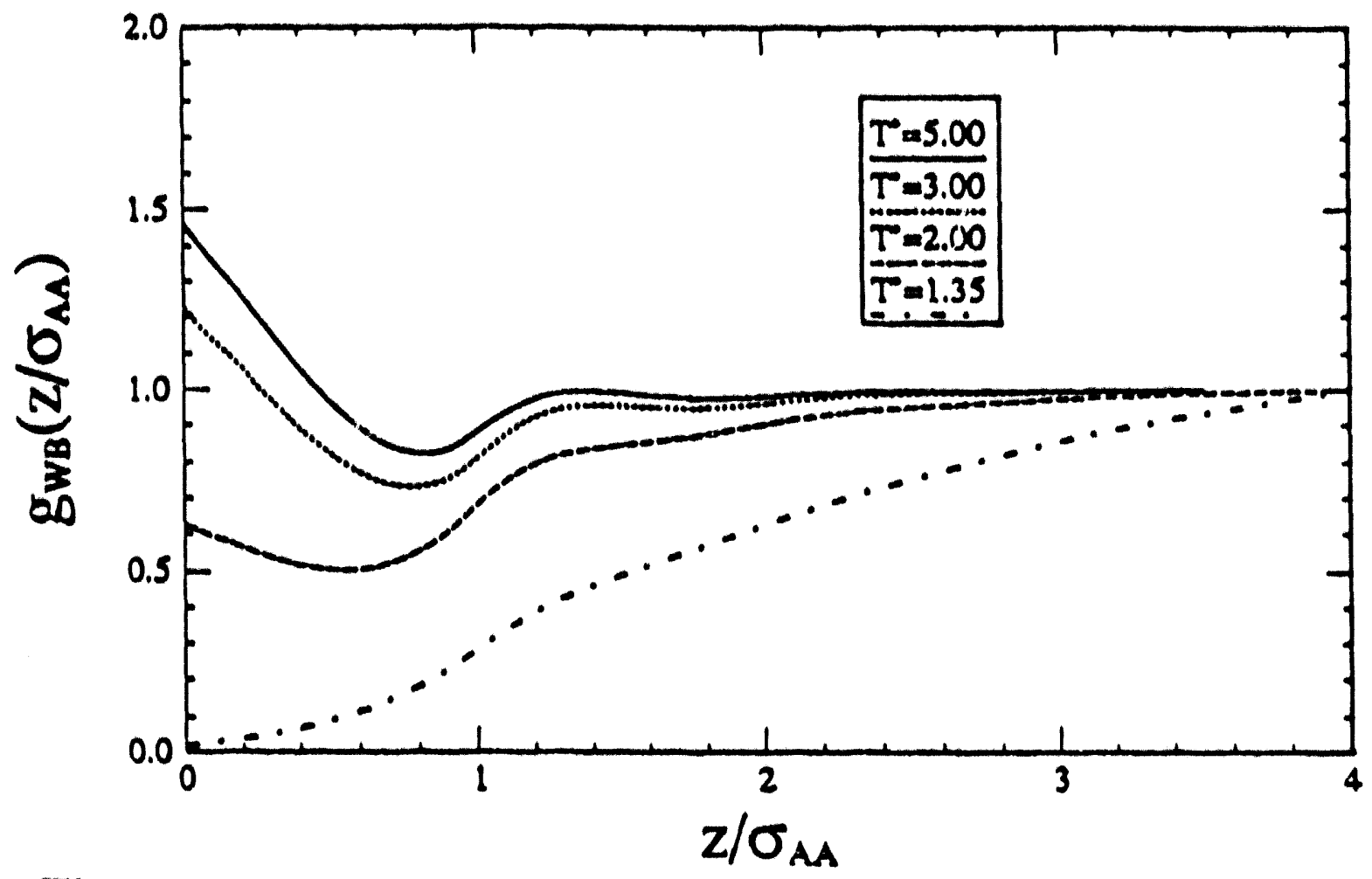

Figure 9.3 (b) Effect of temperature on density profiles near a hard wall. Wall-solute (repulsive) profile swB. 


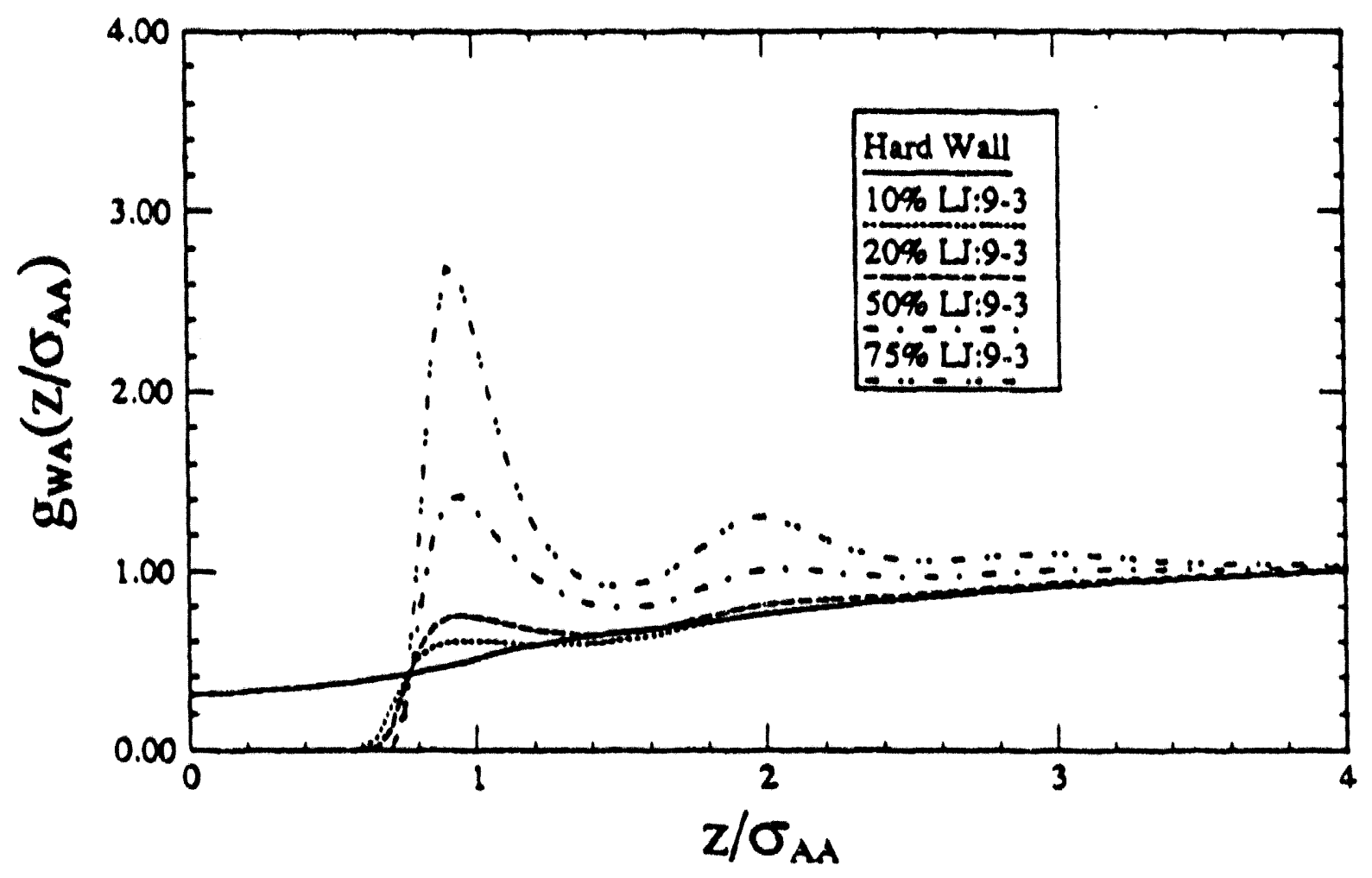

Figure 9.4 (a) Effect of wall strength on density profiles near $L J: 9-3$ wall. Wall-solvent profile 8 WA. 


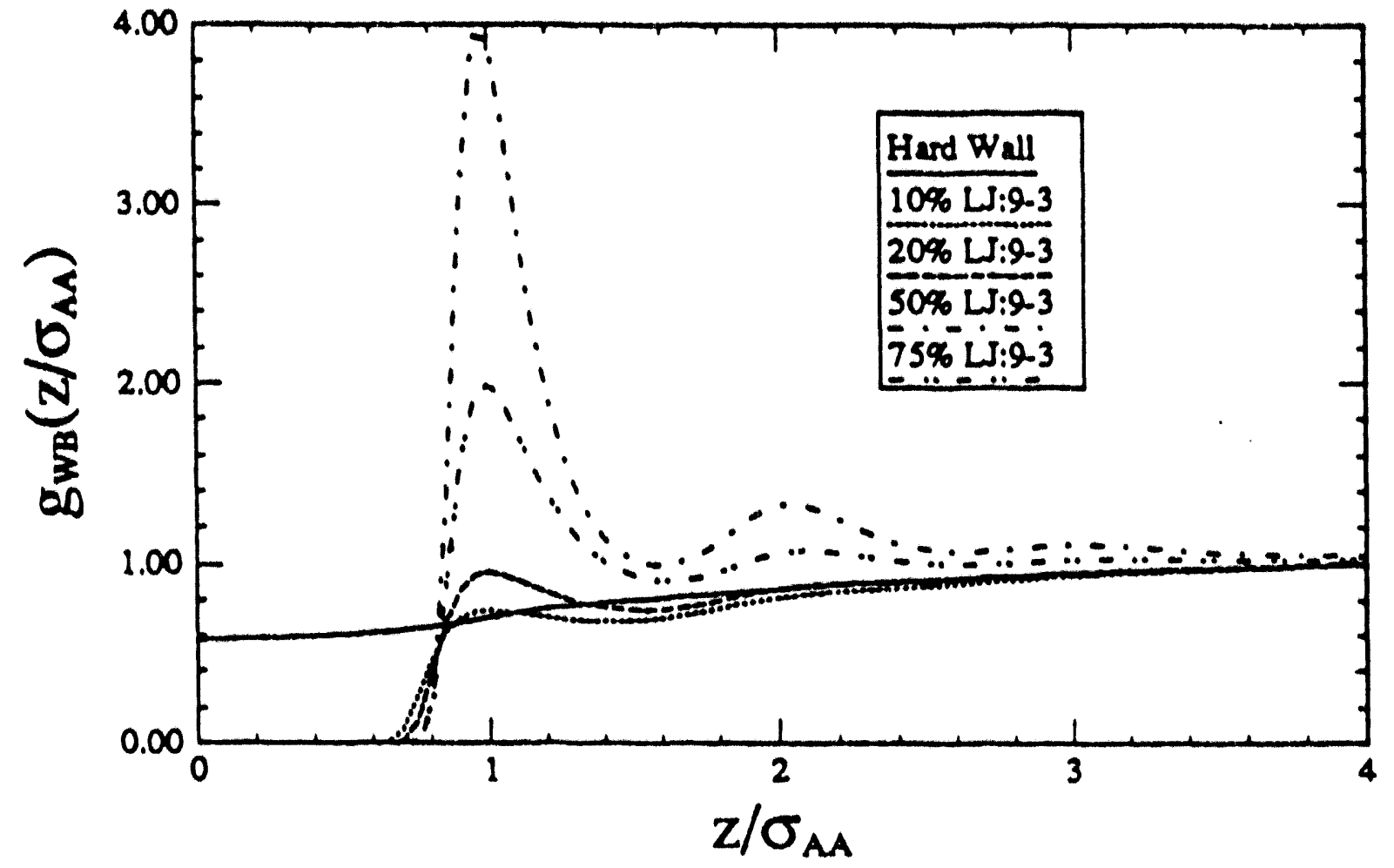

Figure 9.4 (b) Effect of wall strength on density profiles near $\mathrm{L}: 9-3$ wall. Wall-solute (repulsive) profile $g_{W B}$. 


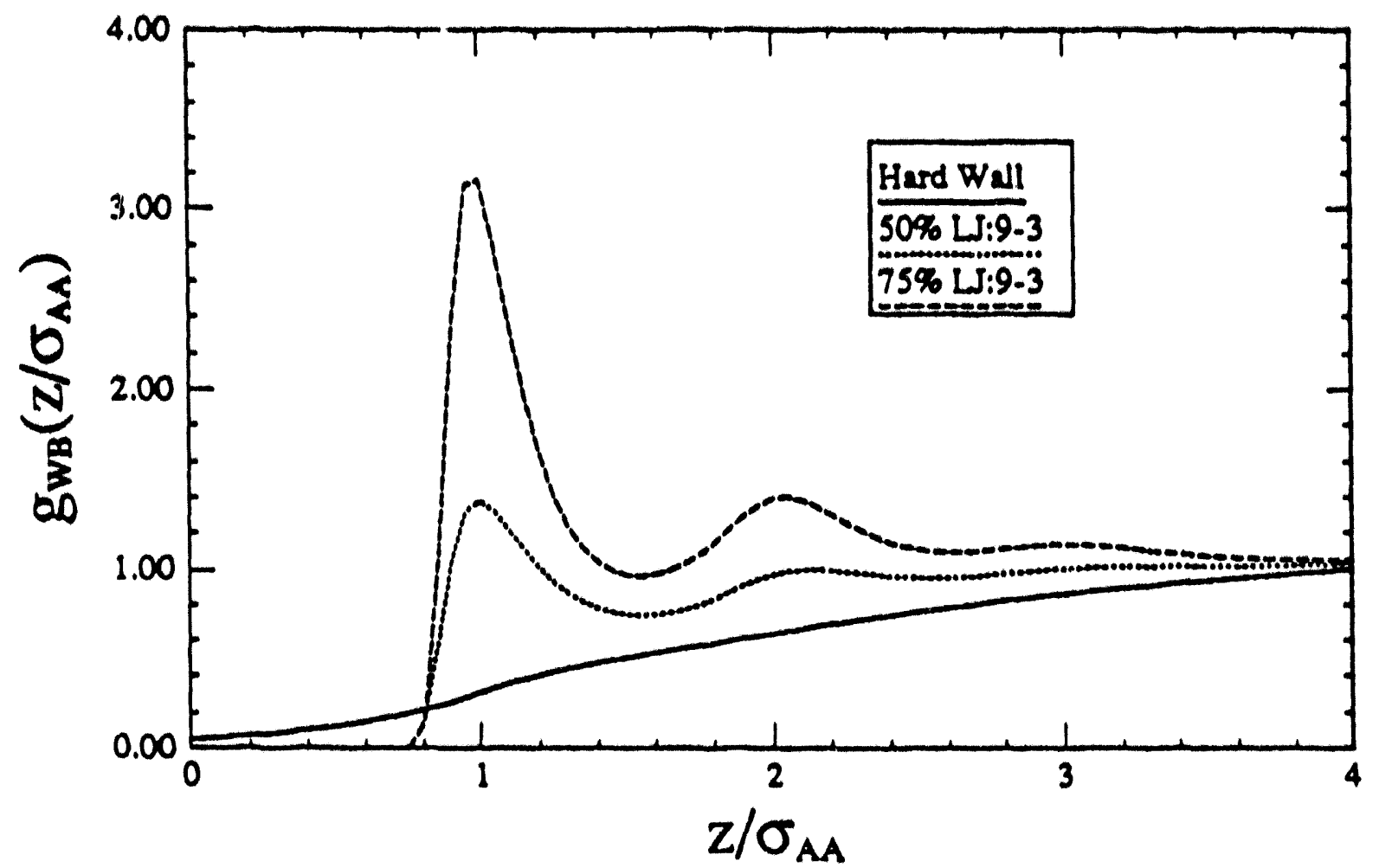

Figure $9.4{ }^{(c)}$ Effect of wall strength on density profiles near $\mathrm{WJ} 99-3$ wall. Wall-solute (attractive) profile swB. 


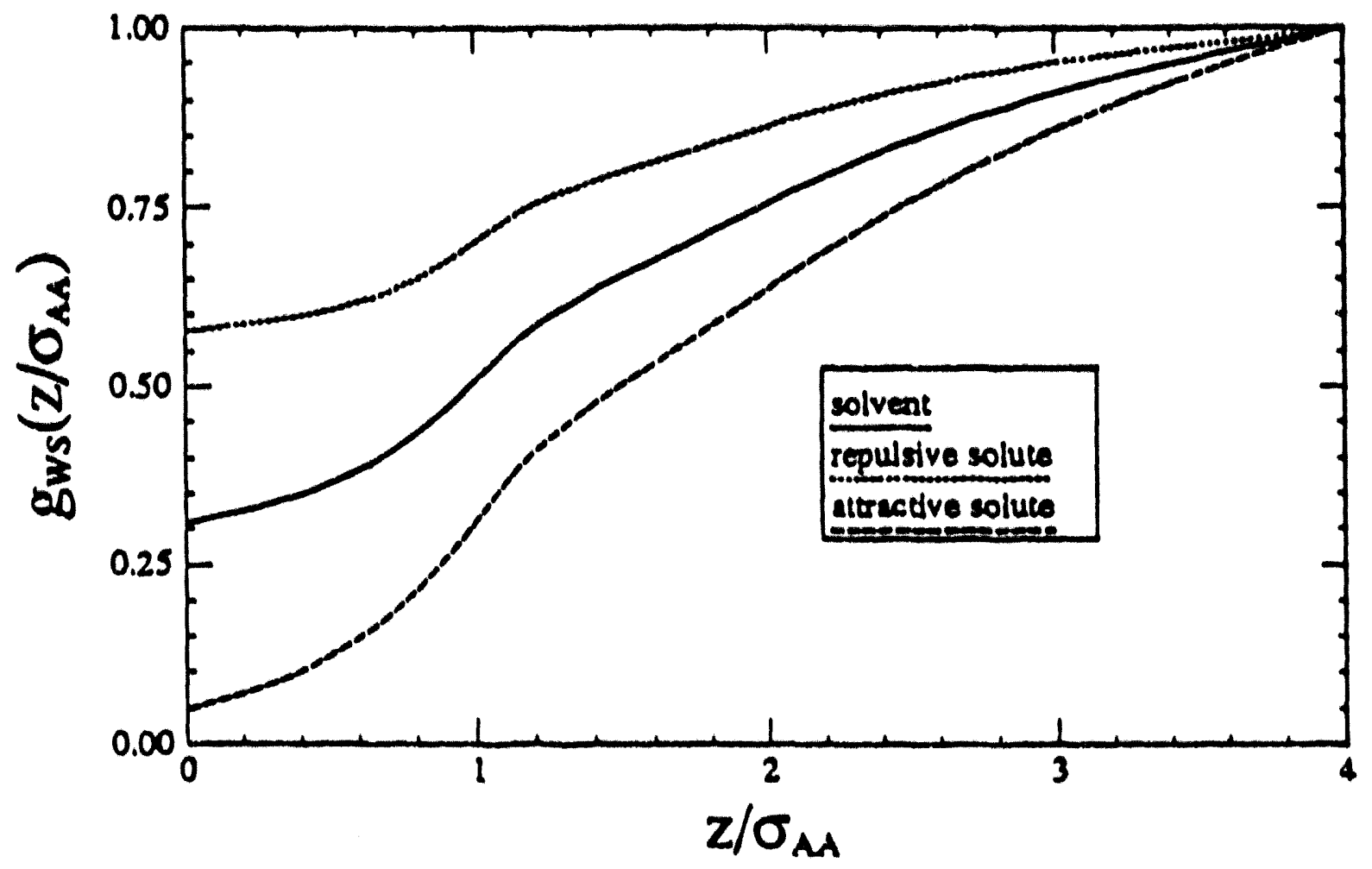

Figure 9.5 (a) Competitive adsorption between wall and repulsive or attractive solute. Wall-particle profiles gws are identified in the legend. Hard wall. 


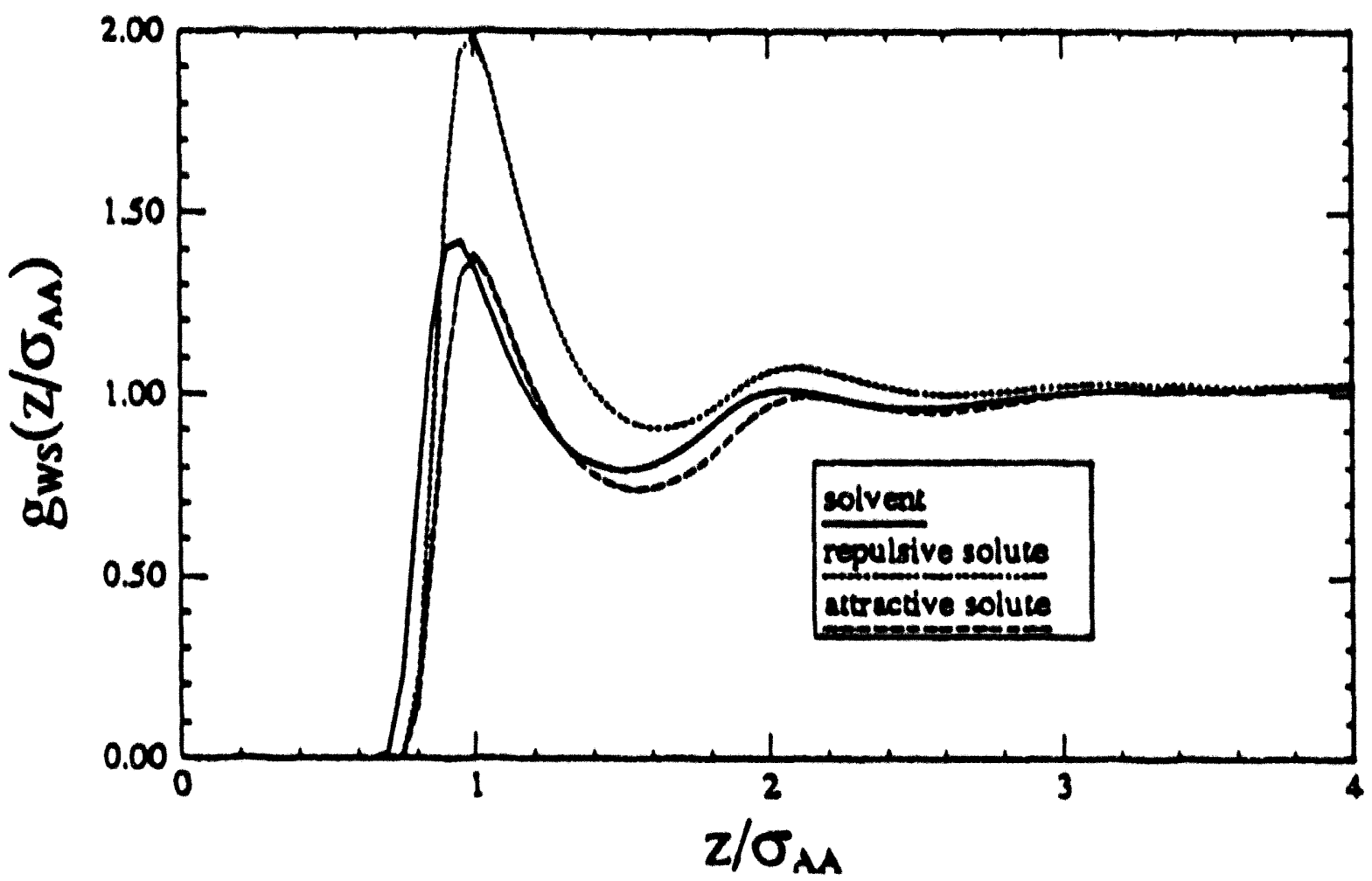

Figure 9.5 (b) Competitive adsorption between wall and repulsive or attractive solute. Wall-particle profiles 8ws are identified in the legend. $50 \% \mathrm{LJ}: 9-3$ wall. 


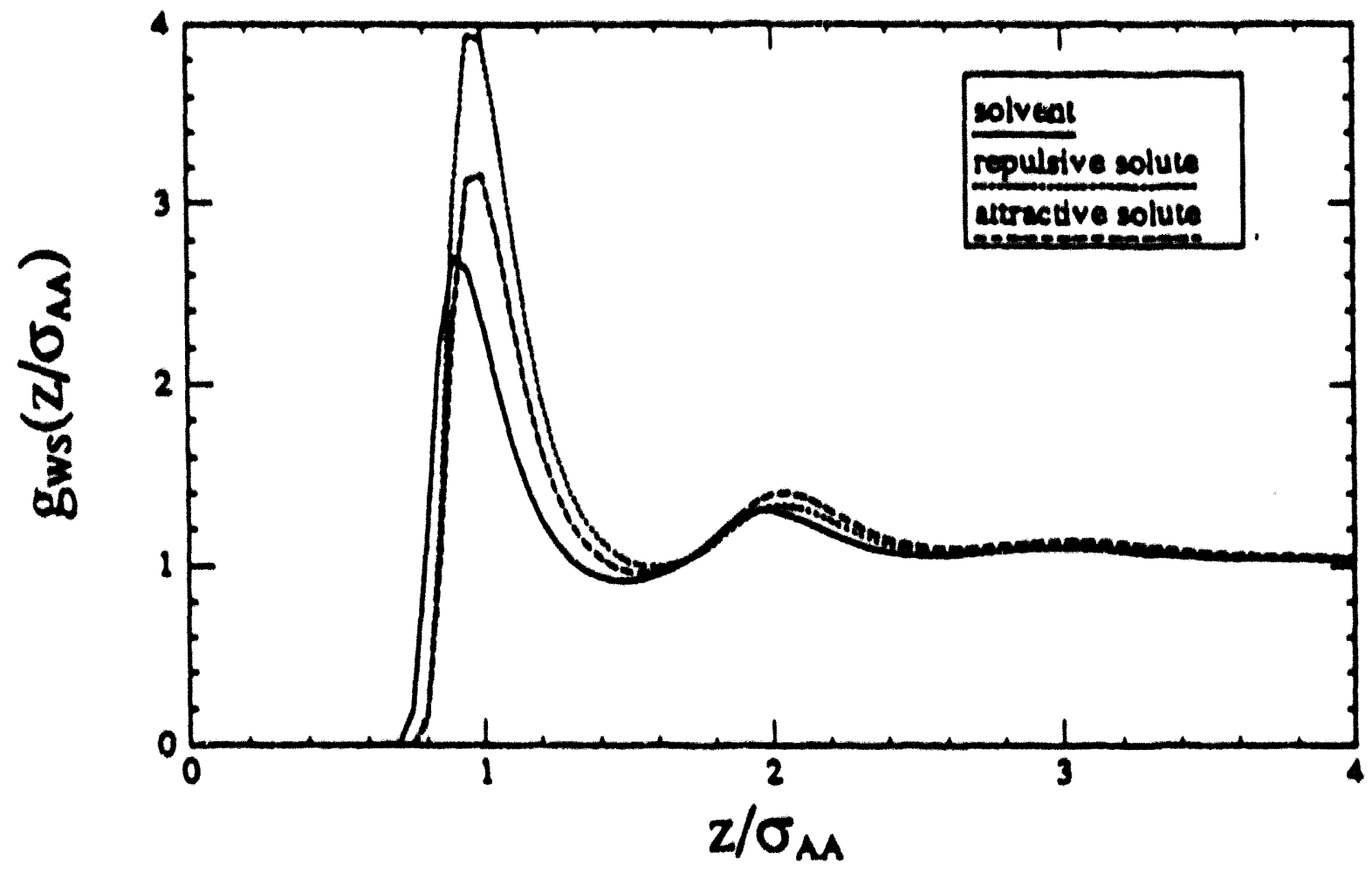

Figure 9.5 (c) Competitive adsorption between wall and repulsive or attractive solute. Wall-particle profiles $8 w s$ are identified in the legend. 75\% L:9.3 wall. 


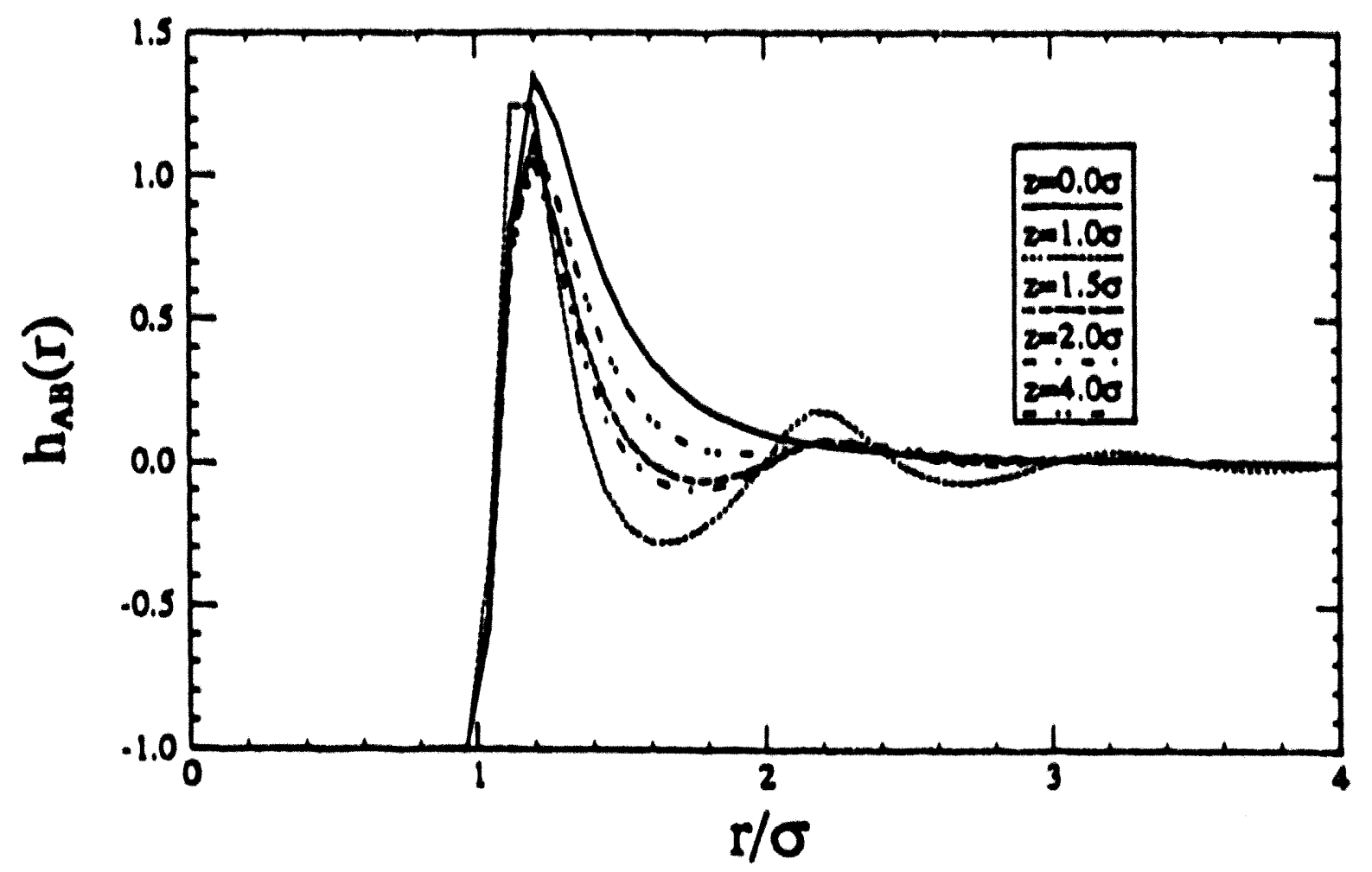

Figure 9.6 (a) Effect of distance from wall on radial distribution. Solutesolvent distribution $h_{A}$ 


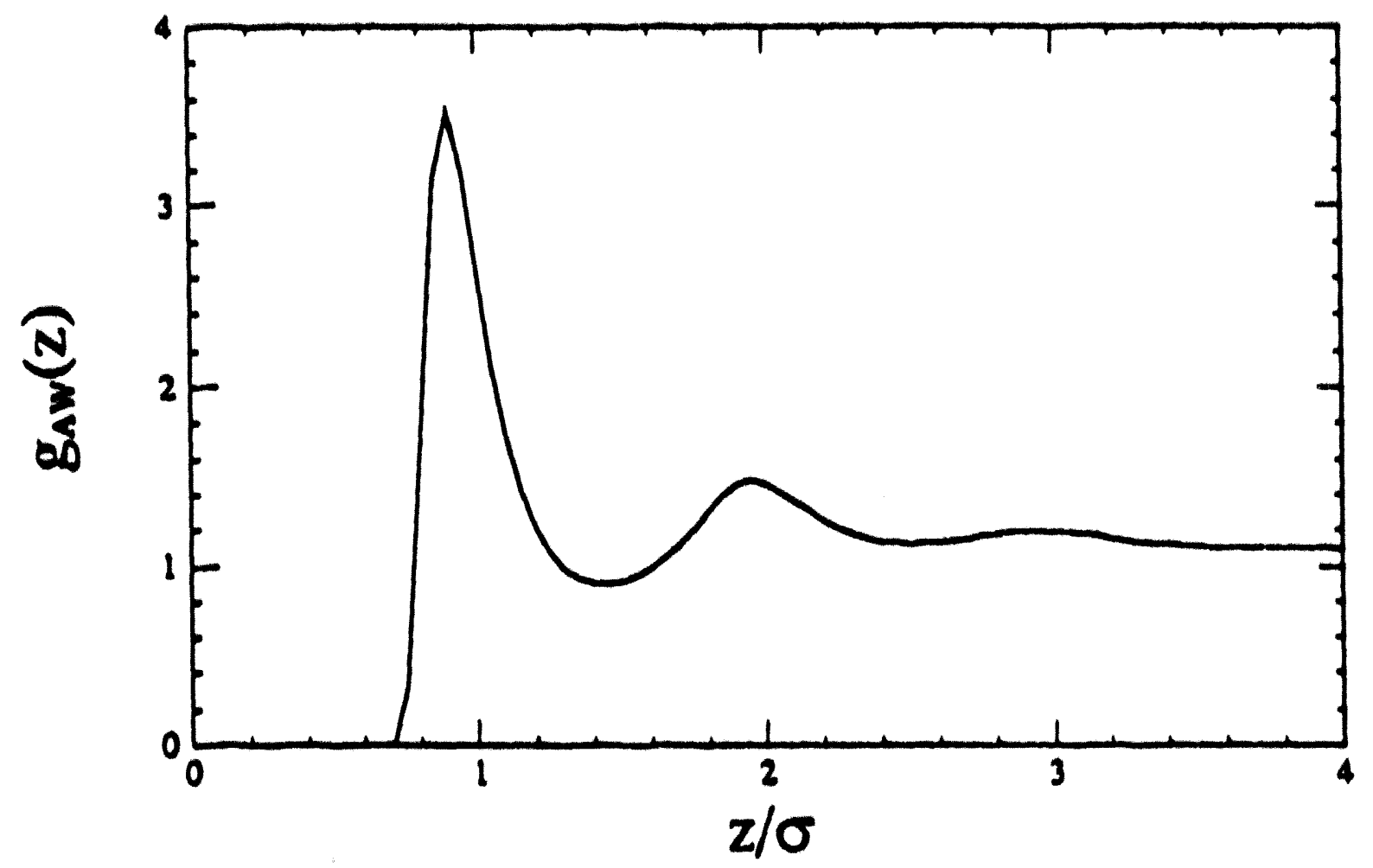

Figure 9.6 (b). Effect of distance from wall on radial distribution. Wall-particle profile saw. 

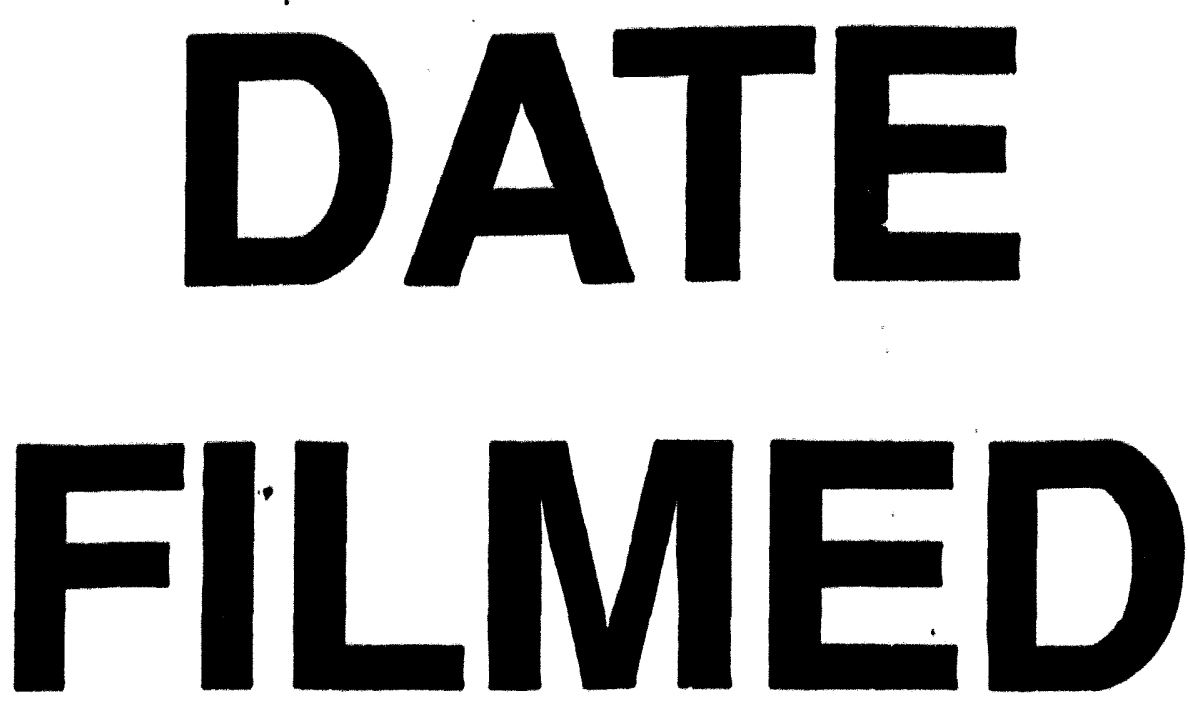

$12 / 27 / 93$
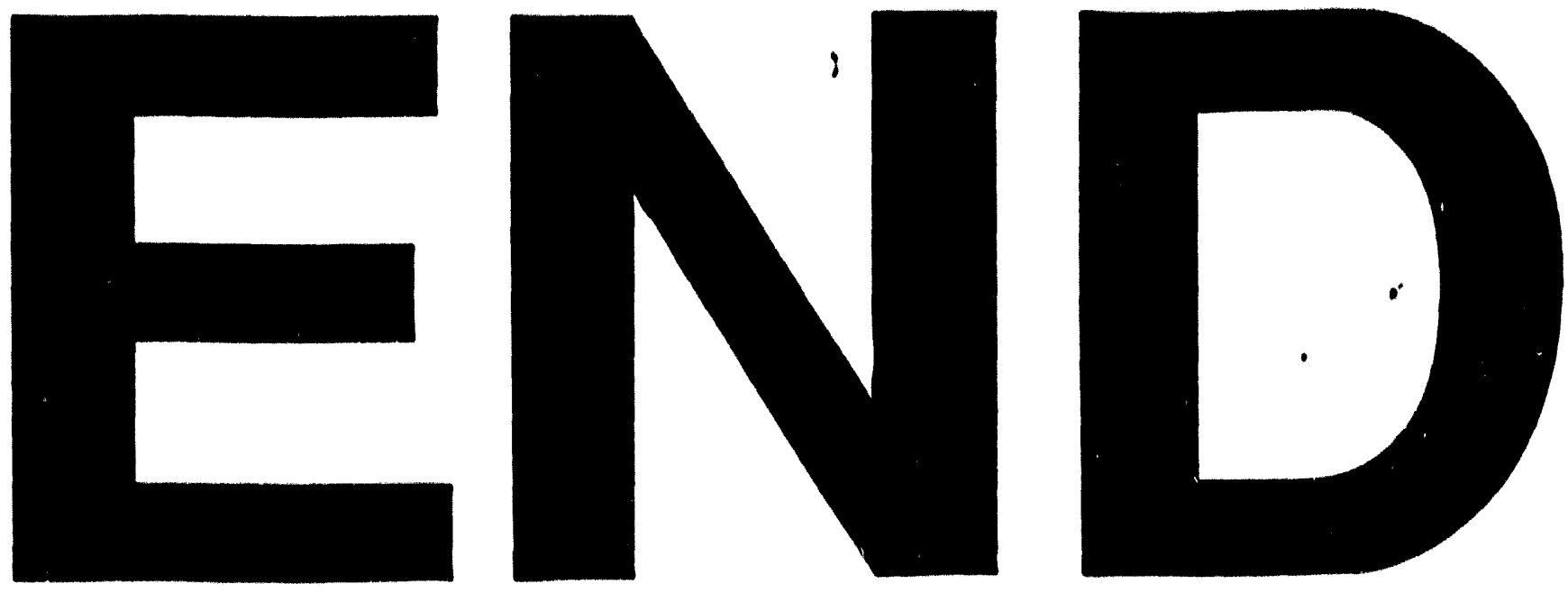